\title{
A Framework for Incorporating SPICE Models in Obreshkov based High-Order Circuit Simulation
}

\author{
by \\ Nikhil Bakshi
}

A thesis submitted to the Faculty of Graduate and Postdoctoral Affairs in partial fulfillment of the requirements for the degree of

Master of Applied Science

in

Electrical and Computer Engineering

Department of Electronics

Ottawa-Carleton Institute for Electrical and Computer Engineering

Carleton University

Ottawa, Ontario, Canada

(C) 2015

Nikhil Bakshi 


\section{Abstract}

This thesis describes a method to integrate SPICE device models in Obreshkov based circuit simulation.

Recently, a high-order A-stable and L-stable Obreshkov based method was proposed to simulate the transient response for general nonlinear circuits, which provides more than one order of magnitude speedup as compared to the traditional second order numerical integration methods used in the SPICE engine, without sacrificing the accuracy. This method requires calculation of higher order derivatives of Nodal equations, with orders higher than 2.

The purpose of this thesis is to develop a methodology to integrate industry standard SPICE device models in the Obreshkov based method. It describes the process to build a rooted tree from the source code of SPICE device models, and how to use this rooted tree to calculate high-order derivatives needed in the Obreshkov formula. This method was tested on the SPICE diode model, and the calculated values of the derivatives have been found to match the expected values up to 6 decimal places. 


\section{Acknowledgements}

I would like to extend my deepest gratitude to my co-supervisors, Prof. Emad Gad, Prof. Ram Achar and Prof. Michel Nakhla, who believed in me, provided the initial inspiration for this work, and gave critical guidance throughout the process. I would like to specially thank Prof. Emad Gad, for taking the time to look at my source code, for explaining me the concepts, and for helping me to break down the complex problem into manageable parts.

I would like to acknowledge and thank my colleagues, M. Farhan, D. Paul, A. Narayanan, G. Gosal, V. Gongal, B. Nouri, A. Charest, D. Trifkovic, N. Sovieko, A. Saini for the friendly discussions which often gave me a new perspective of looking at things. I would also like to express my gratitude to the professors, staff and students at the department of electronics for making my work an enjoyable experience.

I am grateful to my colleagues, Douglas Paul and Mina Farhan, who were patient enough to answer my questions and helped me navigate smoothly through the course of my research. I want to specially thank my manager, Micheal Ellis, at Canadian 
Bank Note Company Limited, for supporting me during my thesis, and for introducing me to good software development practices.

Finally, I thank my parents Sanjay and Shubhada Bakshi, for being patient with me, and keeping me motivated. I am grateful to you, for always leading by example, and for your unconditional love and support. 


\section{Contents}

$\begin{array}{lll}1 & \text { Introduction } & 1\end{array}$

1.1 General Description $\ldots \ldots \ldots \ldots \ldots$. . . . . . . . . . . . 1

1.2 Motivation . . . . . . . . . . . . . . . . . . . 2

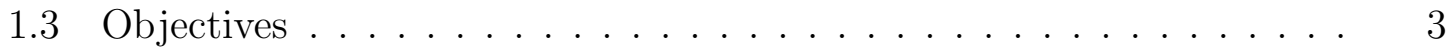

1.4 Contributions . . . . . . . . . . . . . . . . . 4

1.5 Thesis Organization $\ldots \ldots \ldots \ldots \ldots \ldots$

2 Review of Transient Simulation of Electrical Circuits 5

2.1 Problem Description . . . . . . . . . . . . . . . . 5

2.2 Accuracy of the method $\ldots \ldots \ldots \ldots$

2.3 Stability of the method . . . . . . . . . . . . 8

2.3.1 Stability of the Forward-Euler (FE) method . . . . . . . . . . 9

$2.3 .2 \quad$ Stability of the Backward Euler (BE) method . . . . . . . . . . 12

$2.3 .3 \quad$ Stability of the Trapezoidal Rule (TR) . . . . . . . . . . . . 13 
2.3 .4 Stability of Gear's method . . . . . . . . . . . . . . . . . 14

$2.4 \quad$ A-stability vs L-stability $\ldots \ldots \ldots \ldots$

2.5 Time-Domain Circuit Simulation . . . . . . . . . . . . . 17

2.6 Loading circuit element stamps . . . . . . . . . . . . . . . . 19

2.6 .1 Nonlinear elements . . . . . . . . . . . . . . . . . . . . . . . . 21

2.6 .2 Capacitive elements . . . . . . . . . . . . . . 22

2.6 .3 Resistive elements . . . . . . . . . . . . . . . . . . . 24

2.7 Accuracy vs Stability . . . . . . . . . . . . . . . . . . . 24

3 Review of Obreshkov based circuit simulation 26

3.1 Characteristics of the Obreshkov Formula $\ldots \ldots \ldots$. . . . . . . . . . 27

3.2 Obreshkov based Circuit Simulation . . . . . . . . . . . . . . 28

3.3 Factorization of the Jacobian Matrix . . . . . . . . . . . . . . . . 31

3.3.1 Structural properties of the Jacobian matrix . . . . . . . . . 31

3.3 .2 Reducing the size of the Jacobian matrix . . . . . . . . . . . . . 32

3.3 .3 Block Factorization of the Jacobian Matrix . . . . . . . . . . . . 33

3.4 Evaluating derivatives of nonlinear vector $\ldots \ldots \ldots$. . . . . . . 36

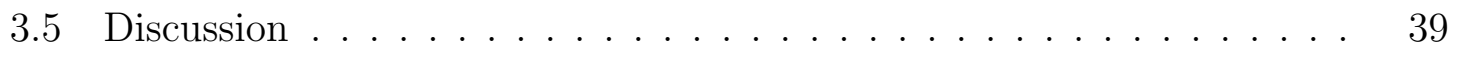

4 Structure of the SPICE device model source code 41

4.1 Loading a Device model in SPICE . . . . . . . . . . . . . . 42 
4.2 Data Structures to hold netlist data $\ldots \ldots \ldots \ldots \ldots \ldots$

4.2 .1 GENmodel . . . . . . . . . . . . . . . . . . . . . . . . . 47

4.2 .2 GENinstance . . . . . . . . . . . . . . . . . . . . 49

$4.2 .3 \quad$ The master data structure $\ldots \ldots \ldots \ldots$. . . . . . . . . . . . 50

4.2 .4 CKTcircuit structure _. . . . . . . . . . . . . 52

$4.3 \quad$ DEVload function . . . . . . . . . . . . . . . . . . . . . 53

4.3 .1 Nonlinear behaviour $\ldots \ldots \ldots \ldots \ldots$. . . . . . . . . 54

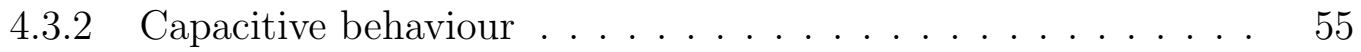

4.3 .3 Conductive behaviour . . . . . . . . . . . . . . 56

4.4 The SPICE diode model $\ldots \ldots \ldots \ldots$. . . . . . . . . . . . . . . 57

5 Generating the rooted tree from SPICE device model source code 62

5.1 The problem statement $\ldots \ldots \ldots \ldots \ldots \ldots$

5.2 Data Structure for the rooted tree $\ldots \ldots \ldots \ldots \ldots$. . . . . . . 64

5.3 Observations on the C-language code for SPICE device model . . . . 66

5.4 Implementation $\ldots \ldots \ldots \ldots \ldots \ldots \ldots \ldots \ldots \ldots \ldots \ldots$

$5.4 .1 \quad$ Generating the AST $\ldots \ldots \ldots \ldots \ldots \ldots \ldots$

5.4 .2 Converting the Clang AST to IVariableDefinition _. . . . 76

5.4 .3 Storing the internal IV ariableDefinition in memory . . . . . 80

5.4 .4 Converting IVariableDefinition to $i$ Expr . . . . . . . . . . 82

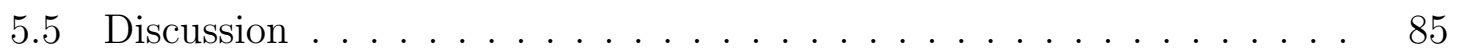


\begin{tabular}{lll}
\hline 6 & Numerical Results & 87 \\
\hline
\end{tabular}

6.1 Results . . . . . . . . . . . . . . . . . . . . . . . . 87

6.2 Extracting values of the constants required in the rooted tree . . . . 88

6.3 Evaluating derivatives of the rooted tree . . . . . . . . . . . . . 91

$\begin{array}{lll}7 & \text { Conclusions and Future Work } & 93\end{array}$

7.1 Conclusions $\ldots \ldots \ldots \ldots \ldots$. . . . . . . . . . . . . . . . . 93

7.2 Future Work . . . . . . . . . . . . . . . . . . . . . . 94

\begin{tabular}{|ll}
\hline Appendices & 96
\end{tabular}

\begin{tabular}{|ll}
\hline A Source code for CKT circuit struct & 97
\end{tabular}

\begin{tabular}{ll}
\hline S Source code of the DIOload function & 102
\end{tabular} 


\section{List of Figures}

2.1 Region of A-stability for FE (shaded area) . . . . . . . . . . . . 11

2.2 Region of A-stability for BE (shaded area) $\ldots \ldots \ldots \ldots$

2.3 Region of A-stability for TR (shaded area) . . . . . . . . . . . . . . . 14

2.5 Companion model for nonlinear current $\ldots \ldots \ldots$. . . . . . . 22

2.6 Companion model for capacitor . . . . . . . . . . . . 23

$4.1 \quad$ Data structures involved in loading SPICE device models . . . . . . 43

$4.2 \quad$ Inheritance hierarchy of GENmodel and GENinstance . . . . . . . 46

$4.3 \quad$ Linked List structure of Models and Instances for diodes in Listing (4.1) 47

4.4 SPICE Diode Model . . . . . . . . . . . . . . . . . . . . . . . . . 60

$5.1 \quad$ Tree for variable $e v d$. . . . . . . . . . . . . . . . . . . . . . 69

5.2 Tree for variable $v t e \ldots \ldots \ldots \ldots$. . . . . . . . . . . . . . . 69

5.3 Tree for variable $v t \ldots \ldots \ldots \ldots$. . . . . . . . . . . . . . . 69

5.4 Building the Tree for variable $v t \ldots \ldots \ldots \ldots$. . . . . . . 70 
$5.5 \quad$ Expanded Tree for $e v d \ldots \ldots \ldots \ldots \ldots$. . . . . . . . . . . . 71

5.6 Process of obtaining a rooted tree definition for variable, from the SPICE device model code . . . . . . . . . . . . . . . . . . . . . 72

5.7 Stages during compilation process . . . . . . . . . . . . . . . . 73

5.8 Inheritance Diagram for IVariableDefinition $\ldots \ldots \ldots \ldots$. . . . . . 77

6.1 Peak Rectifier Circuit . . . . . . . . . . . . . . . . . . . . 87

6.2 Comparison of Peak Rectifier output of Obreshkov method of order (5, 5) with HSPICE . . . . . . . . . . . . . . . . . . 91 


\section{List of Algorithms}

5.1 Building internal Tree representation . . . . . . . . . . . . 83

5.2 subExpandNode for ordinary Tree $:$ Node . . . . . . . . . . . . 84

5.3 subExpandNode for Tree : CondNode . . . . . . . . . . . . 85

5.4 Converting Tree to $i E x p r \ldots \ldots \ldots \ldots \ldots$ 


\section{List of Listings}

4.1 SPICE netlist for Bridge Rectifier . . . . . . . . . . . . . . . 45

4.2 Casting a derived-struct pointer to base-struct pointer . . . . . . . 46

4.3 Definition of device model $\ldots \ldots \ldots \ldots$

4.4 Definition of Diode model . . . . . . . . . . . . . . . . . . . . . . . 49

4.5 Definition of device instance $\ldots \ldots \ldots \ldots$. . . . . . . . . . . 50

$4.6 \quad S P I C E$ dev master data structure . . . . . . . . . . . . . . . . . . 51

$4.7 \quad$ IFdevice structure that defines the device in SPICEdev . . . . . . . . . 51

4.8 Loading of the SPICE diode model in DIOload function . . . . . . . 57

5.1 Moment function for mulTerm . . . . . . . . . . . . . . 66

5.2 Loading of the SPICE diode model in DIOload function . . . . . . . 68

$5.3 \quad$ Example C code to parse with Clang . . . . . . . . . . . . . . . . . . 74

5.4 Clang AST for C source code in Listing (5.3) . . . . . . . . . . . . 74

$5.5 \quad$ Loading of the SPICE diode model in DIOload function . . . . . . . . 79 
5.6 Tree Data Structure . . . . . . . . . . . . . . . . . . . . . . . . 83

6.1 Rooted Tree for nonlinear current of the diode . . . . . . . . . . . 89 


\section{Abbreviations}

ODE Ordinary Differential Equation.

NDAE Nonlinear Differential Algebraic Equation.

LTE Local Truncation Error.

NR Newton-Raphson iterative method.

MNA Modified Nodal Analysis.

TR Trapezoidal Rule integration method.

FE $\quad$ Forward Euler integration method.

BE Backward Euler integration method.

AST Abstract Syntax Tree.

DFS Depth First Search.

GDB GNU Debugger 


\title{
List of Symbols
}

\author{
$\alpha_{i}, \beta_{i} \quad$ Integration coefficients in modified Obreshkov formula. \\ $\alpha_{i, m} \quad$ Integration coefficients in Obreshkov formula. \\ $\mathbb{R}, \mathbb{C} \quad$ Real, Complex variable space. \\ $\boldsymbol{I}$ An Identity matrix. \\ $\boldsymbol{G} \quad$ A matrix that represents the memory-less elements in the MNA equations. \\ C A matrix that represents the memory elements in the MNA equations. \\ $\boldsymbol{f}(\boldsymbol{x}(t)) \quad$ A vector that contains the nonlinear currents flowing in nonlinear resistors and \\ nonlinear charges across nonlinear capacitors in the MNA equations. \\ $\boldsymbol{u}(t) \quad$ A vector that contains the values of the independent sources in a circuit at a \\ given time $t$. \\ $\boldsymbol{J} \quad$ The Jacobian matrix.


$\tilde{\boldsymbol{G}} \quad$ An augmented $\boldsymbol{G}$ Matrix that results from applying Obreshkov formula to MNA equations.

$\tilde{C} \quad$ An augmented $\boldsymbol{C}$ Matrix that results from applying Obreshkov formula to MNA equations.

$\tilde{\boldsymbol{\rho}} \quad$ A vector that contains the $h$-scaled high-order derivatives of circuit nonlinearities w.r.t MNA equations unknowns, and results from applying Obreshkov formula to the MNA equations.

$\tilde{\boldsymbol{u}}(t) \quad$ A vector that contains the derivatives of the independent sources in a circuit at a given time $t$.

$\tilde{\boldsymbol{J}} \quad$ Block Jacobian matrix.

$\boldsymbol{J}_{i, j} \quad$ The entry $(i, j)$ of matrix $\tilde{\boldsymbol{J}}$.

$\boldsymbol{R}_{u} \quad$ Matrix valued $u^{\text {th }}$ Taylor series coefficients of $\boldsymbol{J}_{0,0}$.

$\boldsymbol{x}_{n} \quad$ Approximation of $\boldsymbol{x}(t)$ at $t=t_{n}$.

$h \quad$ Current step size.

$h_{n} \quad$ Step size between $t_{n-1}$ and $t_{n}$. 


\section{Chapter 1}

\section{Introduction}

\subsection{General Description}

Transient Simulation of electronic circuits is an important process in the design automation as it is essential for the analysis and validation of electronic circuits. TimeDomain transient simulators use the process of solving Ordinary Differential Equations (ODEs) that numerically model a given circuit. The traditional SPICE-based simulators faced the limitation posed by the inherent conflict between the Order and the Stability of the methods used in solving these Differential Equations [1].

A new higher-order integration method was recently developed [2] which is based on Obreshkov Formula [3,4]. This new method does not suffer from the above con-

flict. It can be seen that this new method allows achieving the desired property of A-stability (or L-stability) with higher-order approximation of the solution of Dif- 
ferential Equations [5,6]. As a result of this, the transient simulators based on the Obreshkov method run much faster than the classical low-order methods that are used in the traditional SPICE-based simulators such as Gears Method or Trapezoidal Rule. Recent advances as reported in [7 [9] also consistently demonstrate that this new method improves speed-up for the time-domain transient simulation.

\subsection{Motivation}

The Obreshkov-based method relies on computing the high-order derivatives of the circuit variables with respect to time. In order to perform this computation, the Obreshkov method requires computing the high-order derivatives of the nonlinear device responses. Unfortunately, the popular device models developed for the classical SPICE engines are not adequate for computing the high-order derivatives. These models have been developed for the low-order methods and can only provide the firstorder derivative of the device response. Thus, if a developer of a circuit simulator is interested in creating a new circuit simulator based on the Obreshkov method, he will be left with only two choices. The first choice is to rewrite the code of the existing SPICE models with the goal of computing the high-order derivatives. The other choice is to find a way to use the existing device model code, without modification, to extract the high-order derivatives.

Given that the existing device model code is a result of many years of accumulated 
experience, the first choice appeared to be very costly, since it requires understanding of many lines of code, which in many situations do not have adequate documentation. The goal of this thesis is to explore the second choice as will be explained in the next section.

\section{$1.3 \quad$ Objectives}

The objective of this thesis is to develop a framework to automate the process of incorporating the existing device models in the Obreshkov method. The approach followed is based on generating a rooted-tree structure that captures the device nonlinearity, and use that structure to compute the high-order derivatives. The rooted tree will be generated from the SPICE device models (written in C language) using the LLVM-Clang tool to parse the $\mathrm{C}$ source code. In order to achieve this, the thesis will dwell upon the methodology used by the open source SPICE implementation (known as ngSPICE) to incorporate the device models into the core of the SPICE engine. The idea here is to discern the pattern that is used by the classical SPICE engine to access the device information, with the objective of adapting this pattern towards the goal of computing the high-order derivatives. 


\subsection{Contributions}

The contributions of this thesis are as follows. This thesis develops a framework to integrate the SPICE device models in Obreshkov based high-order circuit simulation. By systematically exploiting the structure of the $\mathrm{C}$ language based source code for SPICE device models, we identify relevant sections in the source code which model the behaviour and characteristics of the devices, and then we develop a technique to parse the $\mathrm{C}$ source code, to extract the relevant information, and also to generate the rooted tree for modeling the nonlinear behaviour of the device.

\subsection{Thesis Organization}

This thesis is organized among six chapters. Chapter 2 reviews common integration methods. Chapter 3 reviews the higher-order method based on Obreshkov Formula. Chapter 4 presents the structure of the $\mathrm{C}$ language based source code for the SPICE device models. Chapter 5 presents the technique of parsing the relevant details from the SPICE device model $\mathrm{C}$ source code, and generating the rooted tree for the nonlinear behaviour of the device. The last chapter presents the results of integrating the SPICE diode models in Obreshkov based simulation. 


\section{Chapter 2}

\section{Review of Transient Simulation of Electri-}

\section{cal Circuits}

This chapter provides an overview of the transient domain simulation methods used in the SPICE-type simulators. We will look at the different numerical integration methods used to solve nonlinear differential equations, and compare the different methods based on their accuracy and stability characteristics.

\subsection{Problem Description}

The behaviour of electronic circuits can be modeled by the following system of differential equations:

$$
\frac{d \boldsymbol{x}(t)}{d t}=\boldsymbol{f}(x, t)
$$


This is a first-order nonlinear system of differential equations, where $\boldsymbol{x}(t) \in \mathbb{R}^{N}$, and $\boldsymbol{f}(x, t)$ is a nonlinear mapping. Solving the above system of equations amounts to finding $\boldsymbol{x}(t)$ that satisfies the above equation. Instead of analytically finding the function $\boldsymbol{x}(t)$, the numerical integration methods used to solve the above equation, approximate the values of $\boldsymbol{x}(t)$ at a set of discrete time points $t_{0}, t_{1}, t_{2}, \ldots$ over a range of time. The corresponding values of $\boldsymbol{x}(t)$ at these time points are denoted by $\boldsymbol{x}\left(t_{0}\right), \boldsymbol{x}\left(t_{1}\right), \boldsymbol{x}\left(t_{2}\right), \ldots$ etc. The numerical integration methods are characterized by their accuracy and stability. We discuss these concepts next.

\section{$2.2 \quad$ Accuracy of the method}

A numerical integration method is said to be of order $p$, if the following criteria is met:

$$
\boldsymbol{x}_{n}=\boldsymbol{x}\left(t_{n}\right)+\left.C h_{n}^{p+1} \frac{d^{p+1}}{d t^{p+1}} \boldsymbol{x}(t)\right|_{t=t_{n}}+O\left(h_{n}^{p+2}\right)
$$

where $h_{n}=t_{n}-t_{n-1}$, is the time step, $C$ is the error constant of the method [10], whose value indicates the accuracy of the method being used, and $O\left(h^{v}\right)$ are all the terms in the infinite series whose power of $h$ is greater than or equal to $v$. In order to approximate $\boldsymbol{x}_{n}$, we can choose to ignore terms in the infinite series whose powers of $h$ are higher than a certain $v$. If we ignore the above term $O\left(h^{v}\right)$, then the local 
truncation error of the above approximation can be written as:

$$
L T E=\left.C h_{n}^{p+1} \frac{d^{p+1}}{d t^{p+1}} \boldsymbol{x}(t)\right|_{t=t_{n}}
$$

We can see that in the above equation, the power of $h^{n}$ is same as the power of $\frac{d x}{d t}$. Also, for a small step size $h_{n}$, the local truncation error is proportional to $h_{n}^{p+1}$, which depends on the order $p$. Thus, a higher-order integration method will allow us to use a large step size, and will give the same amount of accuracy as compared to a lower order method with a smaller time step size. It is favourable for us to use larger step size in time domain simulation, because we will have fewer time points to approximate $\boldsymbol{x}(t)$ for analyzing the simulation. Hence it is preferable for us to use high-order integration methods.

However, it is important that the numerical integration method of any order that is used, should be stable, i.e. its solution must be bounded over time. The next section shows how to evaluate the stability of the integration method used. 


\subsection{Stability of the method}

To determine the stability of a numerical integration method, we consider the following differential equation:

$$
\begin{gathered}
\frac{d x}{d t}=\lambda x(t) \\
x(0)=x_{0}
\end{gathered}
$$

where lambda is a complex number [11].

The above differential equation is said to be stable if the following condition is satisfied:

$$
x_{n}<x_{m} \text { for } n>m
$$

which means that as the time progresses, the successive approximations of $\boldsymbol{x}(t)$ grow smaller, and the solution is bounded.

Whether the differential equation in 2.4 is stable or not, is determined by the value of $\lambda$. Not all differential equations of the type (2.4) have a stable bounded solution $\boldsymbol{x}(t)$. Hence, the stability domain of the equation 2.4 is defined by all the values of $\lambda$, for which equation (2.4) has a stable solution $\boldsymbol{x}(t)$.

Using Laplace transforms on equation (2.4), we can say that for all lambda that belongs to the complex left half plane $\left(\lambda \in \mathbb{C}^{-}\right)$, the solution $\boldsymbol{x}(t)$ will be a monoton- 
ically decreasing function of time.

Hence it is reasonable to assume that the numerical approximation of $\boldsymbol{x}(t)$, obtained by the numerical integration method, should also be a monotonically decreasing function over time, for $\lambda$ in the left half plane. An integration method that yields monotonically decreasing solutions, for $\lambda$ in the left half plane, is known as A-stable. A-stability is thus a very desirable property to have for any numerical integration method. In the next section, we will evaluate different numerical integration methods for A-stability.

\subsubsection{Stability of the Forward-Euler (FE) method}

The Forward Euler (FE) integration method is defined as

$$
x_{n+1}=x_{n}+h x_{n}^{\prime}
$$

This means that given a time point $t_{n}$, whose approximation $x_{n}$, and first-order derivative $x_{n}^{\prime}$ at $t=t_{n}$ is known, the approximation $x_{n+1}$ at the next time point $t_{n+1}$, is calculated using the known values $x_{n}$, and derivative $x_{n}^{\prime}$ at $t_{n}$, where $h$ is the time step $h=t_{n+1}-t_{n}$. This is an integration method of order 1 .

Substituting (2.7) in the differential equation (2.4), we get the following recursive 
formula:

$$
x_{n+1}=(1+h \lambda) x_{n}
$$

This can be further simplified to:

$$
x_{n+1}=(1+h \lambda)^{(n+1)} x_{0}
$$

For the above equation to satisfy the stability criteria $(2.6)$, we must have

$$
|1+h \lambda|<1
$$

Since $\lambda$ is a complex number, we can write:

$$
|1+x+i y|<1 \text { or }\left[(1+x)^{2}+z^{2}\right]<1
$$

This is the equation of a circle in the complex plane, with centre at $(-1,0)$ and radius of 1 . This is shown in Figure 2.1.

Thus we can see that the FE method gives a exponentially decreasing solution, only if the values of $h \lambda$ lie in the above circle in the complex plane. An integration method is A-stable, only if it provides bounded solutions for all values of lambda in the negative half complex plane. Thus Forward Euler is not A-stable method. 


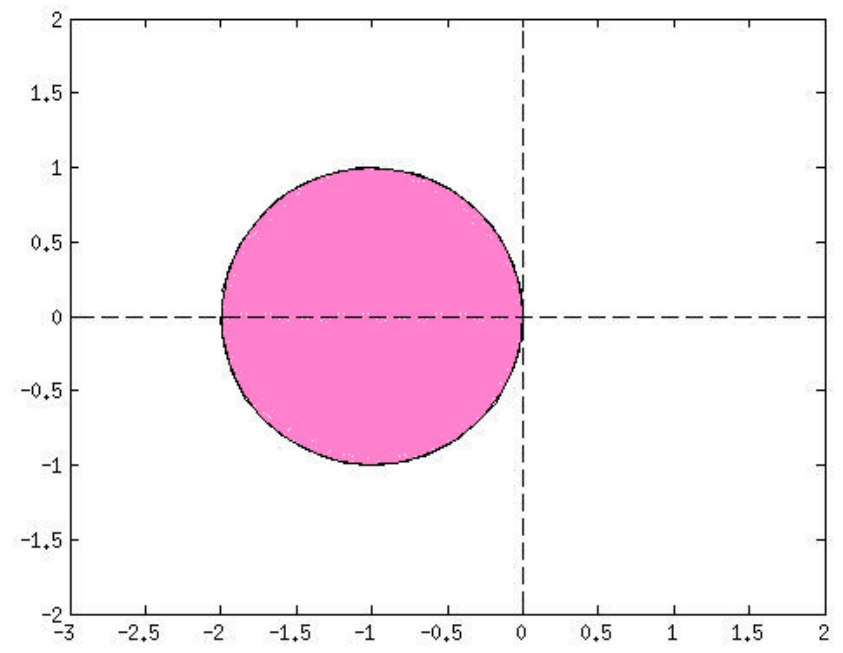

Figure 2.1: Region of A-stability for FE (shaded area)

The actual solution to the differential equation in $(2.4)$ is:

$$
x\left(t_{n+1}\right)=e^{\lambda t_{n+1}} x(0)
$$

which we can see is exponentially decreasing for all values of lambda in the left half complex plane.

Even if we were to use FE, then on a differential equation with a very high lambda, we would have to use a very small step size $h$, for $h \lambda$ to fall in the above circle. Thus the FE integration method is not very useful in practical applications. 


\subsubsection{Stability of the Backward Euler (BE) method}

The Backward Euler (BE) method is defined as:

$$
x_{n+1}=x_{n}+h x_{n+1}^{\prime}
$$

Equation (2.13) implies that the approximation at the next time point $t_{n+1}$, is determined by using the approximation $x_{n}$ at the current time point $t_{n}$, and the derivative $x_{n+1}^{\prime}$ at the next time point $t_{n+1}$. This is an integration method of order 1 . Using this in the differential equation (2.4), we get the recursive formula:

$$
x_{n+1}=(1-h \lambda)^{-1} x_{n}
$$

Thus the stability domain of BE comes out to be:

$$
|1-h \lambda|^{-1}<1
$$

This region is shown graphically by the Figure 2.2. As can be seen in Figure 2.2. the BE method gives a decreasing solution in the entire left half complex plane, irrespective of the value of step size $h$. Thus BE method is A-stable. 


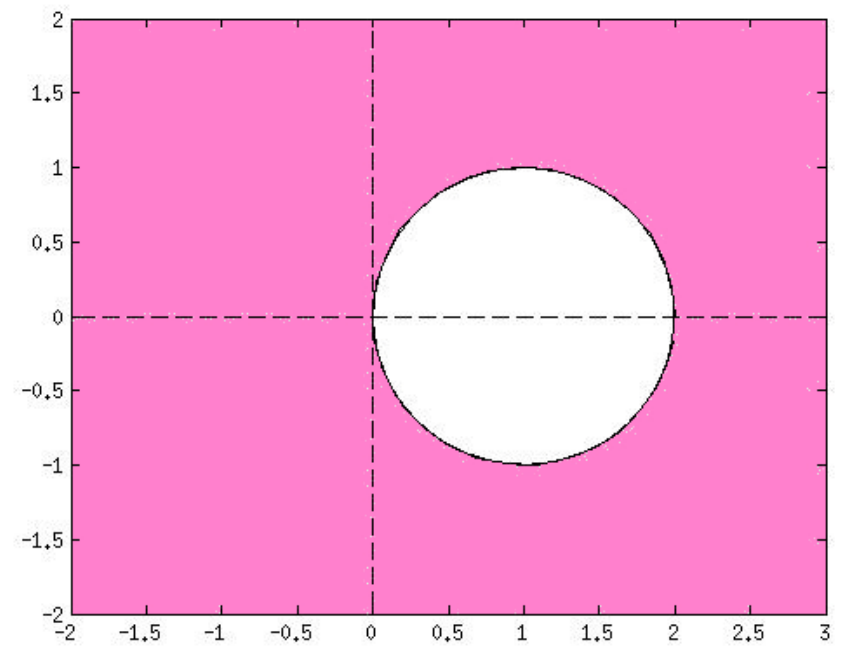

Figure 2.2: Region of A-stability for BE (shaded area)

\subsubsection{Stability of the Trapezoidal Rule (TR)}

Trapezoidal Rule (TR) is defined as:

$$
x_{n+1}=x_{n}+h \frac{\left(x_{n+1}^{\prime}+x_{n}^{\prime}\right)}{2}
$$

This is an integration method of order 2. Substituting this in the differential equation (2.4) gives us:

$$
x_{n+1}=\frac{\left(1+\frac{h \lambda}{2}\right)}{\left(1-\frac{h \lambda}{2}\right)} x_{n}
$$

The stability domain of the Trapezoidal rule is shown in Figure 2.3. Thus TR gives decaying solutions for all values of lambda in the complex left half plane, irrespective 


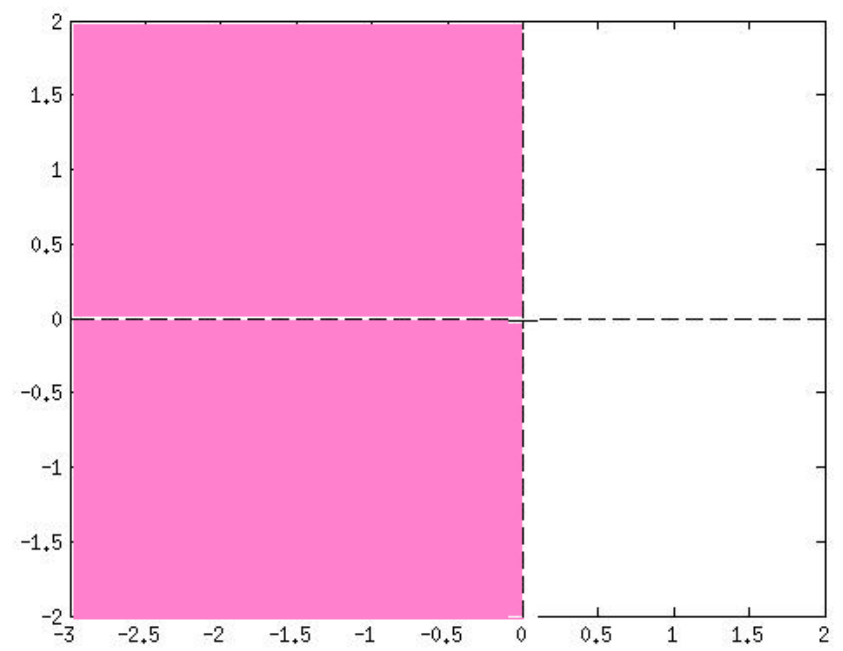

Figure 2.3: Region of A-stability for TR (shaded area)

of the value of h. Thus TR is A-stable.

\subsubsection{Stability of Gear's method}

Gear's method is a family of methods which are defined as:

$$
\sum_{k=0}^{s} \alpha_{k} x_{(n+k)}=h \beta x_{n+s}^{\prime}
$$

where $\alpha_{k}$ and $\beta$ are coefficients which are chosen to make the method achieve an order $s$. To obtain the coefficients $\alpha_{k}$ and beta in equation (2.18), we substitute $\boldsymbol{x}\left(t_{n+s-k}\right)$, $\left.\frac{d x}{d t}\right|_{t=t_{n+s}}$ for $x_{n+s-k}, k=0,1,2, \ldots, s$, and $x_{n+s}^{\prime}$ in 2.18, expand each term in it's Taylor Series, and equate to zero, all the terms up to the $s^{t h}$-derivative [12]. For example, 


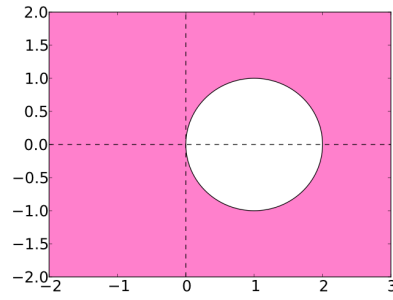

(a) Order 1

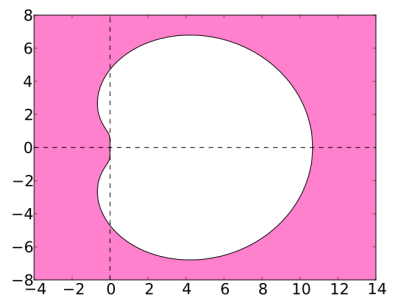

(d) Order 4

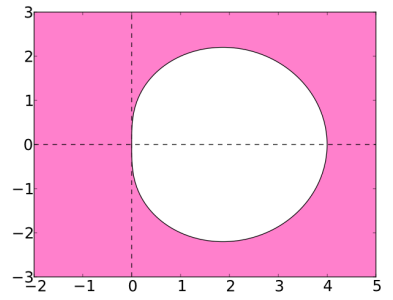

(b) Order 2

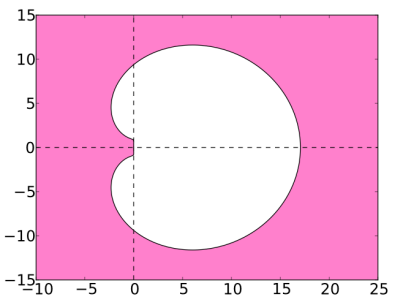

(e) Order 5

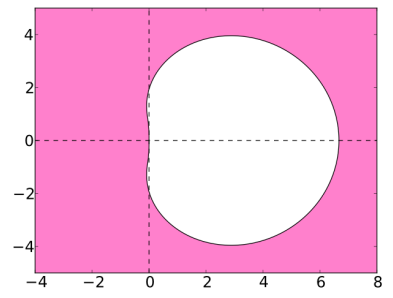

(c) Order 3

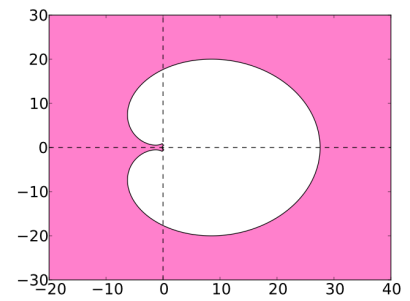

(f) Order 6

Figure 2.4: Region of A-stability for Gear's method from order 1 to 6 (shaded area)

when we set $s=2$, the Gear's method for order 2 can be described as:

$$
x_{n+2}-\frac{4}{3} x_{n+1}+\frac{1}{3} x_{n}=\frac{2}{3} h x_{n+2}^{\prime}
$$

Figure 2.4 shows the stability regions of the Gear's method for orders s $=1$ to 6 . It must be noted that only Gear's method of order 1 and 2 are A-stable. Beyond the second order, the stability region of Gear's method does not cover the entire left half of the complex plane. This is an indication that even though higher-order methods are more accurate, they will not necessarily be A-stable. 


\section{$2.4 \quad$ A-stability vs L-stability}

In the previous section (2.3), we discussed that if we are to chose an integration method to solve a system of differential equations, that integration method must be A-stable. However, A-stability alone is not enough to pick a numerical integration method. The concept of L-stability is equally important [13], and can be defined as:

$$
\lim _{|\lambda| \rightarrow \infty}\left|\frac{x_{n+1}}{x_{n}}\right|=0
$$

This means that as lambda goes far left to infinity in the left half plane, the successive approximations must die to zero. This makes physical sense, because the exact solution of the DE (2.4), given in 2.12), decays to 0 very quickly, for a highly negative value of $\lambda$.

A system of differential equations is said to be stiff, if the eigenvalues of the system are deep in the left-half of the complex plane. The exact solution of such a system of equations dies out to zero quickly, just like it does for a single differential equation according to 2.20 .

L-stable integration methods give approximations that die out to 0 very quickly, when $\lambda$ is high [14]. Therefore, for a system of stiff differential equations, it is im-

portant that the integration method used to solve the system is L-stable. From the 
recursive formula for $\mathrm{BE}$ in (2.14), we find that

$$
\begin{aligned}
& \frac{x_{n+1}}{x_{n}}=\frac{1}{1-h \lambda} \\
& \lim _{|\lambda| \rightarrow \infty}\left|\frac{x_{n+1}}{x_{n}}\right|=0
\end{aligned}
$$

Thus BE is L-stable. However, the recursive formula for TR in (2.17), gives:

$$
\begin{gathered}
\frac{x_{n+1}}{x_{n}}=\frac{\left(\frac{2}{h \lambda}+1\right)}{\left(\frac{2}{h \lambda}-1\right)} \\
\lim _{|\lambda| \rightarrow \infty}\left|\frac{x_{n+1}}{x_{n}}\right|=1
\end{gathered}
$$

This means that TR is not L-stable. So, even if the eigenvalues of the system of equations is deep in the left half plane, TR approximations will not decay to 0 as quickly as they should. So TR is not L-stable, and is not the best integration method to use if the system uses stiff differential equations.

\subsection{Time-Domain Circuit Simulation}

General electronic circuits contain several nonlinear elements like diode, transistors, etc. Using the Modified Nodal Analysis (MNA) method on such circuits, gives us a 
Nonlinear Differential Algebraic Equation (NDAE) of the form [15]:

$$
\mathbf{G} \mathbf{x}(t)+\mathbf{C} \frac{d \mathbf{x}}{d t}+\mathbf{f}(\mathbf{x}(t))=\mathbf{u}(t)
$$

where $\mathbf{G}$ and $\mathbf{C}$ are $\mathbb{R}^{N \times N}$ matrices that characterize the memoryless and energystoring elements of the circuit, $\mathbf{u}(t) \in \mathbb{R}^{N}$ is a vector representing the independent inputs to the circuit, $\mathbf{x}(t) \in \mathbb{R}^{N}$ is a vector containing the nodal voltages, and the currents of the inductors and voltage sources, $\mathbf{f}(\mathbf{x}(t))$ is the vector of nonlinear currents of the nonlinear elements in the circuit, $N$ is the total size of the MNA formulation. Solving this differential equation amounts to finding $\boldsymbol{x}(t)$ that satisfies the above differential equation. This equation can be solved by using one of the numerical integration methods discussed above.

For example, if we use the A-stable Backward Euler method to solve the equation (2.25), then substituting the BE formula 2.13) in equation (2.25), we get:

$$
\left(\mathbf{C}+h \mathbf{G} \mathbf{x}_{n+1}\right)+h \mathbf{f}\left(\mathbf{x}_{n+1}\right)=h \mathbf{u}\left(t_{n+1}\right)+\mathbf{C}\left(\mathbf{x}_{n}\right)
$$

The above system of equations is nonlinear and can be solved using Newton method, where we start with an initial guess of $\boldsymbol{x}_{0}^{n+1}$, and iteratively update it using a correction of:

$$
\Delta \mathbf{x}=\left.\left.\mathbf{J}^{-1}\right|_{x=x_{n+1}^{(0)}} \boldsymbol{\Phi}\left(\mathbf{x}_{n+1}\right)\right|_{x_{n+1}=x_{n+1}^{(0)}}
$$


where $\boldsymbol{J} \in \mathbb{R}^{N \times N}$ is the Jacobian matrix given by:

$$
\mathbf{J}=\mathbf{C}+h \mathbf{G}+h \frac{\partial \mathbf{f}}{\partial \mathbf{x}}
$$

and $\boldsymbol{\Phi}$ is the error in satisfying the system of equations given by:

$$
\boldsymbol{\Phi}\left(\mathbf{x}_{n+1}\right)=(\mathbf{C}+h \mathbf{G}) \mathbf{x}_{n+1}+h \mathbf{f}\left(\mathbf{x}_{n+1}\right)-\mathbf{C} \mathbf{x}_{n}-h \mathbf{u}\left(t_{n+1}\right)
$$

The Newton Method usually converges in a small number of iterations, provided

a good initial guess $\boldsymbol{x}_{n+1}^{0}$ is chosen. A good guess for $\boldsymbol{x}_{n+1}^{0}$, would be the solution of the previous time point $\boldsymbol{x}_{n}$. The system of equations in 2.27 can be solved with LU factorization.

\subsection{Loading circuit element stamps}

The modus operandi of circuit simulators like SPICE is to read a netlist of circuit elements and construct from it the Jacobian matrix $\boldsymbol{J}$ and the RHS vector $\boldsymbol{\phi}$ of equation 2.27. This is done by stamping the $\boldsymbol{J}$ and $\boldsymbol{\phi}$ matrices by the contribution of each element in the circuit. The job of the SPICE device models is to stamp the $\boldsymbol{J}$ and $\phi$ matrices directly, with the contribution of that element, given how the element is connected in the circuit, and it's parameters. 
The MNA equation 2.25 governing the behaviour of circuits is a nonlinear differential Algebraic equation. However, the Newton iteration equation (2.27) is a linearized algebraic form of equation (2.25). So nonlinear elements like diodes and transistors, will have to be linearized, before populating the $\boldsymbol{J}$ and $\boldsymbol{\phi}$ matrices with their contribution. Also, memory elements like capacitors and inductors are governed by differential equations, and their response will have to be expressed as algebraic equations using numerical integration methods. In SPICE, the most common numerical integration methods used are Trapezoidal Rule or Gear's Method [16]. The stamps that are thus developed for nonlinear and memory elements are called companion models. These companion models are approximations of the behaviour of the nonlinear device or memory device using linear memoryless devices like resistors, voltage and current sources. SPICE device models use these companion models to populate the $\boldsymbol{J}$ and $\boldsymbol{\phi}$ matrices.

In this section, we will understand the stamps for three main type of circuit elements, namely memoryless, memory and nonlinear elements, used in SPICE like simulators. The knowledge of device stamps developed in this section, will help us in section (4.4), where we will use these SPICE device stamps to extract information required for the Obreshkov based integration method, and drop the data related to the SPICE integration methods. 


\subsubsection{Nonlinear elements}

In this section, we will discuss the companion model for nonlinear elements used in SPICE like simulators. Let us begin by developing a companion model for a diode. The $i-v$ characteristics for a diode are given by the diode equation [17] as follows:

$$
i=I_{s}\left(e^{\frac{v}{n \times V_{T}}}-1\right)
$$

where $v$ is the voltage across the diode, and $i$ is the current through the diode. $I_{s}$ is the saturation current, $V_{T}$ is the threshold voltage, and $n$ is the idealization constant of the diode.

If we are given a point $\left(v_{n}, i_{n}\right)$ which satisfies this equation, then the Newton Method develops a linear approximation for $i$ in equation 2.30 by using the Taylor series approximation of $i$ upto the first derivative as follows:

$$
i=i_{n}+\left.\frac{d i}{d v}\right|_{v=v_{n}}\left(v-v_{n}\right)
$$

Here the known quantities are $v_{n}, i_{n}$ and $\left.\frac{d i}{d v}\right|_{v=v_{n}}$. If we denote $v_{n}$ by $v_{d}, i_{n}$ by $i_{d}$ and $\left.\frac{d i}{d v}\right|_{v=v_{n}}$ by $g d$, then equation 2.31 can be written as:

$$
i=\left(g_{d}\right) v+\left(c_{d}-g_{d} v_{d}\right)
$$


This can be represented by the circuit in Figure 2.5.

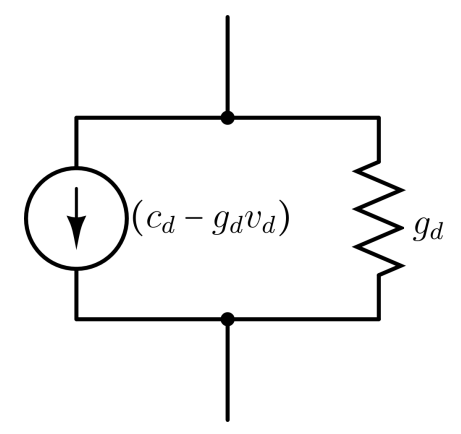

Figure 2.5: Companion model for nonlinear current

This gives rise to the following system of equations:

$$
\left[\begin{array}{cc}
g_{d} & -g_{d} \\
-g_{d} & g_{d}
\end{array}\right]\left[\begin{array}{l}
v_{p} \\
v_{n}
\end{array}\right]=\left[\begin{array}{l}
-\left(c_{d}-g_{d} v_{d}\right) \\
+\left(c_{d}-g_{d} v_{d}\right)
\end{array}\right]
$$

\subsubsection{Capacitive elements}

Capacitors give rise to differential equations. As discussed in section 2.1), we have to use numerical integration methods to convert the differential equations to algebraic equations. The behaviour of the capacitor is defined by the equation

$$
i=C \frac{d v}{d t}
$$


where $i, v$ are the current through, and voltage across the capacitor respectively. Applying say the Trapezoidal Rule equation (2.16) to equation (2.34), we get:

$$
\begin{array}{r}
v_{n+1}=v_{n}+\frac{h}{2 C}\left[i_{n+1}+i_{n}\right] \\
i_{n+1}=\left(\frac{h}{2 C}\right) v_{n+1}+\left[i_{n}-\frac{2 C}{h} v_{n}\right]
\end{array}
$$

where $i_{n}, v_{n}$ are the current and voltage at the present time point, and $i_{n+1}, v_{n+1}$ are the current and voltage at next time point.

The equation (2.36) gives the companion circuit of the capacitor, shown in Figure 2.6.

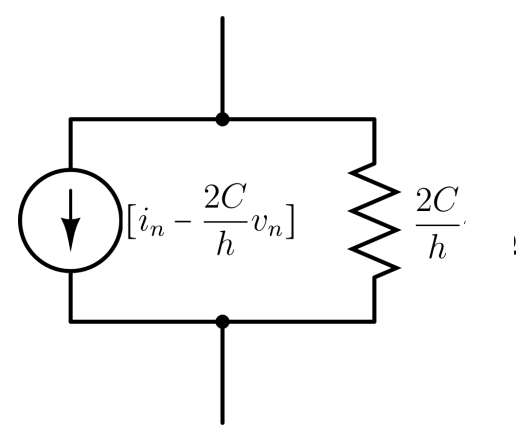

Figure 2.6: Companion model for capacitor

As can be seen from Figure 2.6, the $g_{e q}$ and $c_{e q}$ variables depend on the integration method being used. For a different integration method like the Gear's method, we will have a similar structure for the companion model of a capacitor, consisting of a resistor and current source, but the values of $g_{e q}$ and $c_{e q}$ will be different. 


\subsubsection{Resistive elements}

Resistive elements are loaded in the $\boldsymbol{J}$ and $\boldsymbol{\phi}$ matrices using the MNA resistor stamp 16, 18, which is as follows:

$$
\left[\begin{array}{cc}
G & -G \\
-G & G
\end{array}\right]\left[\begin{array}{l}
v_{\text {node } 1} \\
v_{\text {node } 2}
\end{array}\right]=\left[\begin{array}{l}
0 \\
0
\end{array}\right]
$$

where $\mathrm{G}$ is the conductance $\left(G=\frac{1}{R}\right), v_{\text {node } 1}, v_{\text {node2 } 2}$ are the terminal nodes of the resistor.

\subsection{Accuracy vs Stability}

As discussed before, the order of a numerical integration method, is a measure of the accuracy of the method. It indicates the amount of Local Truncation Error that the integration method incurs. A higher order method, usually has less error, and is more accurate, which allows us to use a larger step size. On the other hand, the stability of the method indicates whether the approximate solution to the DE is stable or not. For stable DE, that have stable exact solutions, we must use A-stable or Lstable integration methods [14], so that our approximation to the exact solution is also stable, as required. As seen in the case of Gear's method, when the order of the integration method is increased, the method is not A-stable. Thus, we can see 
that there is a conflict between order and stability of an integration method, as we start using higher-order integration methods. Recent work on the Obreshkov based integration method $[2]$ has found that we can ease the inherent conflict between order and stability, if we adopt higher order derivatives in the integration method in a prescribed order. This will be discussed in the next chapter. 


\section{Chapter 3}

\section{Review of Obreshkov based circuit simula-}

\section{tion}

Recent work on the Obreshkov Formula [2] has shown how higher order integration formulae can be used to solve system of Differential Equations, without suffering from the conflict between Order and Stability of the method. The use of higher order integration formulae will allow us to take a larger step size in Transient Simulation, as compared to the prevalent integration methods such as Trapezoidal Rule or Gear's method, used in SPICE like simulators.

We begin this chapter by looking at the Obreshkov Formula, and how it resolves the tension between Order and Stability in high-order integration methods. Section (3.2) will show how to adapt the Obreshkov Formula for solving MNA equations in Transient Simulation. Section (3.3) talks about efficient ways to factorize the Ja- 
cobian Matrix, while performing Newton iterations on Obreshkov based circuit MNA equations. Section (3.4) will talk about how the higher-order derivatives used in the Jacobian are calculated using rooted trees.

\subsection{Characteristics of the Obreshkov Formula}

The goal of the MNA equations in 2.25 is to evaluate the vector $\boldsymbol{x}(t)$ at discrete time points $t_{0}, t_{1}, t_{2}$ etc. The $l^{\text {th }}$ element of the vector $\boldsymbol{x}(t)$ will be represented as $x_{l}(t)$. This can be done by using an integration method known as the Obreshkov Formula presented in [2], which is as follows:

$$
\sum_{i=0}^{k}(-1)^{i} \alpha_{i, k, m} h^{i} x_{l, n+1}^{(i)}=\sum_{i=0}^{m} \alpha_{i, m, k} h^{i} x_{l, n}^{(i)}
$$

where

$$
\text { - } \quad h=t_{n+1}-t_{n}
$$

- $\quad x_{l, n+1}^{(i)}$ is an approximation of the $l^{t h}$ element of $\frac{d^{i}(x)}{d t^{i}}$ at $t=t_{n+1}$. That is:

$$
x_{l, n+1}^{(i)}=\left.\frac{d^{i}\left(x_{l}(t)\right)}{d t^{i}}\right|_{t=t_{n+1}}, l=1,2, . . N
$$

and $i=0$ represents the approximation obtained for the actual value of the waveform, that is, $x_{l, n+1}^{(0)}=x_{l}(t)$ at $t=t_{n+1}$. 
- The coefficient $\alpha_{i, k, m}$ is defined by the following expression:

$$
\alpha_{i, k, m}=\frac{(m+k-i) ! k !}{i !(m+k) !(k-i) !}
$$

The integers $k$ and $m$ used in (3.1) determine the stability and order of the Obreshkov method. The order of the method is determined by how closely $\boldsymbol{x}_{n+1}^{(0)}$ approximates the exact value $\boldsymbol{x}\left(t_{n+1}\right)$. It has been proved in $[2]$ that $\boldsymbol{x}_{n+1}^{(0)}$ approximates $\boldsymbol{x}\left(t_{n+1}\right)$ up to the order $k+m$, that is $\boldsymbol{x}_{n+1}^{(0)}-\boldsymbol{x}\left(t_{n+1}\right)=O\left(h^{k+m+1}\right)$, where $O\left(h^{p}\right)$ denotes terms that are proportional to $h^{q}, q \geq p$. It has also been proved in [2], that the stability of the method is not dependent on the order $k+m$, as long as $k-2 \leq m \leq k$. This helps us to take larger time steps without the risk of losing stability. As seen in section 2.3.4, the Gear's method that is used in today's circuit simulators loses A-stability for integration methods of order higher than 2.

\subsection{Obreshkov based Circuit Simulation}

It was discussed in section (2.5), that the time domain behaviour of circuits can be modeled using Modified Nodal Analysis, which gives the system of equations given in 2.25. If Obreshkov Formula is applied to this MNA system, we get the following 
system of equations 7 :

$$
\tilde{\boldsymbol{C}} \boldsymbol{\xi}_{n+1}+\tilde{\boldsymbol{G}} \boldsymbol{\xi}_{n+1}+\tilde{\boldsymbol{\rho}}_{n+1}=\tilde{\boldsymbol{u}}_{n+1}
$$

where the above matrices $\tilde{\boldsymbol{C}}$ and $\tilde{\boldsymbol{G}}$ are constructed from $\boldsymbol{C}$ and $\boldsymbol{G}$ as follows:

$$
\begin{aligned}
& \tilde{\boldsymbol{C}}=\frac{1}{h}\left[\begin{array}{ccccc}
\mathbf{0} & \boldsymbol{C}_{N \times N} & \ldots & \ldots & \mathbf{0} \\
\mathbf{0} & \mathbf{0} & \boldsymbol{C} & \ldots & \mathbf{0} \\
\vdots & \vdots & \ddots & \ddots & \vdots \\
\mathbf{0} & \mathbf{0} & \ldots & \mathbf{0} & \boldsymbol{C} \\
\mathbf{0} & \mathbf{0} & \ldots & \ldots & \mathbf{0}
\end{array}\right]_{(k+1) N \times(k+1) N} \\
& \tilde{\boldsymbol{G}}=\left[\begin{array}{ccccc}
\boldsymbol{G}_{N \times N} & \mathbf{0} & \ldots & \ldots & \mathbf{0} \\
\mathbf{0} & \boldsymbol{G} & \mathbf{0} & \ldots & \mathbf{0} \\
\vdots & \vdots & \ddots & \ddots & \vdots \\
\mathbf{0} & \mathbf{0} & \ldots & \boldsymbol{G} & \mathbf{0} \\
\alpha_{0, k, m} \boldsymbol{I} & -\alpha_{1, k, m} \boldsymbol{I} & \ldots & \ldots & (-1)^{k} \alpha_{k, k, m} \boldsymbol{I}
\end{array}\right]_{(k+1) N \times(k+1) N}
\end{aligned}
$$

where $\boldsymbol{I} \in \mathfrak{R}^{N \times N}$ is an Identity Matrix. Also,

$$
\boldsymbol{\xi}_{n+1}=\left[\begin{array}{llll}
\boldsymbol{x}_{n+1}^{(0)^{\top}} & h \boldsymbol{x}_{n+1}^{(1)^{\top}} & \ldots & h^{k} \boldsymbol{x}_{n+1}^{(k)^{\top}}
\end{array}\right]^{\top}
$$


where $\boldsymbol{x}_{n+1}^{(i)}=\left[\begin{array}{llll}x_{1, n+1}^{(i)} & x_{2, n+1}^{(i)} & \ldots & x_{N, n+1}^{(i)}\end{array}\right]^{\top}$. The vector $\tilde{\boldsymbol{\rho}}_{n+1}$ is defined as

$$
\tilde{\boldsymbol{\rho}}_{n+1}=\left[\begin{array}{lllll}
\boldsymbol{\rho}_{n+1}^{(0)^{\top}} & \boldsymbol{\rho}_{n+1}^{(1)^{\top}} & \ldots & \boldsymbol{\rho}_{n+1}^{(k-1)^{\top}} & 0^{\top}
\end{array}\right]^{\top}
$$

where $\boldsymbol{\rho}_{n+1}^{(i)}=\left.h^{i} \frac{d^{i} \boldsymbol{f}(\boldsymbol{x}(t))}{d t^{i}}\right|_{t=t_{n+1}}$, and T denotes the transpose operator.

The source vector $\tilde{\boldsymbol{u}}_{n+1}$ is defined in terms of the $h$-scaled derivatives of the independent sources in the circuit, and the previous state variables as follows:

$$
\tilde{\boldsymbol{u}}_{n+1}=\left[\begin{array}{lllll}
\boldsymbol{u}_{n+1}^{(0)^{\top}} & \boldsymbol{u}_{n+1}^{(1)^{\top}} & \ldots & \boldsymbol{u}_{n+1}^{(k-1)^{\top}} & \sum_{j=0}^{m} \alpha_{i, m, k} h^{j} \boldsymbol{x}_{n}^{(j)^{\top}}
\end{array}\right]^{\top}
$$

where $\boldsymbol{u}_{n+1}^{(i)}=\left.h^{i} \frac{d^{i} \boldsymbol{u}(t)}{d t^{i}}\right|_{t=t_{n+1}}$.

The nonlinear algebraic system of (3.4) can be solved using Newton iterations. The Jacobian matrix is shown below:

$$
\tilde{\boldsymbol{J}}=\left[\begin{array}{ccccc}
\boldsymbol{G}+\boldsymbol{J}_{0,0} & \frac{1}{h} \boldsymbol{C}+\boldsymbol{J}_{0,1} & \boldsymbol{J}_{0,2} & \ldots & \boldsymbol{J}_{0, k} \\
\boldsymbol{J}_{1,0} & \boldsymbol{G}+\boldsymbol{J}_{1,1} & \frac{1}{h} \boldsymbol{C}+\boldsymbol{J}_{0,1} & \ldots & \boldsymbol{J}_{1, k} \\
\vdots & \vdots & \ddots & \ddots & \vdots \\
\boldsymbol{J}_{k-1,0} & \boldsymbol{J}_{k-1,1} & \ldots & \ldots & \frac{1}{h} \boldsymbol{C}+\boldsymbol{J}_{k-1, k} \\
\alpha_{0, k, m} \boldsymbol{I} & -\alpha_{1, k, m} \boldsymbol{I} & \ldots & \ldots & (-1)^{k} \alpha_{k, k, m} \boldsymbol{I}
\end{array}\right]_{(k+1) N \times(k+1) N}
$$

where $\boldsymbol{J}_{i, j}=\frac{\partial \boldsymbol{f}_{n+1}^{(i)}}{\partial \boldsymbol{x}_{n+1}^{(j)}} h^{i-j}$.

It can be seen that if $i<j, J_{i, j}=0$. This transforms the Jacobian to a lower 
block Hessenberg matrix. If we compare the size of the Jacobian matrix in 3.10 obtained from the Obreshkov formula, to the size of the usual Jacobian matrix in (2.28), we observe that the Obreshkov based Jacobian matrix is $(k+1)$ times larger than the usual Jacobian matrix. This means that at each time step, more work needs to be performed with the Obreshkov based methods. However, with the Obreshkov method, we also save on the number of time steps to work on, which offsets the cost per time step, and gives significant savings in the overall transient simulation [7].

\subsection{Factorization of the Jacobian Matrix}

As mentioned in the previous section, the Obreshkov based Jacobian matrix is $k+$ 1 times larger than the ordinary MNA matrix. Hence the cost of factorizing the Obreshkov based matrix is higher than that in MNA. Several ideas were presented in [7] to mitigate this cost. These ideas are presented below.

\subsubsection{Structural properties of the Jacobian matrix}

The Jacobian matrix in $(3.10)$ is repetitive in nature. This can be inferred by ex-

panding the Jacobian matrix $\frac{\partial \boldsymbol{f}}{\partial \boldsymbol{x}}$, of the nonlinear part in a Taylor series expansion at $t=t_{n+1}$ as follows:

$$
\frac{\partial \boldsymbol{f}}{\partial \boldsymbol{x}}=\sum_{u=0}^{\infty} \frac{1}{u !} \boldsymbol{R}_{n+1}^{(u)} \tau^{u}
$$


where $\tau=\frac{t-t_{n+1}}{h}$ and $\boldsymbol{R}_{n+1}$ is the matrix valued Taylor series coefficients of the expansion. We can infer that $\boldsymbol{J}_{0,0}$ is the same as $\frac{\partial \boldsymbol{f}}{\partial \boldsymbol{x}}$. In [7], it is further shown that

$$
\boldsymbol{J}_{i, j}=h^{i-j} \frac{i !}{j !} \frac{1}{(i-j) !} \boldsymbol{R}_{n+1}^{i-j}
$$

Thus the 2D array of matrices in (3.10), i.e. $\boldsymbol{J}_{i, j}$ can be represented by a single dimension array of matrices $\boldsymbol{R}_{n+1}^{u}$, for $i=0,1, \ldots, k-1$, which are obtained from the Taylor series coefficients of $\frac{\partial \boldsymbol{f}}{\partial \boldsymbol{x}}$ matrix 19 . Equation 3.12 can be further simplified to

$$
\tilde{\boldsymbol{J}}=\left[\begin{array}{ccccc}
\boldsymbol{G}+\boldsymbol{R}_{n+1}^{(0)} & \frac{\boldsymbol{C}}{h} & & & \\
h \boldsymbol{R}_{n+1}^{(1)} & \boldsymbol{G}+\boldsymbol{R}_{n+1}^{(0)} & \frac{\boldsymbol{C}}{h} & & \\
\vdots & \vdots & \ddots & & \\
& & & & \\
& & \ldots & \boldsymbol{G}+\boldsymbol{R}_{n+1}^{(0)} & \frac{\boldsymbol{C}}{h} \\
\alpha_{0, k, m} \boldsymbol{I} & -\alpha_{1, k, m} \boldsymbol{I} & \ldots & \ldots & (-1)^{k} \alpha_{k, k, m} \boldsymbol{I}
\end{array}\right]_{(k+1) N \times(k+1) N}
$$

\subsubsection{Reducing the size of the Jacobian matrix}

The size of the matrix in equation 3.13 can be reduced from $(k+1) N \times(k+1) N$ to $k N \times k N$, without affecting the non-zero pattern of the matrix. $x_{k, n+1}$ can be written 
according to the lower order terms in the Obreshkov formula as follows:

$$
h^{k} \boldsymbol{x}_{k, n+1}=\frac{1}{(-1)^{k} \alpha_{k, k, m}} \sum_{i=0}^{m} \alpha_{i, m, k}\left(h^{i} \boldsymbol{x}_{n}^{(i)}\right)-\frac{1}{\alpha_{k, k, m}} \sum_{i=0}^{k-1} \alpha_{i, k, m}\left(h^{i} \boldsymbol{x}_{n+1}^{(i)}\right)
$$

Substituting (3.14) in (3.4) we get:

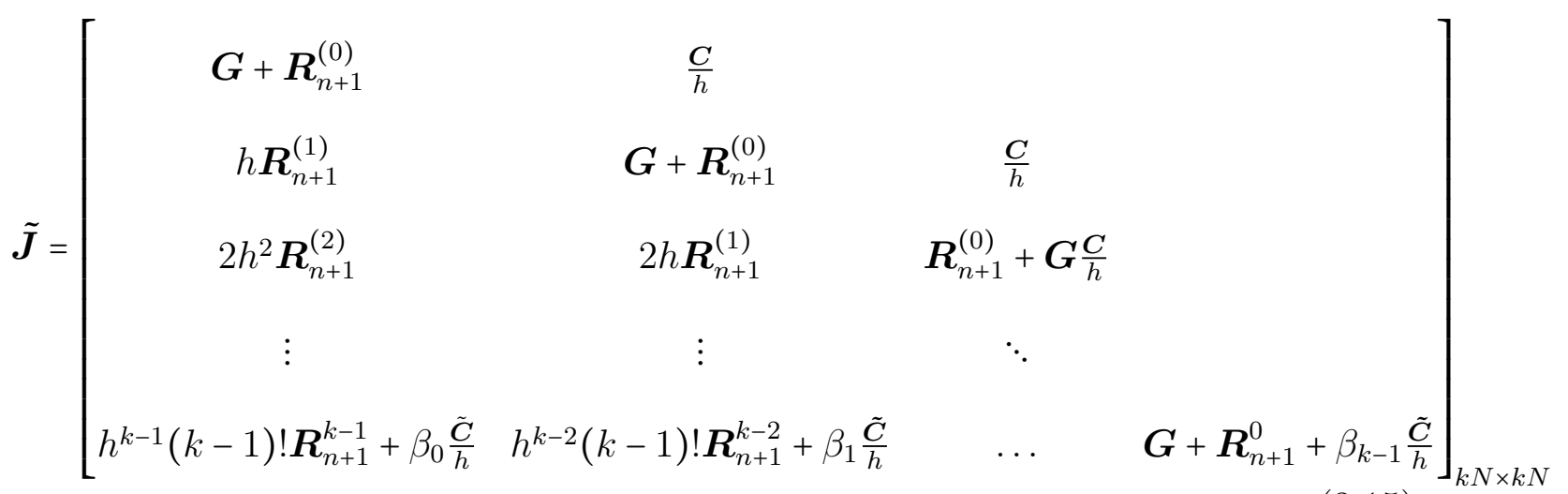

where $\beta_{i}=(-1)^{k+i+1} \frac{\alpha_{i, k, m}}{\alpha_{k, k, m}}$.

\subsubsection{Block Factorization of the Jacobian Matrix}

The technique of Block LU Factorization [7] can be used, to speed up the factorization of the Jacobian matrix in (3.15). This can be done in a two step process. The first step involves sparse ordering of the matrix, and the second step involves numerical computations using partial pivoting [20].

For the first step of sparse ordering, it was observed that the sparsity pattern of the $k N \times k N$ Jacobian matrix in (3.15), is identical to the sparsity pattern of the 
$N \times N$ sparse Jacobian matrix arising in traditional lower order integration methods like TR. So we construct an artificial sparse $N \times N$ matrix, and do sparse ordering on it. Also it is observed that, for all time points in the transient analysis, and for each Newton iteration for every such time point, the sparsity pattern of the matrix does not change. So the sparse ordering of the Jacobian matrix has to be done only once, at the beginning of the simulation. The cost of doing the sparse ordering, is found to be the same as that for the lower order method [7]. We can treat the Jacobian as a matrix of $k \times k$ blocks, where block is of size $N \times N$. The sparse ordering step can then be represented as:

$$
\hat{\boldsymbol{J}}=\boldsymbol{P} \tilde{\boldsymbol{J}} \boldsymbol{P}^{\top}
$$

Now before we perform the numerical analysis step for each time point, and each Newton iteration, we need to construct the Jacobian matrix, given the ordering of the rows and columns from the previous sparse ordering result. We can construct the Jacobian matrix by building it block by block. Each $N \times N$ block of the matrix can be of one of the following forms:

- A diagonal block, of the form $g \boldsymbol{I}$. These blocks can arise if nodes $a$ or $b$ or both are connected to linear memoryless (resistive) elements.

- A block of the form $\frac{c}{h} \boldsymbol{B}$, where $\boldsymbol{B}$ is the matrix of the companion form. This type of matrix can arise if either $a$ or $b$ or both nodes are 
connected to linear memory (capacitive/inductive) elements.

$$
\boldsymbol{B}=\left[\begin{array}{cccc}
0 & 1 & & \\
\vdots & \ddots & \ddots & \\
0 & \ldots & 0 & 1 \\
\beta_{1} & \beta_{2} & \ldots & \beta_{k-1}
\end{array}\right]
$$

- $\quad$ If either node $a$ or $b$ or both are connected to nonlinear memoryless elements, then a lower triangular matrix of the following form can occur:

$$
\left[\begin{array}{cccc}
r_{0} & & & \\
h r_{1} & r_{0} & & \\
\vdots & & \ddots & \\
h^{k-1}(k-1) ! r_{k-1} & & \ldots & r_{0}
\end{array}\right]
$$

- If either or both of the nodes are connected to more than one of the above types, then a lower Hessenberg matrix will occur as follows

$$
\left[\begin{array}{ccccc}
g+r_{0} & \frac{c}{h} & & \\
h r_{1} & g+r_{0} & \frac{c}{h} & & \\
\vdots & & \ddots & \ddots & \\
& & & & \\
& & \cdots & & g+r_{0}-\beta_{k-1} \frac{c}{h}
\end{array}\right]
$$


Thus looking at the row and column indices, and looking at the circuit elements connected to those nodes from the netlist, it is possible to construct the entire Jacobian matrix $\hat{\boldsymbol{J}}$ in equation 3.16 .

Once the matrix $\hat{\boldsymbol{J}}$ is constructed, it can be factorized using partial pivoting according to the algorithm mentioned in 20. Since $\hat{\boldsymbol{J}}$ is a block $k \times k$ matrix, the pivot 21] for partial pivoting can be chosen as the block with the largest determinant in the column. Here, instead of calculating the entire determinant of the block, which is known to have cubic complexity, we merely generate an estimate of the actual determinant by multiplying all the diagonal elements of the block. Since the pivoting step requires only comparing the determinants of each block, an approximation of the determinant is enough for the comparison. Thus numerical analysis at each time step and iteration can be performed, which gives the required LU factorization of the Jacobian matrix.

\subsection{Evaluating derivatives of nonlinear vector}

The nonlinear algebraic system of (3.4) can be solved using Newton-Raphson (NR) iterations. The input to the NR method is an initial guess of unknowns, which in the case of $(3.4)$ is given by $\boldsymbol{\xi}_{n+1}$. This is a $k(N+1)$ element vector, which contains the 0 to $k-1$ derivatives of $\boldsymbol{f}(\boldsymbol{x}(t))$. The output of an iteration of the Newton method, is a new value of $\boldsymbol{\xi}_{n+1}$, which it computes using the vector $\tilde{\boldsymbol{\rho}}_{n+1}$, and the Jacobian $\tilde{\boldsymbol{J}}$. 
Let us say the $q^{\text {th }}$ element in $\boldsymbol{f}(\boldsymbol{x}(t))$ represents the diode current

$$
f_{q}(\boldsymbol{x}(t))=I_{s}\left(e^{\frac{x_{q}(t)-x_{p}(t)}{V_{T}}}-1\right)
$$

In order to find higher order derivatives of this exponential diode current, we represent the equation (3.20) as a rooted tree shown in Figure 5.5. A rooted tree is a Directed Acyclic Graph, which has the following types of nodes:

- $\quad$ Leaf Nodes: These nodes do not have any outgoing edges, hence they are the terminating nodes of the tree. They represent the independent variables in the expression such as $x_{q}(t), x_{p}(t)$. They can also represent constants like the term $I_{s}$ in equation (3.20). Calculating the derivatives of these nodes is trivial, because if the node represents a constant, the derivative is 0 , and if the node represents an independent variable, then it's derivatives are already given in the initial guess $\boldsymbol{\xi}_{n+1}$.

- Regular Nodes: These are the intermediate nodes, and represent the functions or operations like the exponentiation function or the division operator. These functions usually take atleast one input to do their job, and they have an outgoing edge to each input that the function depends on. The derivatives of these nodes can be calcu- 
lated using the derivatives of the nodes they depend on (their children nodes). This is explained below.

- Root node: This represents the entire nonlinear expression such as the term $f_{q}(\boldsymbol{x}(t))$ in equation 3.20 .

As mentioned earlier, computing derivatives of the children nodes is trivial. To illustrate the computation of derivatives of the regular nodes, let us consider a regular node such as the exponentiation node in the above equation. Expanding $x(t)$ and $e^{x(t)}$ in their Taylor series expansion around $t=t_{n+1}$, we get:

$$
\begin{aligned}
& x(t)=\sum_{i=0} u_{i} \tau^{i} \\
& e^{x(t)}=\sum_{i=0} v_{i} \tau^{i}
\end{aligned}
$$

where

$$
\begin{gathered}
u_{i}=\frac{1}{i !} h^{i} \frac{d^{i}(x)}{d t^{i}} \\
v_{i}=\frac{1}{i !} h^{i} \frac{d^{i}\left(e^{x(t)}\right)}{d t^{i}} \\
\tau=\frac{t-t_{n+1}}{h}
\end{gathered}
$$


It can be shown that $[7]$ the $i^{\text {th }}$ derivative of the exponential function can be computed as:

$$
v_{i}=\frac{1}{i} \sum_{m=0}^{i-1}(i-m) v_{m} \times u_{i-m}
$$

where $v_{m}$ is the parent derivative and $u_{i-m}$ is the child derivative.

Thus the $h^{i}$ scaled derivatives of the parent can be be obtained from the $h^{i}$ scaled derivatives of the children nodes, and the $h^{p}$ scaled derivatives of the parent nodes, where $p<i$. Similar expressions can be derived for other types of operations.

Finally, the $h^{i}$ scaled derivatives of the root node, i.e. $h^{i} f_{q, n+1}^{(i)}$, can be computed using Post-Order Depth First Traversal [22]. In this approach, the derivatives of all the child nodes are computed recursively to begin with, then these derivatives of the children are used to calculate the derivatives of the regular node or the root node.

\subsection{Discussion}

This chapter presented an overview of the Obreshkov based time domain simulation. Since the Obreshkov formula uses high-order derivatives, a critical aspect in this high-order simulation method is the construction of the nonlinear $\tilde{\boldsymbol{\rho}}$ vector and the Jacobian matrix $\tilde{\boldsymbol{J}}$, which are constructed by calculating the high-order derivatives of $\boldsymbol{f}(\boldsymbol{x}(t))$. If we express the nonlinear terms in $\boldsymbol{f}(\boldsymbol{x}(t))$ as rooted trees, then it can facilitate the computation of high-order derivatives. 
In the ensuing chapters of this thesis, a framework is developed to incorporate the generic SPICE device models in the high-order Obreshkov based method. SPICE models are the industry standard way of modeling generic nonlinear devices like diodes and transistors, for their use in time domain simulation. Incorporating these SPICE device models in Obreshkov based transient simulation, will make this higher order integration method more useful and reliable in the industry.

The process of incorporating SPICE device models will involve understanding the structure of the SPICE device model source code, parsing this source code for relevant details, and converting the nonlinear element model to a rooted tree, so that higher order derivatives can be calculated for the Obreshkov based method.

Chapter 4 in this thesis provides an overview on how the SPICE device model source code is structured, and identifies the parts of that source code which model the memoryless, memory, and nonlinear elements of the device models which are relevant for Obreshkov based simulation. Chapter 5 explains the technique of parsing the $\mathrm{C}$ source code of the SPICE device model, and how to build a rooted tree for the nonlinear elements. Once the rooted tree has been generated, it can be used to calculate the Jacobian $\tilde{\boldsymbol{J}}$ of the Obreshkov based method. 


\section{Chapter 4}

\section{Structure of the SPICE device model source}

\section{code}

The previous chapter described the process of computing the high-order derivatives of nonlinear function $f(x)$, by traversing its rooted tree. These high-order derivatives are used to populate the Jacobian matrix $\tilde{\boldsymbol{J}}$ shown in equation 3.15 . So, in order to use SPICE based models of nonlinear devices, in Obreshkov based methods, we need to extract the conductance and capacitive effects of the device from the SPICE model. More importantly, we need to develop a way of generating a rooted tree representation of the nonlinear current $f(x)$ from the SPICE based models.

In general, there is a common pattern as to how any device model is loaded in SPICE like simulator. The goal of this chapter is to describe this pattern. The next chapter will then present the framework that builds on that common pattern to 
generate the rooted trees in order to compute the high-order derivatives.

\subsection{Loading a Device model in SPICE}

This section starts out by first clarifying what is meant by the notion of loading a device model in SPICE. This notion refers to the process of using the device model to populate the entries of the Jacobian matrix $\boldsymbol{J}$ and the RHS vector $\boldsymbol{\phi}$ in equation (2.27). A good understanding of this process of loading a device model in SPICE is required, so that we can intercept this process to extract the data required for Obreshkov based method.

The process of loading a device model in SPICE involves several stages, and data extracted from the netlist flows through different data structures before being loaded in to the $\boldsymbol{J}$ and $\boldsymbol{\phi}$ matrices. Figure (4.1) shows the most important data structures involved, and enumerates the steps in which data flows through these data structures.

All devices in SPICE are instances of a master data structure, called SPICEdev. This structure is the only interface to the device, and is the only externally visible portion of the device implementation code. It contains pointers to all the functions supported by the device. All the interaction of SPICE with device models is through the function pointers declared in this structure.

The Core SPICE engine maintains an array of SPICEdev* pointers called DEVices. There is one and only one SPICEdev* pointer for each type of device. As can be seen 


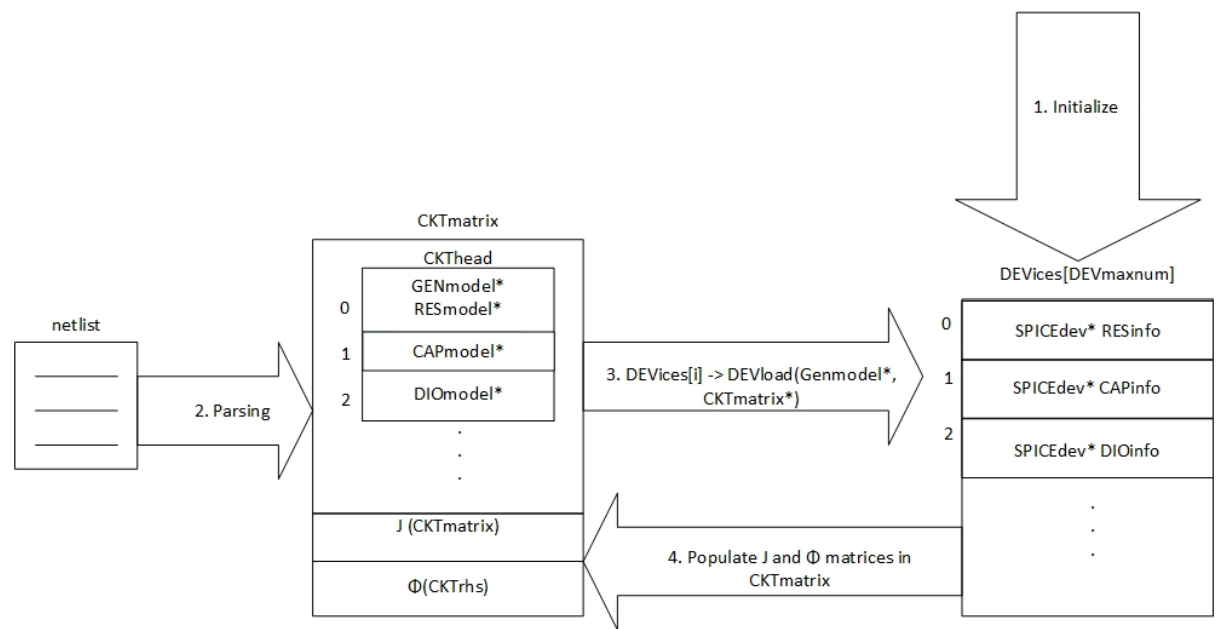

Figure 4.1: Data structures involved in loading SPICE device models

in Figure 4.1), there is only one SPICEdev* pointer for resistor (called RESinfo), only one SPICEdev* pointer for a diode (called DIOinfo) etc. Moreover, each type of device has a unique index in the DEVices array. In order to work with any type of device, the SPICE core engine has to know the position of the SPICEdev* pointer for that device type in the DEVices array. For example, to ask for info about diode, if the index of SPICEdev* pointer for diode in the DEVices array is 12 , then we can execute DEVices[12]->DEVask(). We will look at the SPICEdev structure in detail later in Section 4.2.3. When SPICE starts up, a unique instance of SPICEdev* is created for each type of device, and loaded into the DEVices array. This is shown by step 1 in Figure (4.1). The maximum number of different types of electronic devices that the simulator knows of, is called DEVmaxnum.

The process of loading a device model in SPICE begins when the user provides 
a netlist to represent the circuit to be simulated. SPICE parses this netlist to find out the devices in the circuit, and their interconnection. The device information extracted from the netlist during the parsing phase is stored in two device specific data structures called GENmodel and GENinstance. This is shown by step 2 in Figure 4.1). These will be explained in Section 4.2).

The CKTcircuit structure stores all the known information about the circuit being simulated. When the parsing stage ends, the GENmodel* and GEninstance* data extracted from the netlist, is stored in the CKTcircuit structure in an array called CKThead. CKThead is an array of DEVmaxnum instances of GENmodel*, one for each type of device. The CKT circuit structure also stores Jacobian matrix $\boldsymbol{J}$ as a sparse matrix called CKTmatrix, and the RHS vector $\boldsymbol{\phi}$ called CKTrhs. The device loading phase takes the data in the CKThead array and populates the CKTmatrix and CKTrhs members of the CKTcircuit. This is shown in step 3 and 4 of Figure (4.1). The CKT circuit structure will be discussed in Section 4.2.4).

During transient analysis, steps 3 and 4 in Figure (4.1) are repeated at each time point. Once the $\boldsymbol{J}$ matrix and $\boldsymbol{\phi}$ vector are populated for a time point, SPICE can then perform LU decomposition on the $\boldsymbol{J}$ matrix to solve the Newton-Raphson iteration equation (2.27). 


\subsection{Data Structures to hold netlist data}

Listing (4.1) shows an example of a SPICE netlist for a full wave bridge rectifier circuit.

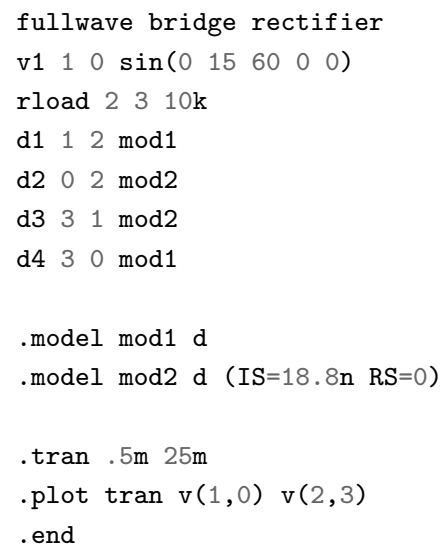

Listing 4.1: SPICE netlist for Bridge Rectifier

In SPICE, a device is specified in the circuit netlist as a model. A device used in a circuit can have several different models for simulation, and each model can have 0 to many instances of that model. As can be seen in Listing (4.1), for a diode, there are two diode models in the netlist, namely mod1 and mod2. Diode model mod1 has two instances in the circuit, namely $\mathrm{d} 1$ and $\mathrm{d} 4$, and diode model mod2 has two instances in the circuit, namely $\mathrm{d} 2$ and $\mathrm{d} 3$. This fact is captured by the GENmodel and GENinstance data structures [23]. There is a inheritance hierarchy for models and instances, and is shown in Figure 4.2. GEN refers to general, and represents the base-struct for a model or instance of a general device. A specific device model, 
such as a resistor model, has to inherit from the general model.
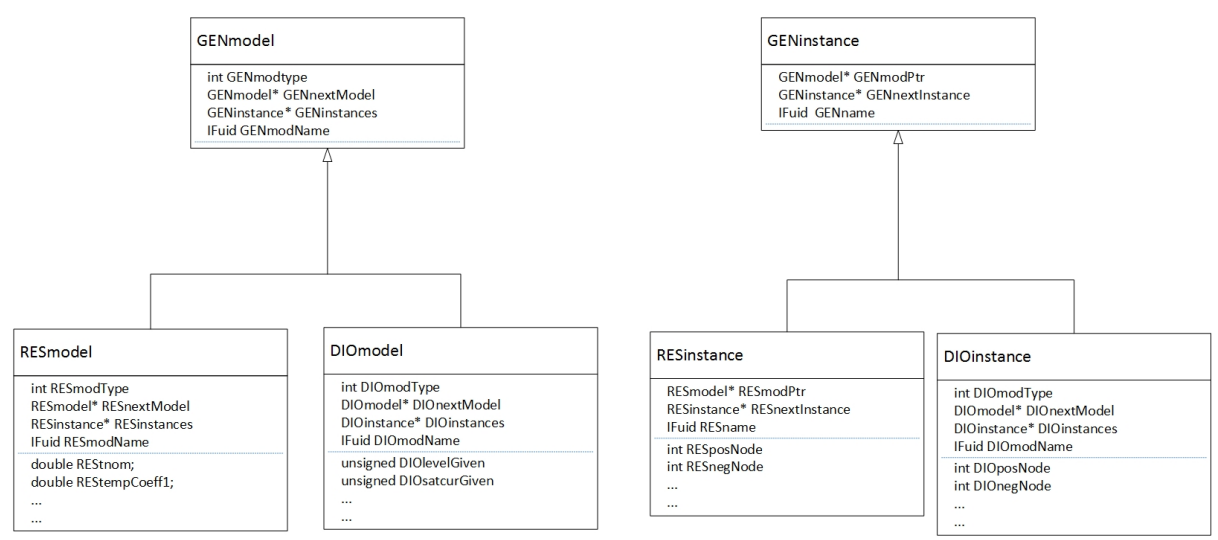

Figure 4.2: Inheritance hierarchy of GENmodel and GENinstance

The device model source code is written in $\mathrm{C}$, and there is no language support for inheritance in $\mathrm{C}$, as is available in $\mathrm{C}++$. So the class hierarchy of Figure 4.2 is implemented in $\mathrm{C}$ by mandating that the base-struct and derived-struct share common member variables in the beginning. The derived-struct is then free to add it's own member variables that it needs, to store the device information from the netlist. This will be explained in more detail in Section 4.2.1) and Section 4.2.2).

This inheritance hierarchy implemented with structs in C, allows a base-struct to appear in place of derived-struct, using pointer type-casting as shown in Listing (4.2):

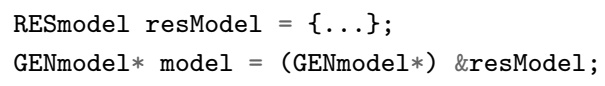

Listing 4.2: Casting a derived-struct pointer to base-struct pointer 


\subsubsection{GENmodel}

The model data structure stores the parameters which are common to all the instances of that model. Since a model can have 0 to many device instances associated with it, each model data structure contains a linked-list of all the instances of that model. These are like the children of the model.

A type of device like a diode can have several models in the circuit, so the models are themselves in a linked list. Each model stores a pointer to the next model in the list, which is it's sibling. For the netlist in Listing (4.1), the linked list of models and instances will be structured as shown in Figure 4.3.

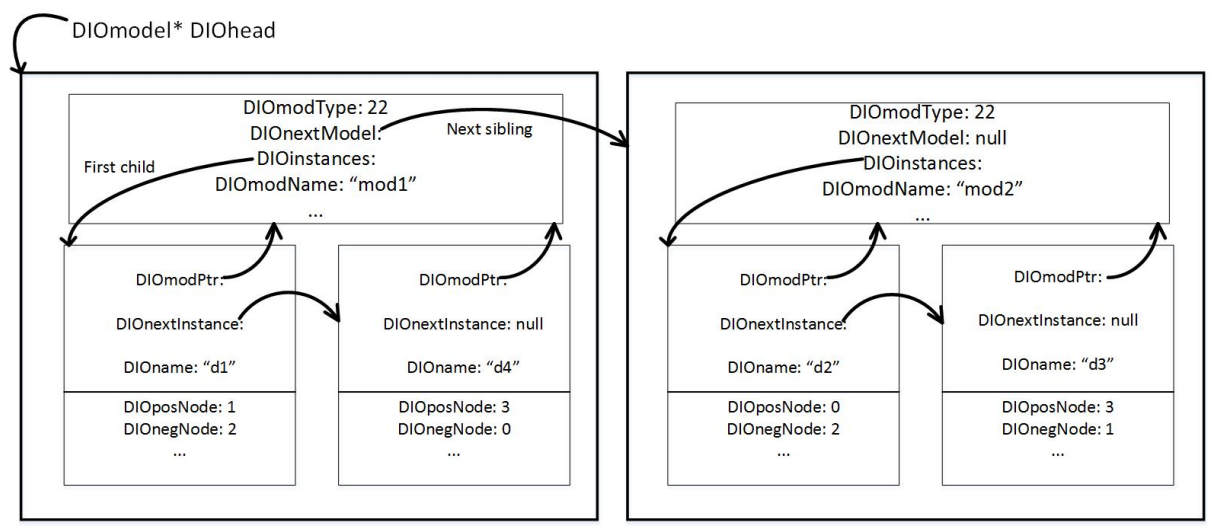

Figure 4.3: Linked List structure of Models and Instances for diodes in Listing (4.1)

Thus, given the head pointer to a netlist device model (DIOmodel* DIOhead in Figure (4.3), it is possible to discover all user-defined models for that type of device in the netlist, and the device instances associated with each such model.

To implement the inheritance hierarchy of models as shown in Figure (4.2), the 
base-struct, GENmodel, must have standard header of four elements in exactly the same order [23]. This header contains the mandatory part of the model structure. The standard header for a model is shown in Listing 4.3 .

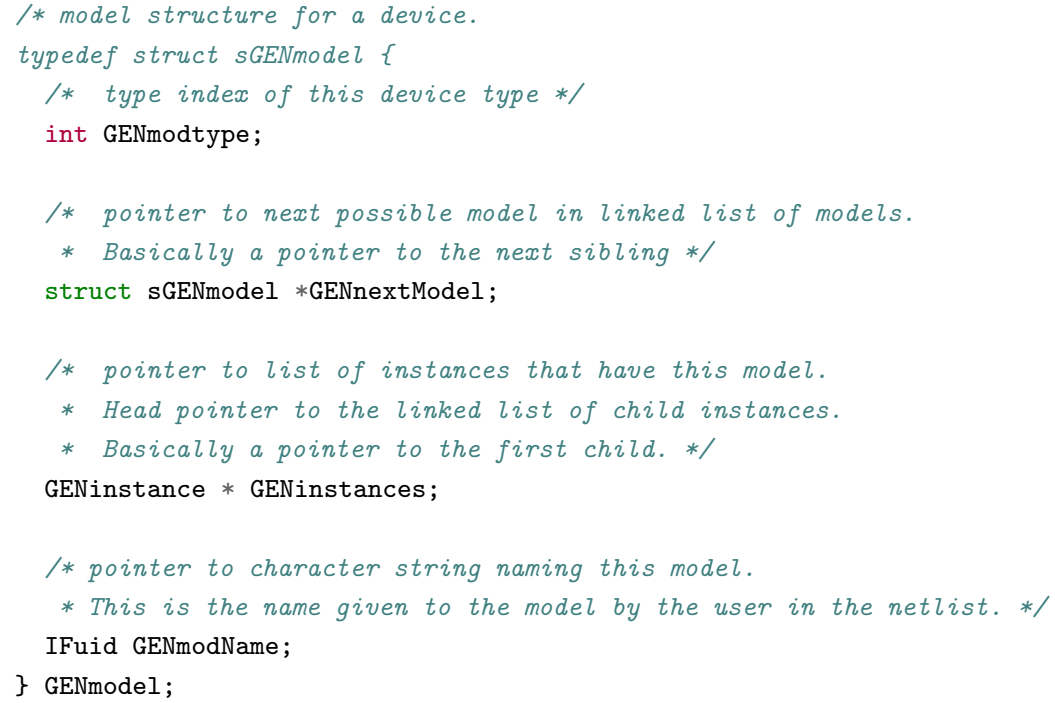

Listing 4.3: Definition of device model

An example of a struct derived from GENmodel as a base class, would be the DIOmodel. It is shown in Listing (4.4). It can be seen that the first four member variables of DIOmodel are same as the base struct GENmodel. The rest of the model structure is free to be defined by the implementor of the device, and contains the many model parameters that the device may need. 


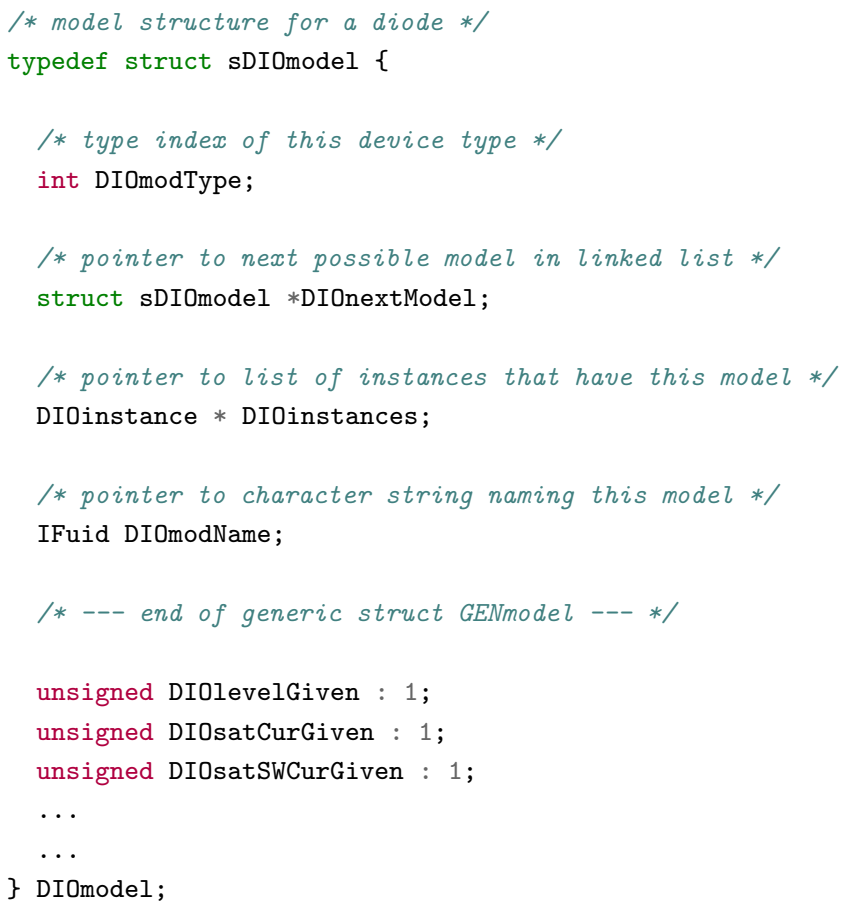

Listing 4.4: Definition of Diode model

\subsubsection{GENinstance}

The base-struct for an instance has a standard header of three mandatory elements. This is shown in Listing (4.5). The first three elements are mandatory. GenmodPtr is a back pointer to the model. GENnextInstance is a pointer to the next instance in the linked-list, and GENname is a name of this instance. Structs that derive from GENinstance as base class, add their own member variables after the mandatory section. These member variables start with the list of external nodes of this instance, in the same order that they appear in the netlist [23]. This is usually followed by the 
instance-specific parameters.

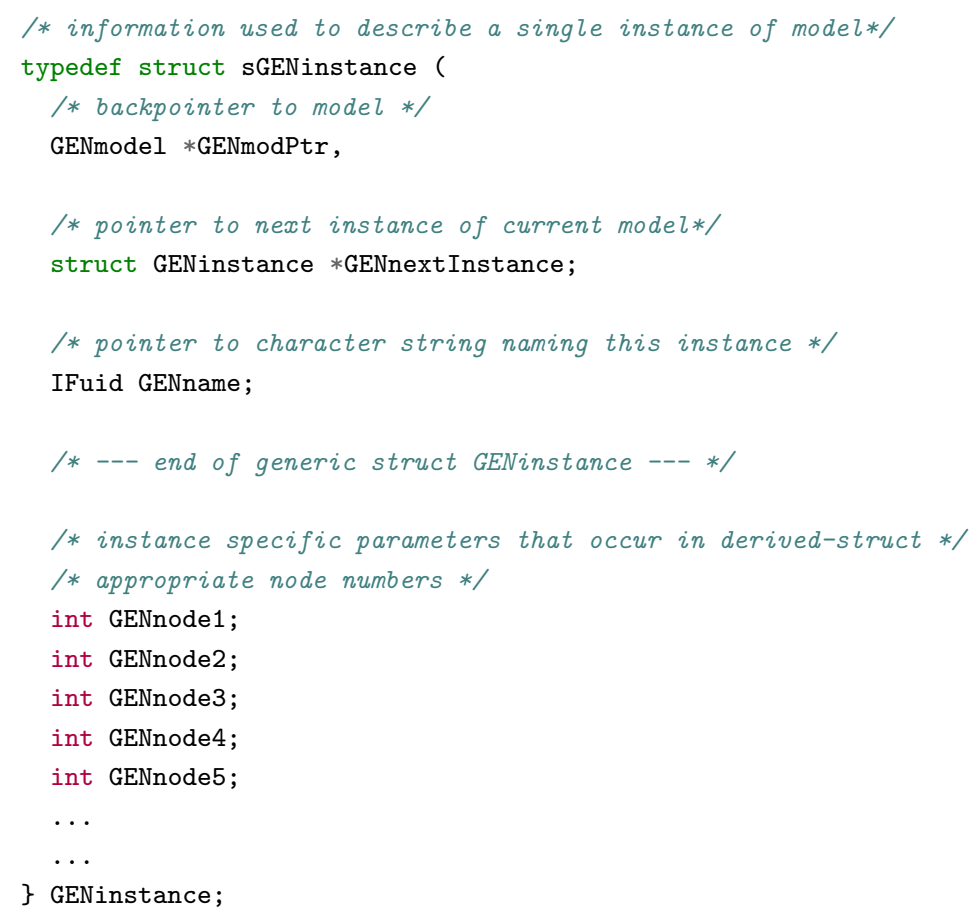

Listing 4.5: Definition of device instance

\subsubsection{The master data structure}

The overall data structure contains pointers to the functions that provide the functionality of the device. It also contains some parameters that are required by the functions to do their job. This master data structure contains all the data that is required to interface with the device model, and is the only externally visible interface to the device. It is shown in Listing (4.6).

The SPICEdev structure is divided in to two main parts. The first part, DEV public, 


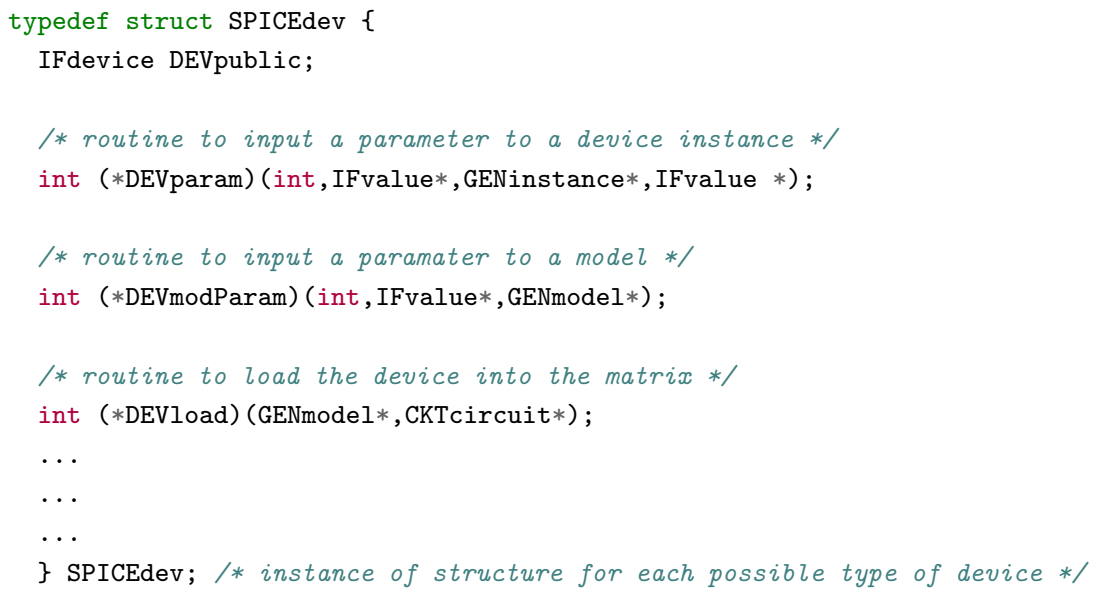

Listing 4.6: SPICEdev master data structure

is designed to comply with the requirements of the netlist parser. It provides information to the parser about the device parameters and their data types, the number of nodes and their names, and the name of the device. The IFdevice structure which defines the DEV public part can be found in Listing (4.7).

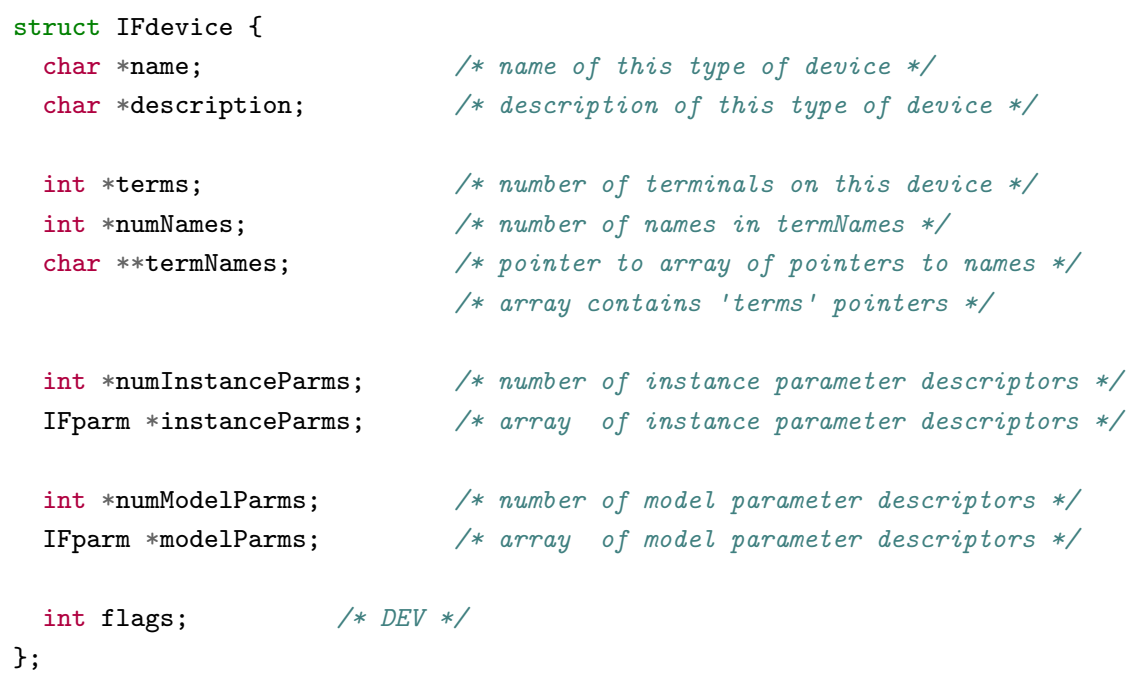

Listing 4.7: IFdevice structure that defines the device in SPICEdev 
The second part of the SPICEdev structure contains the pointers to the functions that provide the functionality of the device. Of these functions, the most important function is the DEVload, which loads the device model in the MNA matrices. This function will be discussed in detail in Section 4.3 , but as can be seen from listing 4.6 , the DEVload function takes a pointer to the device model GENmodel, and takes a pointer to the CKT circuit structure, which stores all the MNA matrices required for the circuit simulation. The job of the DEVload function is to perform steps 3 and 4 of Figure 4.1), i.e. to populate the MNA matrices $\boldsymbol{J}$ and $\boldsymbol{\phi}$ in CKTcircuit*, using the device models defined in CKThead. We will look at the structure of the DEVload function, after looking at the CKTcircuit structure. It is really this DEVload function that will enable us to generate the rooted tree, since it loads the device models in the MNA matrices.

\subsubsection{CKT circuit structure}

This structure describes the circuit that is being simulated, and contains the MNA matrices representing the circuit. The source code for this structure can be found in Appendix A. The CKTmatrix member variable contains the sparse Jacobian matrix ( $\boldsymbol{J}$ in Equation (2.27)). This matrix is populated directly by the device models in their load functions, depending on the type of integration method being used, either Trapezoidal rule or Gear's method. 
The $C K T r h s$ element stores the right hand side MNA vector ( $\phi$ in Equation (2.27)), that is being currently populated. The CKTrhsOld element holds the solution vector from the previous iteration or timepoint. There are eight one dimensional arrays from $C K$ Tstate 0 to $C K T$ state7, which store solution vectors for previous timepoints, required by Gear's method. The CKTstate 0 vector contains the data for the current time point, CKTstate 1 contains data for the first previous timepoint, and CKTstate 7 contains data for the 6 th previous time point. The simulator automatically shifts the data between time points, as the simulation progresses. The device model only has to keep an index into the states vector, to refer to data of a particular time point.

The simulator has a state machine, which controls the type of analysis being performed, such as AC, DC or Transient Analysis, and the states in the analysis, like handling the special case of the first iteration in DC, and Transient Analysis, Operating Point Analysis etc [23]. During the loading of the device model, the CKTmode also controls how the terminal voltage of the device instance is determined.

\subsection{DEVload function}

The task of populating the $\boldsymbol{J}$ and $\boldsymbol{\phi}$ matrices in Equation 2.27) using the GENmodel pointers extracted from the netlist, is performed by the DEVload function, registered in the SPICEdev structure for that device.

As can be seen in Equation (2.28), each device can contribute to the Jacobian 
through it's conductive effects, capacitive effects, or if it is a nonlinear element, then through the nonlinear current and it's derivative. These effects are modeled in the DEVload function using the conductive stamp, capacitive stamp, and nonlinear current stamp of the device as discussed in Section (2.6), and the Jacobian and RHS matrices in the CKT circuit structure are loaded.

In order to integrate SPICE device models in Obreshkov based method, we need to identify these stamps in the DEVload source code for SPICE device models. Once the stamps have been identified, the part of the stamp which is specific for the SPICE-integration methods and Newton-Raphson iteration need to be ignored, and the nonlinear current of the device, conductive and capacitive effects of the device need to be extracted. The following subsections will describe how to identify these stamps in the DEVload source code, and what part of the stamps is relevant for Obreshkov based method.

\subsubsection{Nonlinear behaviour}

In the DEVload function, a nonlinear device is modeled by the nonlinear device stamp discussed in Section (2.6.1). As can be seen from Equation 2.33), the nonlinear current $c_{d}$ only appears in the RHS vector. The derivative of the nonlinear current $g_{d}$ appears in both the LHS and RHS matrices, and it's multiplied by $v_{d}$ in the RHS. Thus one way to identify the nonlinear current contribution of the device model is 
to observe the part of the DEVload source code where the $\boldsymbol{J}$ and $\boldsymbol{\phi}$ matrices are being populated, locate the $g_{d}$ like elements that appear in population of both the CKTmatrix and CKTrhs matrices of CKT circuit. The nonlinear current $c_{d}$ is added to the $g_{d}$ like elements while populating the RHS matrix.

Once we identify the variable in the SPICE device model source code that represents the nonlinear current $c_{d}$, we can look up the definition of this variable in the source code, and build a rooted tree out of the definition, for integrating the nonlinear current in the Obreshkov-based simulation. We are only interested in extracting the definition of variable $c_{d}$. Variable $g_{d}$ is an artifact of Newton-Raphson iteration, and is of no interest to us in Obreshkov based simulation, because in the Obreshkov based method, we compute our own derivatives using the rooted tree of $c_{d}$.

\subsubsection{Capacitive behaviour}

The PN junctions in nonlinear elements like diodes and MOSFETs, show capacitive behaviour around the depletion region. This is especially true for the large signal model of the nonlinear elements, which is what is required for Transient Analysis of the nonlinear elements. The capacitive behaviour of devices is modeled by SPICE using capacitors, whose formulae are specified in the DEVload function. Once these capacitor values have been defined, they have to be loaded in the $J$ and $\phi$ matrices. Capacitors are loaded in SPICE, also using their companion model [16], which was 
discussed in Section (2.6.2). The values of the $g e q$ and $c e q$ variables in Figure (2.6) are determined by the NIintegrate function [23], which takes in the capacitor value and the charge on it, and outputs the $g_{e q}$ and $c_{e q}$ variables, based on the integration method being used. These values are then loaded in the Jacobian matrix. Since these variables are integration method dependent, these variables are not important for Obreshkov based method. We just need to determine the value of the capacitance from the DEVload function. Nevertheless, it is important to know what function these $g_{e q}$ and $c_{e q}$ variables perform, so that we can safely ignore their values while extracting Obreshkov related data.

For the sake of this thesis, we have ignored the Capacitances in the device model. These capacitance values can be parsed using the technique outlined in the next chapter, and integrated in the Obreshkov method. However, these capacitances being nonlinear, can be taken up as future work along the lines of 24 .

\subsubsection{Conductive behaviour}

The conductive contribution of a device is modeled in the DEVload function using the resistive stamp which was discussed in Section (2.6.3). The resistive stamp only contributes to the Jacobian matrix, and not to the RHS vector. Hence to extract the resistive elements from the DEVload source code, we can say that the variables which are used only to populate the Jacobian matrix, are the resistive elements in 
the model.

In the next section, we will look at the DEVload function for the SPICE diode model. This will illustrate how to extract Obreshkov related data from a SPICE device model for a nonlinear element.

\subsection{The SPICE diode model}

At the bottom of the DEVload function, the Jacobian matrix is populated by using the device node numbers as row and column index in the Jacobian matrix. For example, at the bottom of the DIOload function, which is the DEVload function for a diode, the code fragment given in Listing 4.8 can be found. The full source code for DIOload function is given in Appendix B.

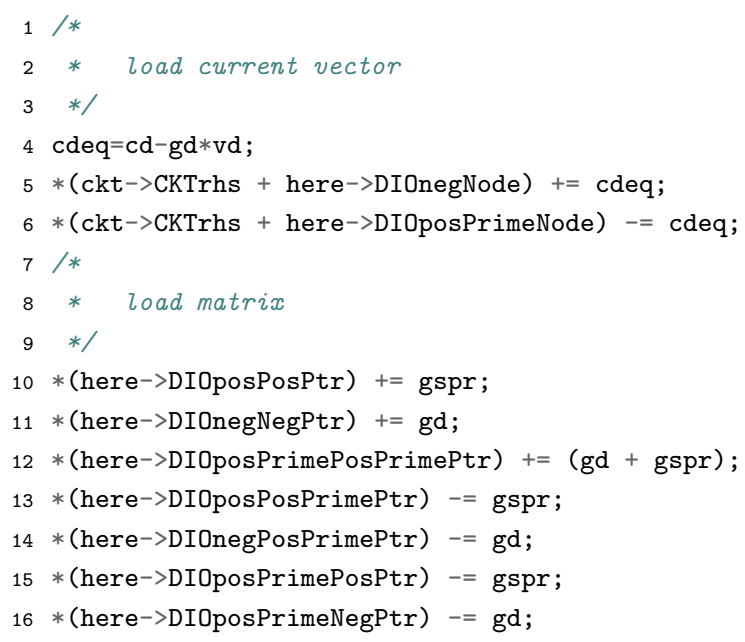

Listing 4.8: Loading of the SPICE diode model in DIOload function 
As seen in Listing 4.8, the (here->DIOposPosPtr) etc. are pointers to elements in the $c k t->C$ KTmatrix structure, that represents the Jacobian of the matrix. The $($ ckt->CKTrhs + here->DIOnegNode $)$ is a pointer into the RHS MNA vector. This amounts to populating the Jacobian matrix in the following way:

$$
\left[\begin{array}{ccc}
g s p r & -g s p r & \\
-g s p r & g d+g s p r & -g d \\
& -g d & g d
\end{array}\right]\left[\begin{array}{c}
v_{\text {pos }} \\
v_{\text {posPrime }} \\
v_{\text {neg }}
\end{array}\right]=\left[\begin{array}{c}
0 \\
-c d e q \\
+c d e q
\end{array}\right]
$$

From this pattern, we can infer that there is a resistance (conductance gspr) between nodes (pos) and (posPrime). This follows from the discussion in Section (2.6.3) and by observation that gspr occurs only while populating the Jacobian matrix. It makes no contribution to the RHS matrices.

We can also observe that the conductance $g d$ appears in both the Jacobian and RHS matrices. In the RHS vector, it appears in $c d e q$, since $c d e q=c d-g d v d$. Based on this observation, and the discussion of the companion form of the Newton-Raphson method in Section (2.6.1), we can say that $g d$ represents the derivative of the nonlinear current of the diode. The actual nonlinear current is given by the variable $c d$.

It can be seen in the source code in Appendix B, and line 366, that $g d$ is actually $g d=g d+g e q$. Here the $g d$ on the LHS represents the derivative of the nonlinear current. The variable geq represents the equivalent conductance of the capacitance 
companion model which is discussed in Section 2.6.2. The value of geq is loaded in the NIintegrate function, a call to which can be found in Appendix B, line 364. Here we can see that the values of $g e q$ and $c e q$ are loaded based on the variables capd and here->DIOcapCharge. Based on the definition of function NIintegrate, we can say that capd represents the capacitance of the diode, and here->DIOcapCharge represents the charge on the diode capacitance.

This tells us that the nonlinear current $c d$ is connected between nodes posPrime and neg. The capacitance capd is also connected between nodes posPrime and neg.

The rooted tree for the nonlinear current that we are interested in, can be generated by parsing the definition of the variable $c d$, written in the $\mathrm{C}$ language, in the DIOload function. The resistance and capacitance contribution can be obtained by parsing the definition of the gspr and capd variables.

It must be noted that SPICE does not require the $c d$ variable to represent the nonlinear current. It just happens to do this for the diode model. For the MOSFET model, there may be another variable, which will define the nonlinear currents. This variable which represents the nonlinear current will have to be determined from the pattern to populate the RHS at the bottom of the MOSload function. But once the variable is known, it should be possible to integrate the device in the Obreshkov based method.

From the source code of the DIOload function, the following SPICE diode model 
can be inferred:

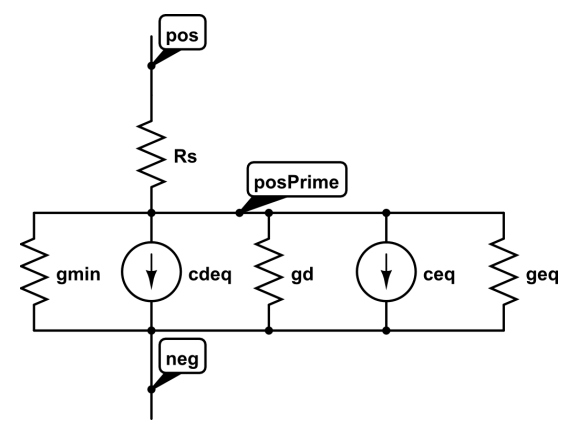

Figure 4.4: SPICE Diode Model

The nonlinear diode current ( $c d$ in the source code) models the exponential $i-v$ relationship. The rooted tree for $c d$ is quite elaborate, as it depends on the various ways in which the terminal potential difference of the diode is determined. In simplified terms, it is found to be:

$$
c d=I_{s}\left[e^{\frac{v_{p}-v_{n}}{n \times V_{T}}}-1\right]+v d \times c k t->C K T g \min
$$

Here $I_{s}$ is the saturation current, $c d$ is the nonlinear current of the diode, $v_{p}$ and $v_{n}$ are the voltages of the posPrime and negative nodes of the diode as represented in the DIOload source code. $V_{T}$ is the threshold voltage. ckt->CKTgmin is a small conductance that is added across the nonlinear current. This is mainly a convergence aid. In the reverse bias region, when $v d<-5 n V_{T}$, SPICE makes an assumption that the leakage current through the $\mathrm{PN}$ junction is equal to $I_{s}$. This is done to avoid calculating the exponential term [23]. In this region the equation for $c d$ then 
becomes:

$$
c d=-I_{s}+v d \times c k t->C K T g \text { min }
$$

Thus $I_{s}$ is the maximum current you can get from the diode under high reverse bias, as long as the diode does not exhibit a breakdown. In this region, if there was no $c k t->C K T$ gmin, then the conductance in the region would be 0 . To avoid this, the $c k t->C K T g m i n$ has been added.

The series resistance (gspr in the source code) models the total resistance of the metal leads which connect the diode to the rest of the circuit.

In order to simplify the job of generating and measuring the feasibility of using rooted trees, the capacitance has been ignored for the sake of this thesis. Modeling this dynamic behaviour of nonlinear devices can be taken up as future work, along the lines of the work published in [24].

In the next chapter, we will look at how to generate a rooted tree, for a variable like $c d$ defined in the C-language source code. Based on this rooted tree for the nonlinear current, and the series resistance, a new element can be declared in the Obreshkov method, called SPICE_DIODE, which will provide all the required SPICE diode contributions, using rooted trees. 


\section{Chapter 5}

\section{Generating the rooted tree from SPICE de- vice model source code}

In chapter (3), we saw that the higher-order derivative based Obreshkov method allows us to take bigger time steps in the transient simulation than traditional second order integration methods like Trapezoidal rule, without compromising on the accuracy of the simulation.

The formulation of the Jacobian matrix for Obreshkov based method requires the computation of derivatives of the nonlinear function $f(x)$ with respect to time $t$, for orders higher than 2. We said that it is possible to compute these higher order derivatives of $f(x)$, if the mathematical function represented by $f(x)$ is represented as a rooted tree. The rooted tree can be traversed from root to leaves, to calculate any order of derivatives of $f(x)$. 
In order for the Obreshkov based simulation method to be of pragmatic use, the method has to support SPICE based device models, especially for nonlinear devices. The quality of the simulation results depends directly on the quality of the device models used. The more faithfully the model represents the various characteristics of the device, the more accurately the simulation results will describe the real behaviour or the fabricated circuit, under the given input conditions. Generic SPICE device models have now become the industry standard in modeling the behaviour of devices, and they embody several years of dedicated research in modeling every characteristic and imperfection of the device. This chapter will therefore show, how a Generic SPICE device model can be incorporated in Obreshkov based circuit simulation.

\subsection{The problem statement}

The structure of the C-language source code for SPICE device models, especially for the diode, was outlined in Chapter 4. The objective of this chapter is to show how the C-language definition of the nonlinear current can be represented as a rooted tree, which can be incorporated in Obreshkov based circuit simulation. We want to find out whether the SPICE device model represented as a rooted tree, can give the same accuracy as the C-language based SPICE model, while also providing time savings over the traditional circuit simulation method. 


\subsection{Data Structure for the rooted tree}

A rooted tree, like the one shown in Figure 5.5, can be represented using the ObjectOriented paradigm in $\mathrm{C}++$. An abstract base class $i$ Expr can be defined, which will define general functionality that is common to all nodes of the rooted tree. For example, every node in the rooted tree should be able to calculate and return it's own derivative with respect to time. We can declare this functionality in the abstract base class $i$ Expr. Every sub-class of $i$ Expr, will then be forced to define a way of calculating and returning its own derivative.

Each node in the rooted tree, will have a concrete class that derives from the abstract base class $i$ Expr. A concrete class can be made for each possible type of node in the rooted tree, and the functionality unique to that type of node, can be added to the concrete class. For example, the multiplication operator takes two arguments. We can define a concrete class called mulTerm, which will have two children nodes to represent the two operands it requires. So the mulTerm class will have two iExpr pointers, each representing an operand of the multiplication term. On the other hand, an exponential term, takes only one operand, and should have only pointer to aniExprterm. However, both the mulTerm and expTerm classes need to have the functionality to calculate their moment. That functionality can hence go in the abstract base class. The leaf terms will also inherit from $i$ Expr, but 
they will have no child pointers. All nodes in the rooted tree are thus derived from iExpr. They can contain as manyiExprpointers, as many arguments they need.

The process of finding the higher order derivatives of $f(x)$ with respect to time, is called "moment". Hence we will call our function in the base class to find higher order derivatives as moment. It will take in the order of the derivative, and return the value of the derivative as a number of the double data type. This moment function in the abstract base class will be pure virtual, which means that every class that derives from $i$ Expr, will have to provide a definition for the moment function, using the child pointers that it has.

The moment function can be defined in a recursive fashion. We can call the moment function on the root node, and the root node will recursively call the moment function on its children, till the leaf nodes are reached. Computing derivatives of any order on the leaf node is trivial, since the leaf nodes are usually constants, or a function of time. These trivial derivatives of the leaf nodes, can be stored directly in the leaf nodes itself, while initializing them. If a leaf node is asked for derivative of order 1 , it will return it's derivative of order 1 to the parent node which called the moment function on that leaf node. The parent node will be an operator, like mulTerm. The parent term can then use the derivatives returned by the child terms, to compute its own derivative of order 1. A recursive formula can be developed for any parent node, to compute its derivatives, based on the derivatives of its children, as shown 
below (5.1). Once the entire first order derivative of the rooted tree is calculated, then the second order derivative can be calculated in the same fashion. This process will continue, till all derivatives of the rooted tree are computed. This formula can be coded in to the moment function of the parent node. Based on the above formulation, the recursive code for mulTerm can be expressed as shown in Listing (5.1).

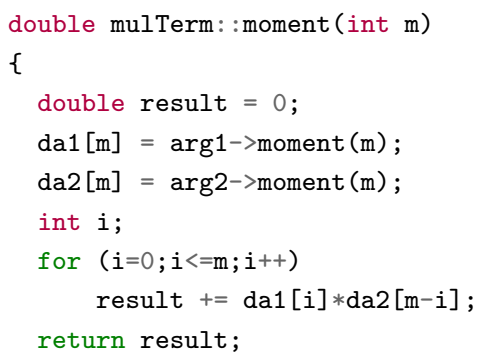

Listing 5.1: Moment function for mulTerm

\subsection{Observations on the C-language code for SPICE device model}

Let us begin by looking at the source code of the SPICE model for a diode, which can be found in Appendix B. The source code begins by evaluating each GENmodel of the diode, which was passed in the DIOload call, as discussed in Section (4.3). It then evaluates each instance of that device model. At the end of the evaluation of each instance, the Jacobian and the RHS of the MNA equations are populated 
directly. This was discussed in section (4.4).

We observe that the source code for the DIOload function, given in Appendix B, mainly consists of a list of assignment operators, which assign a mathematical expression to a variable. We should be able to parse the mathematical expression, and build a tree representing it. If we store all these variable definitions in memory, we should be able to look up the tree associated with any variable. If we do this for the variable $c d$, we find that $c d$ has the definitions shown in listing 5.2 .

As can be seen, there are multiple definitions of the same variable on multiple lines. So which definition should we pick? In the C-language, all lines in the source code are evaluated from top to bottom, in sequential ascending order. So a variable definition on a bigger line number, towards the bottom of the file, will overwrite the definition of the same variable on a previous smaller line number, towards the top of the file. The latest known definition of the variable is it's final definition. Therefore, given a line number in a file, the definition of a variable actively known at that line number, is the definition of the variable at the highest line number smaller than the given line number. If no line number is given, we consider the variable definition at the highest line number in the file. So, in the case of $c d$, the variable definition on line 249 will be chosen as it's starting value.

For example on line 229 of function DIOload, variable evd is defined as evd = $\exp (v d / v t e)$. This definition of evd can be represented by a rooted tree as shown in 


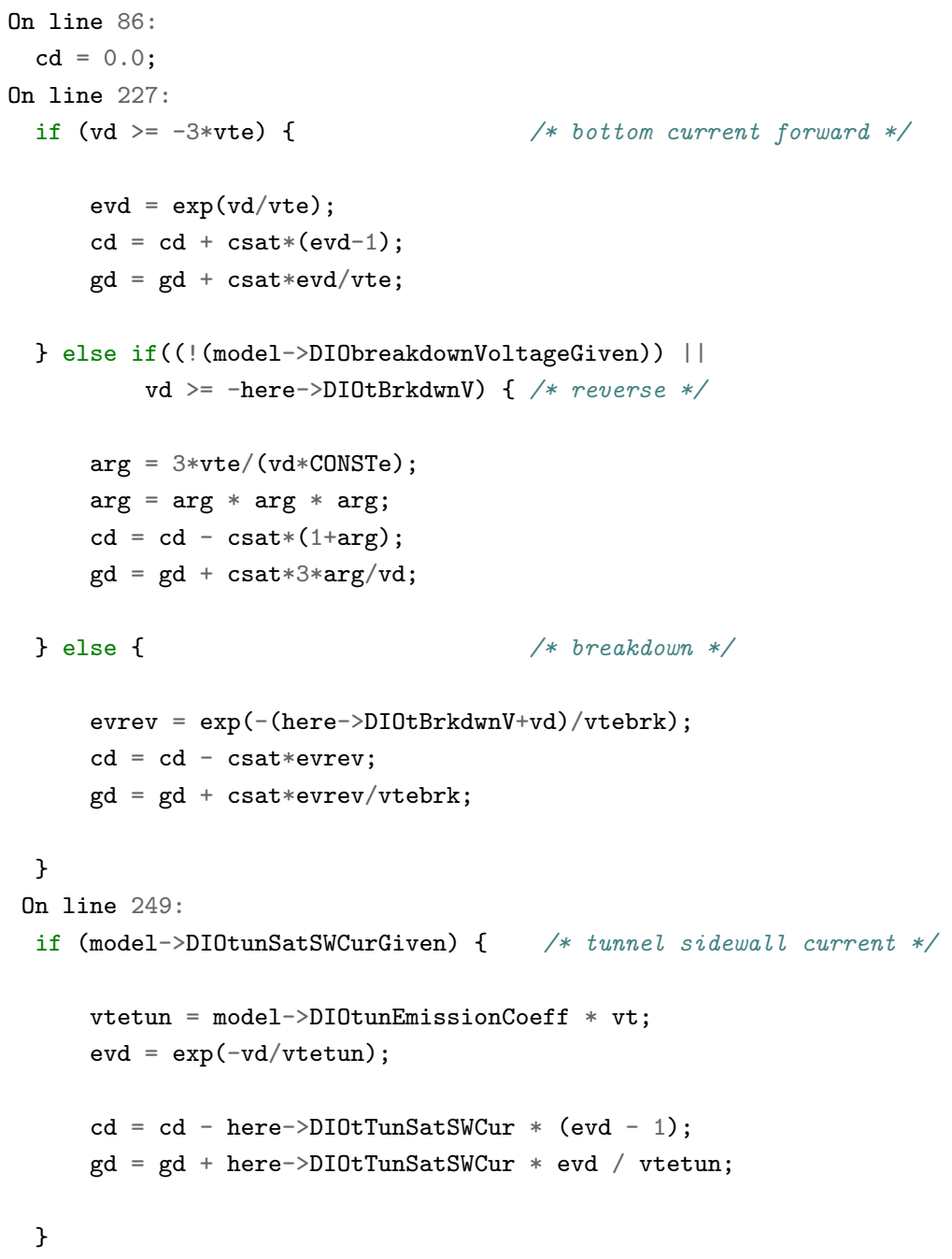

Listing 5.2: Loading of the SPICE diode model in DIOload function

Figure 5.1.

The leaf nodes in this tree are $v d$, vte. But these leaf nodes may not necessarily be constants. For example, variable vte is a leaf node in the tree in Figure (5.1), but it has been defined on line 92 as vte $=$ model->DIOemissionCoeff $f * v t ;$. This can be represented by the tree shown in Figure 5.2 . 


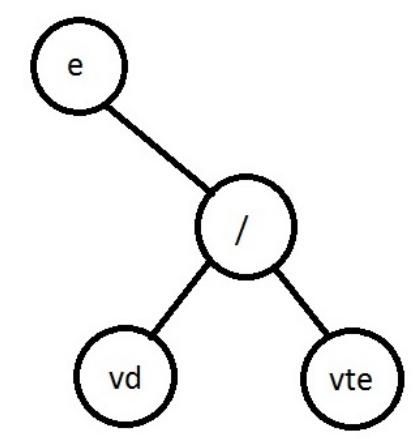

Figure 5.1: Tree for variable evd

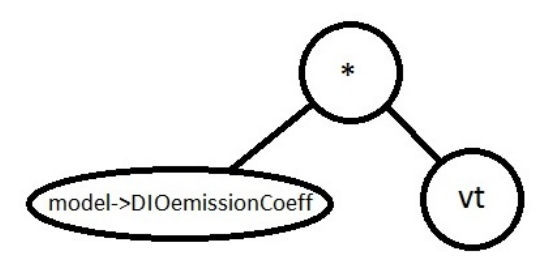

Figure 5.2: Tree for variable vte

Variable $v t$ found in Figure 5.2 is then defined on line 91 as shown in Figure 5.3.

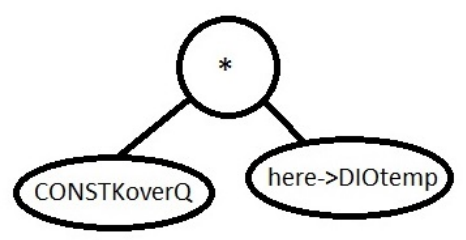

Figure 5.3: Tree for variable $v t$

The leaf nodes of variable $v t$ in Figure (5.3) are CONST KoverQ and here->DIOtemp. Both these terms are constants, and do not have any further definition in the DIOload source code. Hence we can terminate our process of building a rooted tree for variable evd. 
So in the tree definition for variable $e v d$ in Figure (5.1), if we replace variables $v t e$ and $v t$ by their tree representations as shown in Figures (5.2) and (5.3), this leads to a situation like shown in Figure (5.4).

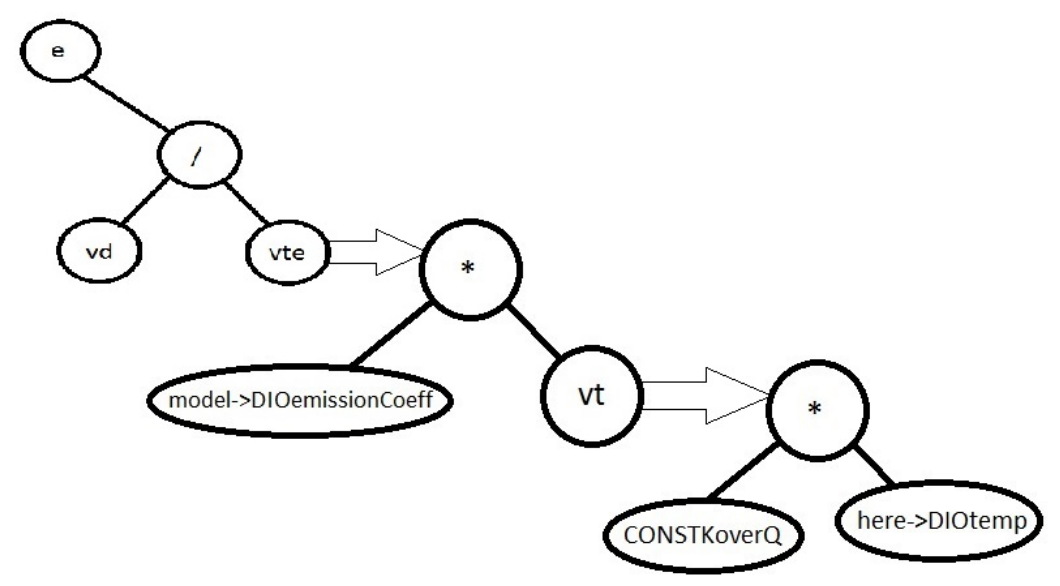

Figure 5.4: Building the Tree for variable $v t$

So all the leaf nodes in a tree should be replaced with the last known definition of the leaf node variables in the file. This will lead to an expanded tree like shown in Figure (5.5).

Thus, when a rooted tree for a variable is requested, if no line number is specified, we have to look up the definition for this variable on the largest line number in the file. We then recursively replace the leaf nodes with their corresponding sub-tree definitions, till no more leaves can be replaced with trees, i.e. all the leaf elements are constants, and do not have any tree definitions in the file. 


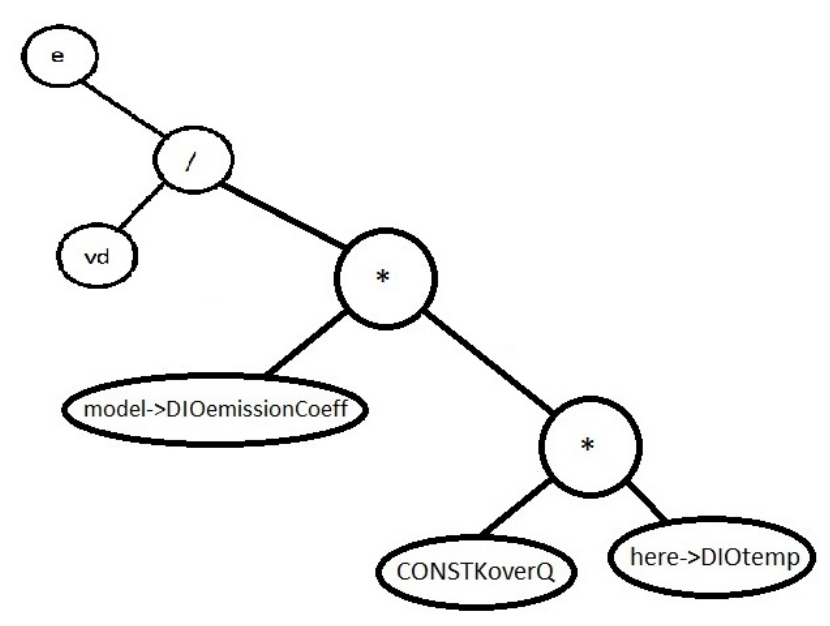

Figure 5.5: Expanded Tree for evd

\subsection{Implementation}

The process of obtaining the rooted tree definition (iExpr) for a variable from the C source code for a SPICE device model, is shown in Figure (5.6). The process begins by converting the $\mathrm{C}$ language source code for the SPICE device model, in to an Abstract Syntax Tree (AST). Source code in any programming language, is inherently hierarchical in nature, and can be very naturally represented as an AST. This process will be described in detail in Section (5.4.1). This process generates an AST for each line in the source code. This AST has a lot of information irrelevant for our purpose of generating rooted tree. So we consider the AST for a line on which a variable is assigned a value, and convert this AST into an internal representation called IVariableDefinition. This process is described in Section (5.4.2). This 
(variable-name, IV ariableDefinition) combination is stored in an internal database called Manager, which is described in Section (5.4.3). As can be seen in Figure (5.6), this process of parsing the $\mathrm{C}$ source code for the device model and loading variable definitions in the Manager happens only once at the beginning of the simulator. This is because, the source code for SPICE device models never change, and it is sufficient to parse this only once.

When the Obreshkov based simulator parses the netlist, it will want to load all the user requested devices in the Jacobian and RHS matrices. At this time, the device loader for the Obreshkov based simulator will call the getIExprVariableDefinition function on the manager, to get the rooted tree definition of a variable on demand. This process involves converting the internal IVariableDefinition for the variable, to the $i$ Expr rooted tree. This process is described in Section (5.4.4).

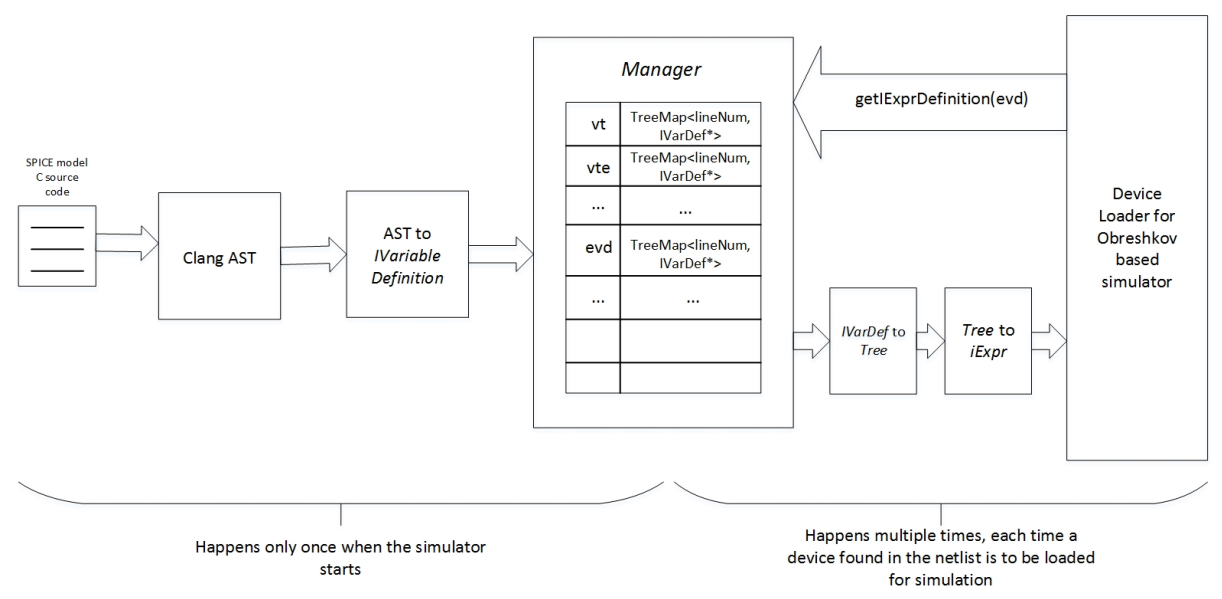

Figure 5.6: Process of obtaining a rooted tree definition for variable, from the SPICE device model code 


\subsubsection{Generating the AST}

As discussed in the previous section, the first step to generate a rooted tree from the source code is to be able to scan for all the assignment operators in the C-source code. For this, we would need to write a C-language parser, that would have knowledge of the grammar for the C-language. This process is somewhat simplified by using a tool called Clang. Clang is a C, C++ language front-end for the LLVM compiler 25].

A compiler for a programming language is a program that takes the source code in that language, and converts it into machine level code, that can be run on the specific processor on the user's machine. There are three main steps involved in the compilation process [25]. These are shown in Figure 5.7.

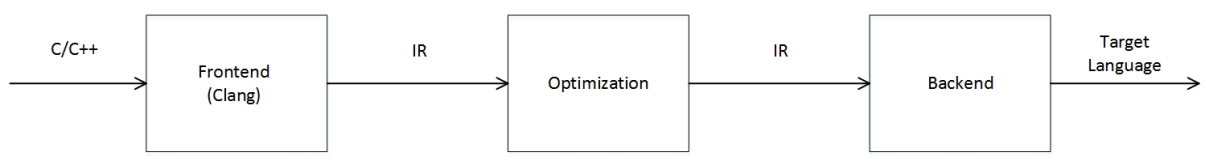

Figure 5.7: Stages during compilation process

A frontend is a part of the compiler, that reads the source code provided by the user, in the source programming language, and converts it into an Abstract Syntax Tree. Every frontend defines it's own Abstract Syntax tree. In the LLVM compiler family, Clang is the frontend for the C-language family. It's job is to convert the C source code to the Clang AST. This AST is then converted into an LLVM Intermediate Representation (IR), which is then passed on to the Optimization and Backend phase, 
for conversion to machine level code. LLVM is responsible for the Optimization and Backend parts. Clang only does the conversion of $\mathrm{C}$ source code to AST, and IR. Consider the $\mathrm{C}$ source code shown in Listing (5.3). The Clang AST for this source code is shown in Listing (5.4).

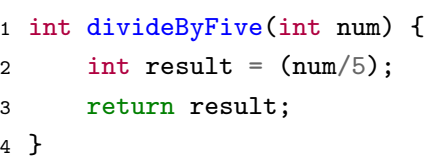

Listing 5.3: Example C code to parse with Clang

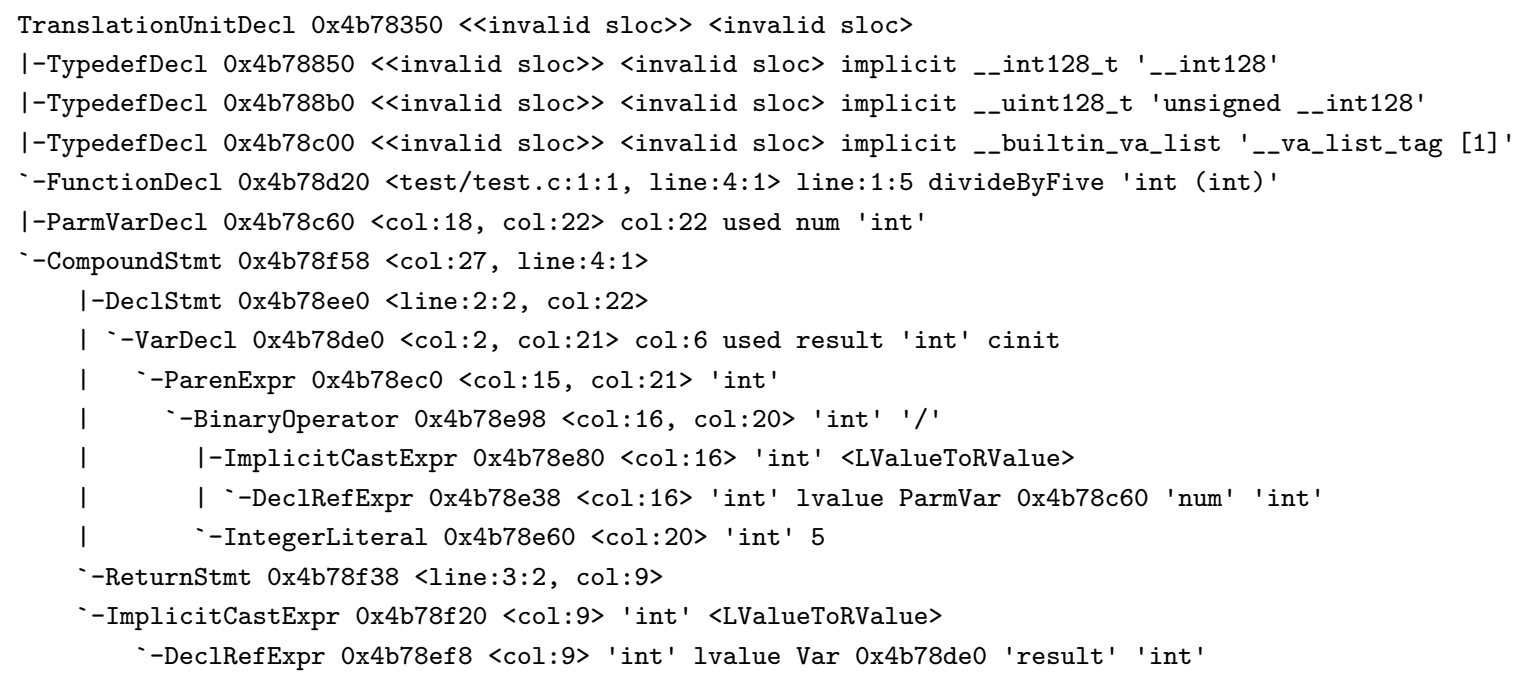

Listing 5.4: Clang AST for C source code in Listing (5.3)

As you can see in Listing (5.4), Clang generates an AST for each line in the source code. For generating a rooted tree for a variable, we are mostly concerned with the lines that have an assignment operator, where a value or an expression on the RHS is assigned to a variable on the LHS. 
An AST can store much more enriched information, than the actual source code. Line numbers are an example. The actual source code does not show the line number. However, while the frontend parses the actual source code, it builds the AST, and adds the extra information of the line number where the node was found, to the AST. This extra information can be of great help for us, while converting this AST into the rooted tree representation that we need.

The Clang AST is different from ASTs of other compilers, in that it very closely represents the $\mathrm{C}$ source code. Most ASTs leave out the details like parenthesis, after parsing. But the Clang AST lets you go all the way to the source code. This also means there is too much detail in the Clang AST, such as the C data type of the variable, for the purpose of using it as the rooted tree.

We will represent our rooted tree with the iExpr base class, as explained in section (5.2). This also allows us the flexibility to replace Clang with some other compiler frontend for the C-language if the need arises.

One of the advantages of using Clang for parsing $\mathrm{C}$ code is that, it has a modular design. This means that all modules of Clang offer a public API, which allows us to include Clang in our own applications. Even though Clang is a frontend for the LLVM compiler family, it can be used as a stand-alone library in our own source code. This stand-alone Clang library is called Libtooling. We will be using the Libtooling library to convert the Clang AST to IVariableDefinition in the next section. 


\subsubsection{Converting the Clang AST to IVariableDefinition}

This is a problem of tree transformation. We treat the Clang AST as the source tree, and the $i$ Expr as the destination tree. In order to decouple the two, we have an internal tree representation called IVariableDefinition. While parsing the C source code, we convert the Clang AST to IVariableDefinition instances, and store them in internal memory.

In the Clang AST, we are interested in only those lines which have an assignment operator, or an If-Statement. Variables are assigned values using an assignment operator in C. These assignment operators can occur inside blocks of If-Statement. In order to look up the correct definition of a variable which is inside an If-Statement block, we need to evaluate the condition of the If-Statement, and choose the appropriate assignment operator based on the success or failure of the condition evaluation.

Thus our internal definition of a variable can be an ordinary tree, which we get from the RHS of an assignment operator, or a conditional definition. This can be modeled with an inheritance hierarchy as shown in Figure (5.8).

\subsubsection{Building Tree from assignment operator}

The tools built with the Clang Libtooling library, run clang:: FrontendActions on the C-source code. This allows the user to define their own custom actions to run on 


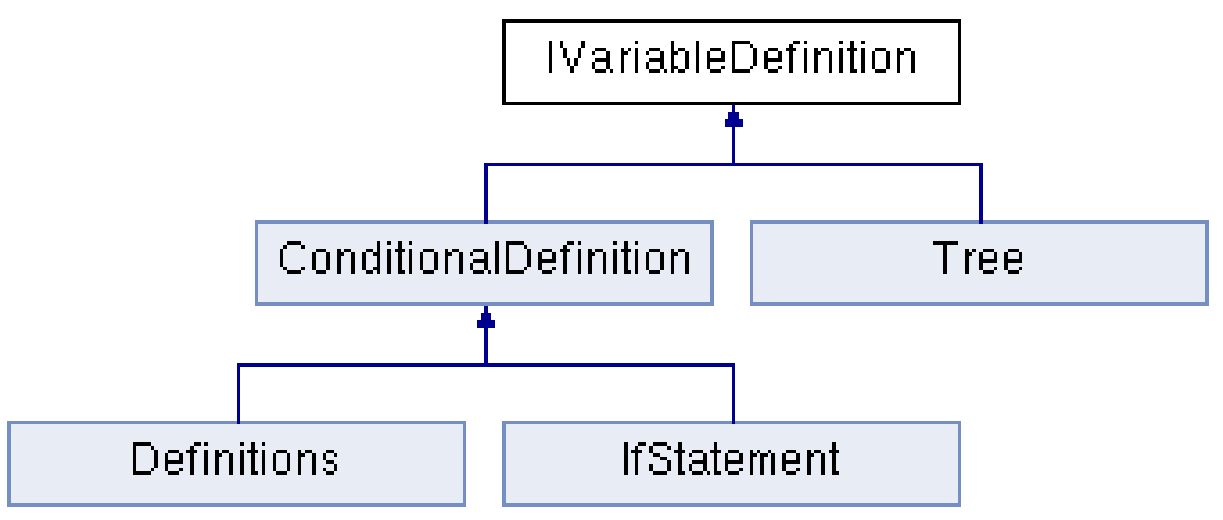

Figure 5.8: Inheritance Diagram for IVariableDefinition

the source code, as part of the compilation process. To run actions over the AST, Clang has provided the clang :: ASTFrontendAction interface, that inherits from clang:: FrontendAction. Since we want to perform our own action on the AST, we will write our own custom action called FindNamedClassAction, which will inherit from the clang :: ASTFrontendAction.

The C source code file that we are trying to parse, is called a TranslationUnit. Thus the topmost element of the Clang AST, as seen in Listing (5.4), is called TranslationUnitDecl. Using this node as the root node, we can recursively traverse the entire Clang AST, and reach every other node we are interested in. This algorithm has been encoded in the clang :: RecursiveASTVisitor class. So, in our FindNamedClassAction, we need to create a new ASTConsumer for our TranslationUnit, which is the dioload.c file for a diode. This ASTConsumer will then create a RecursiveASTVisitor, which will perform pre-order depth first search (DFS) 22 on the AST using TranslationUnitDecl as the root node. 
The RecursiveASTVisitor class provides callback methods, which allow us to write custom actions when certain nodes in the AST are reached. Since we are interested in all the assignment operators in our source code file, we can use the VisitBinaryOperator call back, and inside it, check whether the binary operator is an assignment operator. If we encounter an assignment operator, we can get the RHS from the AST, and perform post-order Depth First Traversal, to convert the RHS AST into our internal Tree representation. This process is shown in the source code in Listing (5.5).

The buildTreeAndGetRoot function, seen in Listing (5.5) is only called on the RHS AST of the assignment operator. In the above code, we take a clang :: Expr pointer as input, because all nodes in the RHS AST derive from the clang::Expr class. If we find that the incoming expression is a BinaryOperator, we create a new Tree :: Node with the data of the binary operator, which could be,$+-{ }^{*}$, or /. Then we recursively call the same buildTreeAndGetRoot function, to convert the left and right sub-trees of the BinaryOperator to Tree objects. Then we return the Tree :: Node.

Usually the leaf elements of a BinaryOperator tree are numbers, such as integers or floats. Whenever such terms are encountered, we create a new Tree :: Node with those numbers as data, and left and right sub-trees as NULL. This terminates the recursive post-order DFS algorithm. 


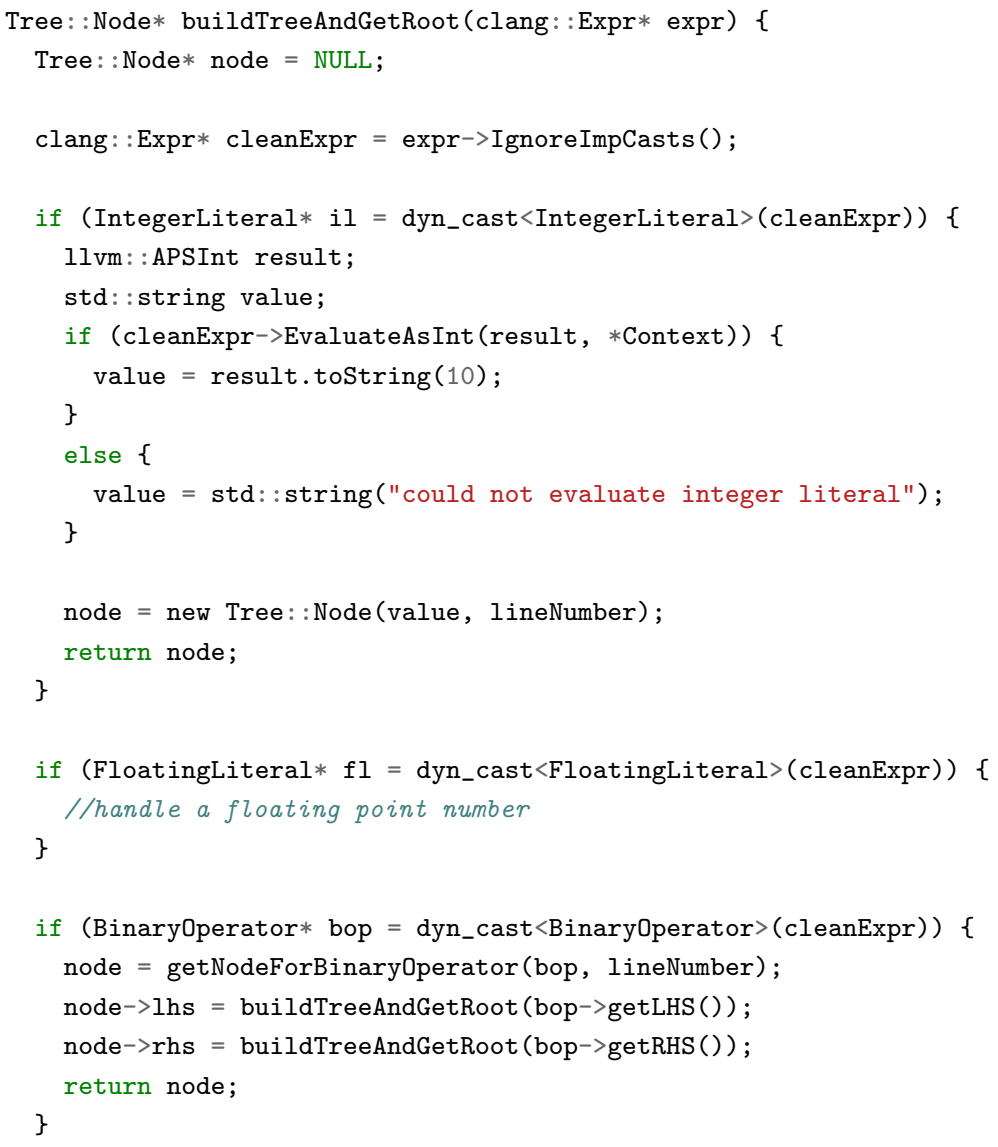

Listing 5.5: Loading of the SPICE diode model in DIOload function

The RHS AST can have several different types of elements, like Unary Operators, Conditional operators etc. These can all be handled by dynamic-casting the input clang :: Expr to any of these operators, and providing code to handle them.

Thus the RHS AST of an assignment operator is converted to the internal Tree representation. The LHS AST contains the name of the variable being defined. We then insert this pair of (variable-name, Tree-definition) objects in our manager 
database, as explained in the next section.

\subsubsection{Building ConditionalDefinition from If-Statement}

The process of generating a ConditionalDefinition is similar to the process of generating a Tree described above. In the clang:: RecursiveASTVisitor, it is possible to determine if the statement on the current line is an If-Statement or not. Whenever an clang :: If Statement is encountered in the AST, the ASTVisitor calls the buildTreeAndGetRoot function of Listing (5.5) on the condition of the If-Statement, the success and fail blocks. For each If-Statement that is encountered in the AST, a new ConditionalDefinition object is created, with the appropriate condition, success and fail blocks that were extracted from the AST, and is passed to the Manager for storage. The Manager maintains a pointer to the current-if-statement. If a new variable definition or if-statement is encountered, then it's line number is first checked with the current-if-statement. If the variable definition lies inside the if-statement, it is inserted in the ConditionalDefinition for that if-statement, and a pointer to that ConditionalDefinition is inserted in the Manager described in the next section.

\subsubsection{Storing the internal IVariableDefinition in memory}

Once the IVariableDefinition for a variable has been found, it needs to be stored in internal memory, so that it can be easily looked up on demand, when generating 
the rooted tree $i$ Expr for a variable.

This can be done by using a HashMap, where the variable name is used as the key, and the internal Tree representation is used as the value. This will allow us to look up the Tree definition for any variable in constant time 22$]$.

However, there can be multiple IV ariableDefinition-s for each variable, and given a variable name and a line number, we need to find a IVariableDefinition for the variable which is at the highest line number less than the input line number. This means that we need to store all the IVariableDefinition-s of the same variable, in a ordered collection. This can be done by using a TreeMap [22], that allows us to look up the highest line number less than a given line number in $\log (n)$ time, where $n$ is the number of Tree-definitions of the variable. The key in the TreeMap will be the line number, and value will be the IVariableDefinition.

Thus given a variable name, and a line number where the variable is being used, the last known definition of that variable can be found in $\log (n)$ time, where $n$ is the number of definitions of that variable in the file. We call this data structure the

\section{Manager.}

It must be noted that parsing of the $\mathrm{C}$ source code, and populating the Manager, only needs to be performed once, when the simulator is being started. The definition of the SPICE device models never change, and the definitions of the variables in the SPICE models can be stored in memory. During the run of the simulator, when a 
netlist is being read, if a nonlinear device like a diode is requested in the netlist, we need to know the rooted tree definition of the variable that represents the nonlinear current. This was discussed in Section 4.3.1). If the variable that represents this nonlinear current in the DEVload source code for that device is known, then we ask our Manager to give us the definition of this variable that represents the nonlinear current in the $i$ Expr format. These queries will be performed for the number of times a SPICE nonlinear device is requested in the netlist. This process of on-demand conversion of internal Tree representation to $i$ Expr, is explained in the next section.

\subsubsection{Converting IVariableDefinition to $i$ Expr}

The iExpr definition of a variable can be retrieved using the method getIExprVariableDefinition invoked on the Manager. This method is implemented in a two step

process. The first step involves looking up the last known definition of the requested variable in the database, and then expanding this Tree, till all the variables have been replaced by constant leaf nodes. The second step involves converting the expanded internal Tree to the iExpr definition.

\subsubsection{Building the expanded internal Tree representation}

This process performs two important operations. The first operation is to look up the last known definition of a variable or leaf nodes. The second operation is that, if 
the last known definition of a variable happens to be a ConditionalDefinition, then this If-Statement is evaluated, and the appropriate Tree for the variable is chosen. This process ensures that all the If-Statements in a variable definition are simplified and replaced with a single assignment operator Tree definition. This process is represented by the Algorithm (5.1, 5.2, 5.3). The structure for the Tree class is shown in Listing (5.6).

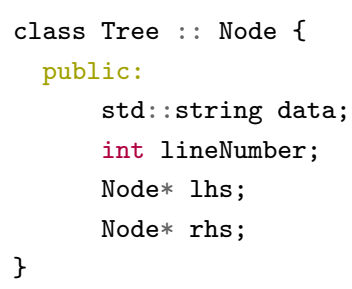

Listing 5.6: Tree Data Structure

\subsubsection{Converting the expanded internal Tree to $i$ Expr}

This process involves doing depth first traversal on the entire expanded internal Tree, and for each node in the Tree, build a new iExpr node that is appropriate for the data in the Tree node. So, when a '+' operator is encountered in the Tree node, a new addTerm is created in the iExpr tree. For a 'e' term in the Tree node, a new expTerm is created in the $i$ Expr tree. Each type of term in the $i$ Expr tree, knows 


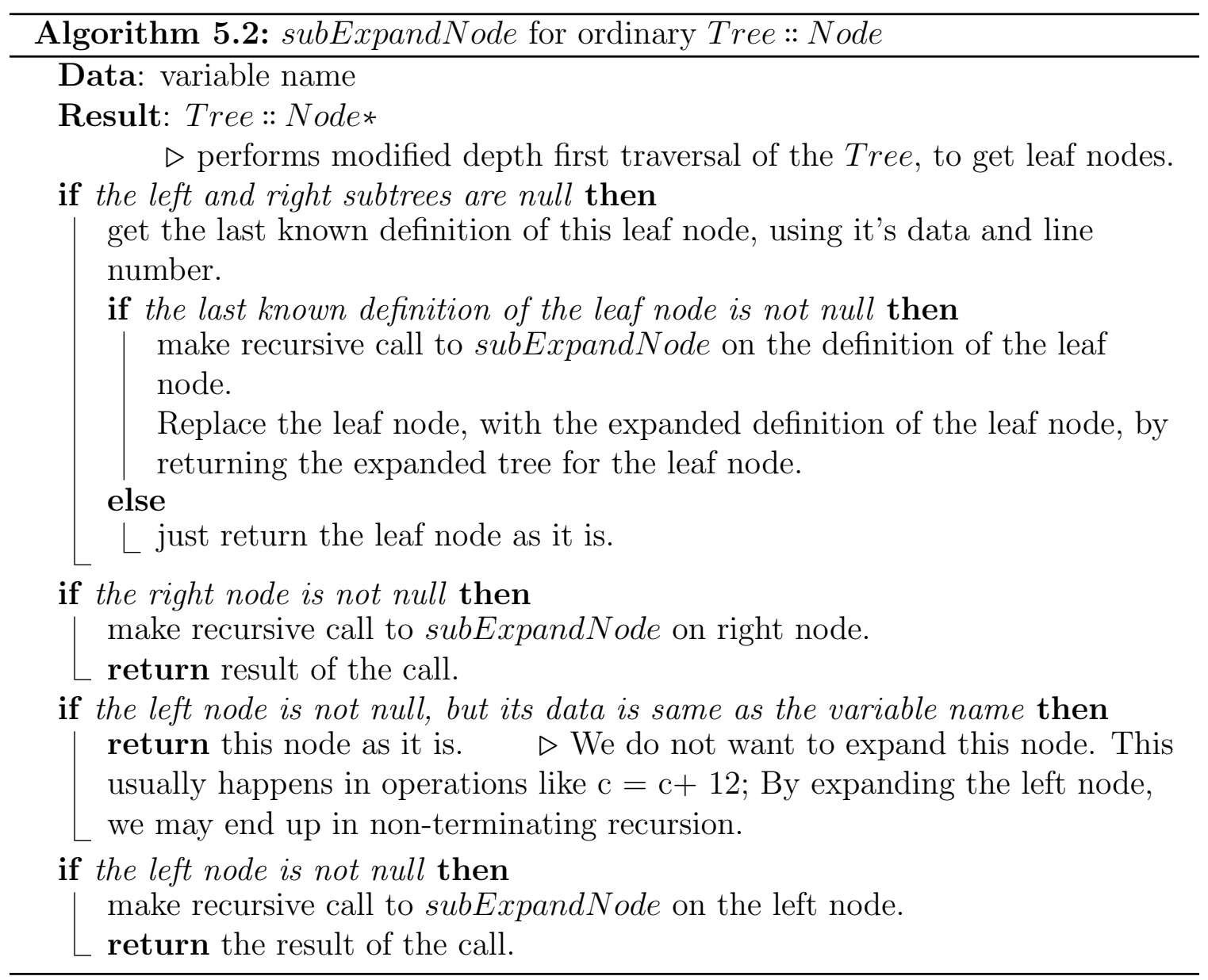

how to best calculate it's moments. Hence it is important to create the proper $i$ Expr node based on the data in the Tree node. This process is shown in Algorithm 5.4 .

For CondNode, we call convertTreeToIExpr recursively on the condition, left and right elements of the Tree :: CondNode, and return the new condTerm that was created.

The SPICE device models use several relational and logical operators in their code, to load the model. So it was necessary to make sure that corresponding operator nodes 


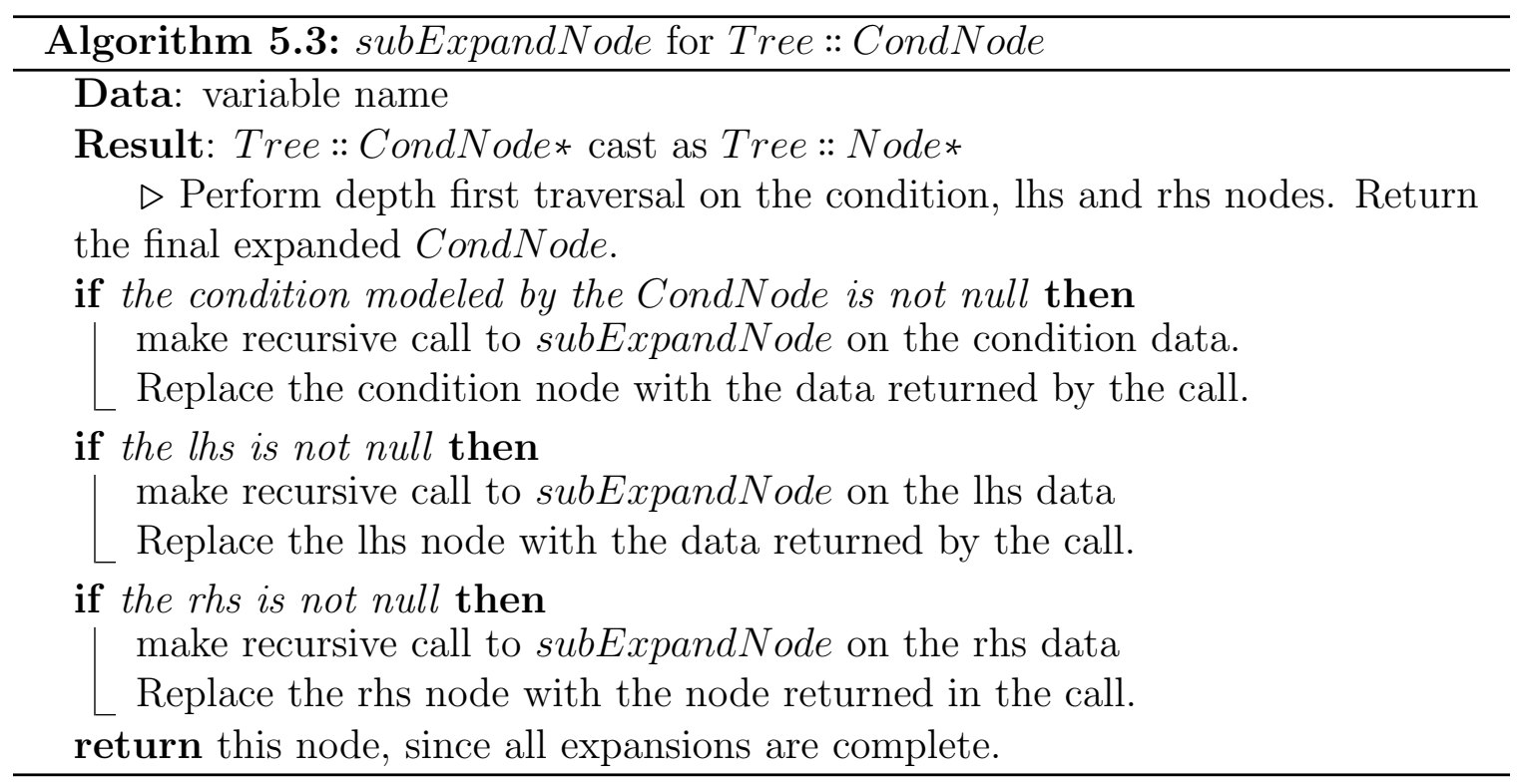

were available in the $i$ Expr nodes. Hence it was necessary to define relationalTerm and logicalTerm nodes in the iExpr, to find the proper moments when these nodes are encountered in the circuit.

\subsection{Discussion}

This chapter discussed how the $\mathrm{C}$ source code for the SPICE device model can be used to build a rooted tree representation of the nonlinear currents of the device. Using this parsing technique, the resistive contribution of the device and the rooted tree for the nonlinear current can be extracted, and integrated in the Obreshkov based circuit simulation using a new element called SPICE_DIODE. The next section will show the results of using a SPICE_DIODE in an actual circuit and will compare its 


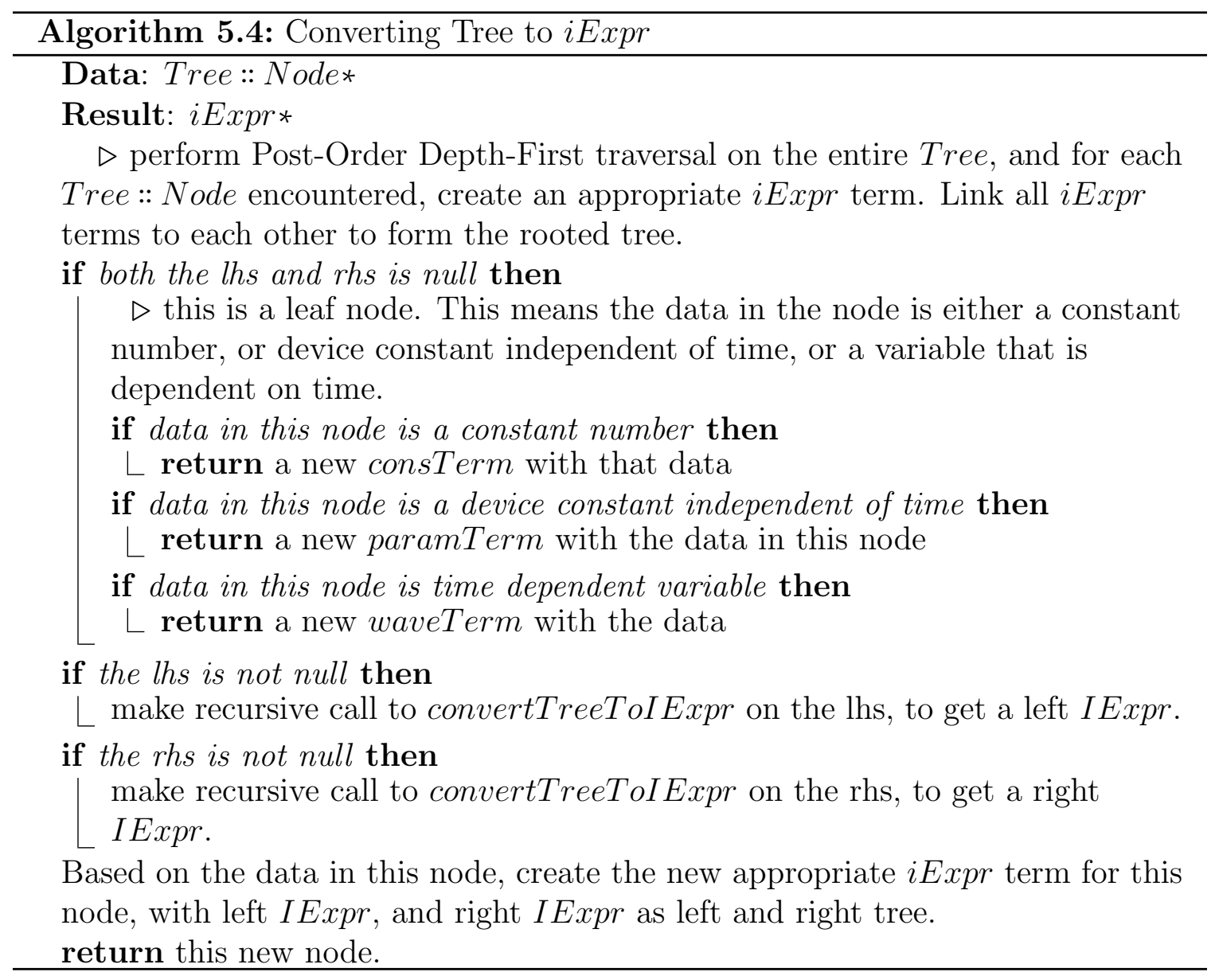

results with the same circuit simulated using NGSPICE. 


\section{Chapter 6}

\section{Numerical Results}

\subsection{Results}

In order to test the framework developed to integrate Generic SPICE models in the Obreshkov based method, a half wave peak rectifier circuit was simulated using the Obreshkov based method and with HSPICE. The peak rectifier circuit is shown below in Figure 6.1.

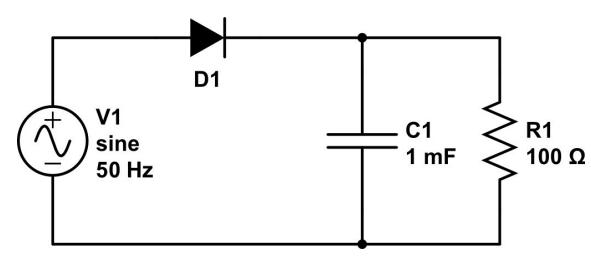

Figure 6.1: Peak Rectifier Circuit

While simulating with the Obreshkov formula, the $\mathrm{C}$ code for the SPICE diode model is parsed using the framework developed in Chapter 5. The nonlinear current 
of the diode is represented by a rooted tree, for calculating higher order derivatives. This rooted tree is shown in Listing 6.1.

The terms $x(1)$ and $x(2)$ in the rooted tree, are the voltages across the nonlinear current of the diode. The terms beginning with model->, here->, or ckt->, are constants used in the rooted tree. The constants beginning with model-> are the model specific constants of the device, those beginning with here-> are the instance specific constants, and those beginning with $c k t->$ are the global constants used in the CKT circuit structure. Typically, circuit simulators have predefined values of these constants, stored as default model and instance parameters. The user is given the freedom to override these constants for their particular type of device to be used in the simulation. The user overrides these constants by specifying custom models or instances of the SPICE device in the netlist.

\subsection{Extracting values of the constants required in the rooted tree}

In order to evaluate a rooted tree, we need to know the values of the constants appearing in the rooted tree. For the purpose of this study, we have used the default values of these constants, which are available in NGSPICE as default model and instance parameters. This has two main advantages. The first advantage is that, we 
((0+if ( if $(\{["$ ckt->CKTmode" $]=544\}$ \& 2048) then $\{\{[" *$ (ckt->CKTstates $[0]+$ here->DIOstate)"]=0.730268\} \} else \{ if ( $\{[$ "ckt->CKTmode"]=544\} \& 4096) then \{

$\{[" *($ ckt->CKTstates $[1]+$ here->DIOstate $) "]=0\}\}$ else $\{$ if $(\{["$ ckt->CKTmode"] $=544\} \& 512 \& \&$

$\{$ ["ckt->CKTmode"]=544\} \& 32 \&\& $\{[$ "ckt->CKTmode"]=544\} \& 65536) then $\{$ ["here->DI0initCond"]=0 $\}$ \}

else $\{$ if $(\{["$ ckt->CKTmode" $]=544\}$ \& 512 \&\& $\{[$ "here->DIOoff"]=0\}) then $\{0\}$ else $\{$ if (

$\{["$ ckt->CKTmode"] $=544\} \& 512)$ then $\{\{[$ "here->DIOtVcrit"]=0.730268\} $\}$ else $\{$ if (

$\{["$ ckt->CKTmode"] $=544\} \& 1024 \& \&\{[" h e r e->D I O o f f "]=0\})$ then $\{0\}$ else $\{(x(1)-x(2))\}\}$

\}$>=-1 * 3 *\{[$ model->DIOemissionCoeff"] =1\}*\{["CONSTKoverQ"] =8.61709e-05\}*\{["here->DI0temp"]=300.15\})

then $\{\{["$ here->DIOtSatCur"]=1e-14\}*(exp(if ( \{["ckt->CKTmode"]=544\} \& 2048) then \{

$\{[" *($ ckt $->C K T s t a t e s[0]+$ here->DIOstate)"] $=0.730268\}$ \} else $\{$ if ( \{["ckt->CKTmode"]=544\} \& 4096)

then $\{\{[" *($ ckt->CKTstates $[1]+$ here->DIOstate)"] $=0\}\}$ else $\{$ if ( $\{["$ ckt->CKTmode"]=544\} \& 512 \&\&

$\{["$ ckt->CKTmode"] =544\} \& 32 \&\& $\{[$ "ckt->CKTmode"]=544\} \& 65536) then $\{$ ["here->DIOinitCond"]=0\} \}

else $\{$ if $(\{["$ ckt->CKTmode"] $=544\}$ \& 512 \&\& $\{[$ "here->DIOoff"]=0\}) then $\{0\}$ else $\{$ if (

$\{["$ ckt->CKTmode"] $=544\}$ \& 512) then $\{\{[$ "here->DIOtVcrit"]=0.730268\} $\}$ else $\{$ if (

$\{[$ ckt->CKTmode"]=544\} \& 1024 \&\& $\{[$ "here->DIOoff"] $=0\})$ then $\{0\}$ else $\{(x(1)-x(2))\}\}\}$

\}$/\{[$ model->DIOemissionCoeff"] =1\}*\{["CONSTKoverQ"] =8.61709e-05\}*\{["here->DIOtemp"] =300.15\})-1) $\}$

else $\{$ if ( $\{$ ["model->DIObreakdownVoltageGiven"]=0\} || if ( $\{[$ "ckt->CKTmode"]=544\} \& 2048) then \{

$\{[" *($ ckt->CKTstates $[0]+$ here->DIOstate)"]=0.730268\} \} else \{ if ( \{["ckt->CKTmode"]=544\} \& 4096)

then $\{\{[" *($ ckt->CKTstates $[1]+$ here->DIOstate)" $]=0\}\}$ else $\{$ if ( $\{["$ ckt->CKTmode"]=544\} \& 512 \&\&

$\{["$ ckt->CKTmode"] $=544\}$ \& 32 \&\& $\{["$ ckt->CKTmode"]=544\} \& 65536) then $\{$ ["here->DIOinitCond"]=0\} \}

else $\{$ if $(\{["$ ckt->CKTmode"] $=544\}$ \& 512 \&\& $\{[$ "here->DIOoff"]=0\}) then $\{0\}$ else $\{$ if (

$\{["$ ckt->CKTmode"] $=544\}$ \& 512) then $\{\{[$ "here->DIOtVcrit"]=0.730268\} $\}$ else $\{$ if (

$\{["$ ckt->CKTmode"] $=544\} \& 1024 \& \&\{["$ here->DIOoff"]=0\}) then $\{0\}$ else $\{(x(1)-x(2))\} \quad\} \quad\} \quad\}$

\}$>=-1 *\{[$ "here->DIOtBrkdwnV"]=0\}) then $\{\{[$ "here->DIOtSatCur"]=1e-14\}*(1+3*\{["model->DIOemissionC

oeff"] $=1\} *\{[$ "CONSTKoverQ"] =8.61709e-05\}*\{["here->DIOtemp"]=300.15\}/if ( $\{$ ["ckt->CKTmode"]=544\} \&

2048) then $\{\{[" *($ ckt->CKTstates $[0]+$ here->DIOstate)" $]=0.730268\}$ else $\{$ if (

$\{["$ ckt->CKTmode"] $=544\} \&$ 4096) then $\{\{[" *($ ckt->CKTstates $[1]+$ here->DIOstate)"]=0\} $\}$ else $\{$ if ( $\{["$ ckt->CKTmode"] $=544\}$ \& 512 \&\& $\{["$ ckt->CKTmode"] $=544\}$ \& 32 \&\& \{["ckt->CKTmode"]=544\} \& 65536) then $\{\{[" h e r e->D I D i n i t C o n d "]=0\}\}$ else $\{$ if ( $\{$ ["ckt->CKTmode"] $=544\}$ \& 512 \&\& $\{[$ "here->DIOoff"]=0\}) then $\{0\}$ else $\{$ if ( $\{["$ ckt->CKTmode"] $=544\}$ \& 512) then $\{\{[" h e r e->D I 0 t V c r i t "]=0.730268\}\}$ else \{ if ( $\{["$ ckt->CKTmode"] $=544\} \& 1024 \& \&\{["$ here->DIOoff"]=0\}) then $\{0\}$ else $\{(x(1)-x(2))\}\}$ \}$\}\} *\{["$ CONSTe"] $=2.71828\} * 3 *\{[$ [model->DIOemissionCoeff"] $=1\} *\{[$ "CONSTKoverQ"] $=8.61709 e-05\} *\{["$ her e->DIOtemp"]=300.15\}/if ( $\{[$ "ckt->CKTmode"]=544\} \& 2048) then $\{\{[" *($ ckt->CKTstates $[0]+$ here->DIOstate)"]=0.730268\} $\}$ else $\{$ if $(\{["$ ckt->CKTmode"] $=544\} \& 4096)$ then \{

$\{[" *($ ckt->CKTstates [1] + here->DIOstate)"]=0\} $\}$ else $\{$ if ( $\{$ ["ckt->CKTmode"]=544\} \& 512 \&\&

$\{["$ ckt->CKTmode"] $=544\}$ \& 32 \&\& $\{["$ ckt->CKTmode"] =544\} \& 65536) then \{ \{["here->DIOinitCond"]=0\} \}

else $\{$ if $(\{["$ ckt->CKTmode"] $=544\}$ \& 512 \&\& $\{["$ here->DIOoff"]=0\}) then $\{0\}$ else $\{$ if (

$\{["$ ckt->CKTmode"] $=544\} \&$ \& 512) then $\{\{$ ["here->DIOtVcrit"] $=0.730268\}\}$ else $\{$ if (

$\{["$ ckt->CKTmode"] $=544\} \& 1024 \& \&\{[$ "here->DIOoff"]=0\}) then $\{0\}$ else $\{(x(1)-x(2))\}\}$ \}$*\{["$ CONSTe"] $=2.71828\} * 3 *\{$ ["model->DIOemissionCoeff"] $=1\} *\{[$ "CONSTKoverQ"] $=8.61709 e-05\} *\{["$ here->DIO temp"]=300.15\}/if $(\{["$ ckt->CKTmode" $]=544\} \&$ 2048) then $\{\{[" *($ ckt->CKTstates $[0]+$ here->DIOstate)"]=0.730268\} $\}$ else $\{$ if ( $\{$ ["ckt->CKTmode"] $=544\}$ \& 4096) then \{ $\{[" *($ ckt->CKTstates $[1]+$ here->DIOstate)"]=0\} $\}$ else $\{$ if ( $\{$ ["ckt->CKTmode"]=544\} \& $512 \& \&$ $\{[$ "ckt->CKTmode"] =544\} \& 32 \&\& $\{$ ["ckt->CKTmode"]=544\} \& 65536) then $\{$ ["here->DIOinitCond"]=0\} $\}$ else $\{$ if $(\{["$ ckt->CKTmode"] $=544\}$ \& 512 \&\& $\{[$ "here->DIOoff"]=0\}) then $\{0$ \} else $\{$ if ( $\{["$ ckt->CKTmode"]=544\} \& 512) then $\{$ ["here->DIOtVcrit"]=0.730268\} $\}$ else $\{$ if (

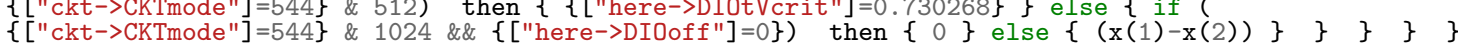

\}$*\{["$ CONSTe"] $=2.71828\})\}$ else $\{\{[$ "here->DIOtSatCur"] $=1 e-14\} * \exp (-1 *(\{["$ here->DIOtBrkdwnV"] $=0\}+i f$

$(\{["$ ckt->CKTmode"] $=544\} \&$ 2048) then $\{\{[" *($ ckt->CKTstates $[0]+$ here->DIOstate $) "]=0.730268\}\}$ else $\{$ if $(\{["$ ckt->CKTmode" $]=544\}$ \& 4096) then $\{\{[" *($ ckt->CKTstates $[1]+$ here->DIOstate $) "]=0\}\}$ else \{ if $(\{["$ ckt->CKTmode"] $=544\}$ \& 512 \&\& $\{$ ["ckt->CKTmode"] =544\} \& 32 \&\& \{["ckt->CKTmode"]=544\} \& 65536) then $\{\{["$ here->DIOinitCond"]=0\} $\}$ else $\{$ if $(\{["$ ckt->CKTmode"] $=544\}$ \& 512 \&\& $\{[" h e r e->D I O o f f "]=0\})$

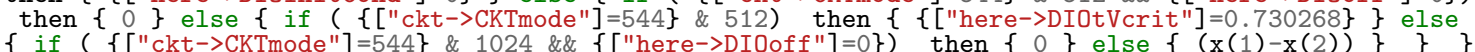
$\left\{\right.$ if $_{\}}(\{[$"ckt->CKTmode"] $=544\}$ \& 1024 \&\& $\{[$ "here->DIOoff"]=0\}) then $\{0\}$ else $\{(x(1)-x(2))\}\}$ )$/\{[$ "model->DIObrkdEmissionCoeff"]=1\}*\{["CONSTKoverQ"]=8.61709e-05\}*\{["here->DI0temp"]=300.15\}) $\} \quad\}$ ) if $(\{[$ model->DIOtunSatSWCurGiven"] $=0\})$ then $\{\{[$ "here->DIOtTunSatSWCur"] $=0\} *(\exp (-1 * i f($ $\{["$ ckt->CKTmode"] $=544\} \&$ 2048) then $\{\{[" *($ ckt->CKTstates $[0]+$ here->DIOstate $) "]=0.730268\}\}$ else \{

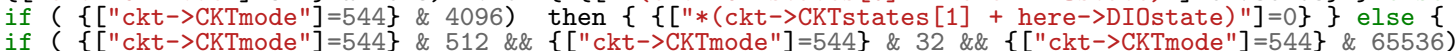
then $\{\{[" h e r e->D I O i n i t C o n d "]=0\}\}$ else $\{$ if ( $\{["$ ckt->CKTmode"]=544\} \& 512 \&\& \{["here->DIOoff"]=0\}) then $\{0\}$ else $\{$ if $(\{["$ ckt->CKTmode"]=544\} \& 512) then $\{$ \{["here->DIOtVcrit"]=0.730268\} $\}$ else $\{$ if ( $\{[$ "ckt->CKTmode"] $=544\} \& 1024$ \&\& $\{$ ["here->DIOoff"]=0\}) then $\{0\}$ else $\{(x(1)-x(2))\}\}$ \}$\}$ $/\{[$ model->DIOtunEmissionCoeff"] =30\}*\{["CONSTKoverQ"]=8.61709e-05\}*\{["here->DI0temp"]=300.15\})-1) $\}$ else $\{0\}$ )

Listing 6.1: Rooted Tree for nonlinear current of the diode 
have an assurance that the values of these constants is the same as that used in the NGSPICE simulator, and we are not relying on our interpretation of these constants. The second is that all the constants are readily available in NGSPICE, and we do not have to resort to external data sheets to find out the values of each constant.

In order to extract the values of these constants, we ran the NGSPICE circuit simulator in Debug mode using the GDB debugger in Linux, and used a Python script to extract, in bulk, values of all the constants required to evaluate the rooted tree. The Python script takes as input, a list of all the constants required to evaluate the rooted tree, and produces as output, a HashMap [22] of all the constants with their values. The Python script automates the process of setting up breakpoints in the SPICE device model source code where the values of these constants are available. When the breakpoints are hit with GDB, it extracts the values of all the constants provided to it as input, in a HashMap.

The constants required to evaluate a rooted tree, appear only in the leaf elements of the rooted tree. So the list of constants, which the Python script requires as input, can be generated by building a list of the data contained in the leaves of the rooted tree. This can be done by performing a Depth First Search [22] on the rooted tree with the objective of finding all the leaves, and reading their data to build the list. Once all the constant values are known, the rooted tree for the nonlinear current of the diode can be evaluated. 
The half wave peak rectifier of Figure (6.1) was simulated using the Obreshkov method with order $(\mathrm{m}, \mathrm{n})$ of $(5,5)$. These results were compared to the simulation output from HSPICE. This comparison is shown in Figure 6.2). It can be seen that these results agree closely with each other.

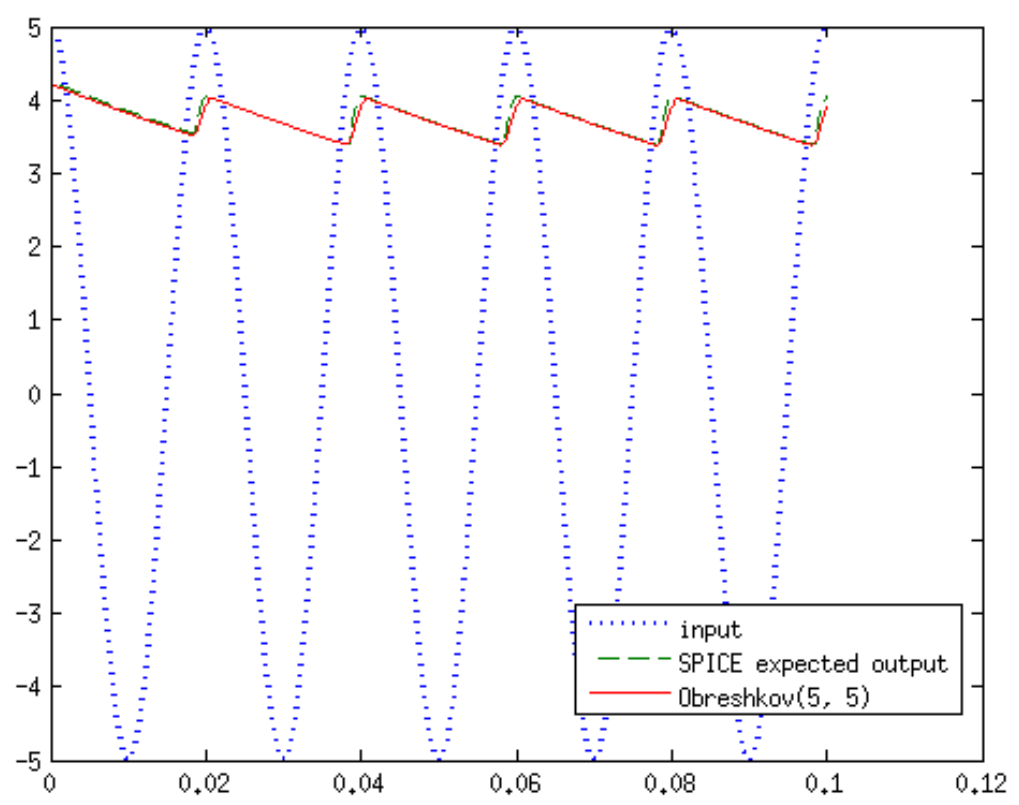

Figure 6.2: Comparison of Peak Rectifier output of Obreshkov method of order (5, 5) with HSPICE

\subsection{Evaluating derivatives of the rooted tree}

Consider the Equation 4.2 for nonlinear current of the diode. If we represent the nonlinear diode current $c d$ by $i$, and assume the convergence aid $c k t->C K T g m i n$ is 0 , then first order derivative of the diode current $i$ with respect to time is given by 


\begin{tabular}{|ccc|}
\hline moment & expected & rooted-tree-moment \\
\hline 0 & 0.271064 & 0.271064 \\
1 & 52.401338 & 52.401338 \\
2 & 5065.05 & 5065.05 \\
3 & 326387 & 326387 \\
\hline
\end{tabular}

Table 6.1: Comparison of hand calculated derivatives of the rooted tree for the diode current, with the result of the moment function invoked on the rooted tree

Equation 6.1.

$$
\begin{array}{r}
\frac{d i}{d t}=\frac{\partial i}{\partial v_{p}} \frac{d v_{p}}{d t}+\frac{\partial i}{v_{n}} \frac{d v_{n}}{d t} \\
\frac{d i}{d t}=\left[\left(\frac{I_{s}}{n V_{t}} e^{\frac{v_{p}-v_{n}}{n V_{T}}}\right) \frac{d v_{p}}{d t}\right]+\left[-\left(\frac{I_{s}}{n V_{t}} e^{\frac{v_{p}-v_{n}}{n V_{T}}}\right) \frac{d v_{n}}{d t}\right]
\end{array}
$$

Using the constants $I_{s}=1 \times 10^{-14} \mathrm{~A}, n V_{T}=25.9 \times 10^{-3} \mathrm{~V}, v_{p}=1.8 \mathrm{~V}, v_{n}=1 \mathrm{~V}, \frac{d v_{p}}{d t}=9$ $\mathrm{V} / \mathrm{s}, \frac{d v_{n}}{d t}=4 \mathrm{~V} / \mathrm{s}$, the diode current $i$ evaluates to $0.271064 \mathrm{~A}$, and the derivative of $i$ with time $\frac{d i}{d t}$ evaluates to $52.4013 \mathrm{~A} / \mathrm{s}$. Using the same constants, the derivatives of the rooted tree for the nonlinear diode current $c d$, obtained from the SPICE device model were calculated using the moment function of $i$ Expr* discussed in Section (5.2). These hand calculated derivatives of the diode current $i$, were compared to those calculated for the rooted tree. The results are shown in Table 6.1.

Thus we can see that the hand calculated derivatives of the rooted tree for the diode current, match those computed from the moment function invoked on the rooted tree, for up to 6 decimal places. 


\section{Chapter 7}

\section{Conclusions and Future Work}

\subsection{Conclusions}

In this thesis, a framework was developed to integrate Generic SPICE device models in the Obreshkov based high-order simulation method. This was done in two steps.

In the first step, a technique was developed to parse the C language based source code for SPICE device models, and to exploit a common pattern of companion models to identify the the nonlinear current, conductive and capacitive effects of the device model. This framework can identify the details in the generic SPICE device models relevant to the Obreshkov based simulation method, and can ignore artifacts in the source code that are dependent on the integration method used in SPICE, and the first order derivate of the nonlinear current with respect to voltage used in the NewtonRaphson method. 
In the second step, a rooted tree was generated for the nonlinear current variable, which was identified in the $\mathrm{C}$ source code, using the technique developed in the first step. A framework was developed to get the rooted tree for nonlinear currents of SPICE device models on demand, as required in the simulation, and to load the Jacobian matrix $\tilde{\boldsymbol{J}}$ of Equation 3.10 with the high-order derivatives required for the Obreshkov based method. This framework was tested using the SPICE device model for a diode, in a peak rectifier circuit, and its results were found to closely match the results obtained from simulation in SPICE.

\subsection{Future Work}

Electronic devices are characterized by their nonlinear current, resistive and capacitive behaviour. Generic SPICE device models represent this behaviour using companion models for nonlinear current and capacitive behaviour, as seen in Section (2.6). This thesis has developed a way to parse the $\mathrm{C}$ source code for generic SPICE device models, extract the nonlinear current, capacitive and resistive contribution of the device, and load these contributions in the Obreshkov based Jacobian matrix $\tilde{\boldsymbol{J}}$, shown in Equation (3.10) and RHS vector. However some future work remains, which is enlisted below:

- As mentioned in Chapter 4, the capacitive effect of SPICE device 
models needs to be integrated in the Obreshkov based method. The framework developed in Chapter 5, has the capacity to detect the occurrence of the companion form of the capacitor occurring in the SPICE device model source code. However, for generic nonlinear devices, the depletion region around the p-n junction behaves as a nonlinear capacitor. Thus more work will need to be done to integrate this nonlinear capacitor in the Obreshkov based Jacobian matrix $\tilde{\boldsymbol{J}}$, and RHS vector. This can be done along the lines of the work published in [24].

- When testing a Bridge rectifier circuit in the Obreshkov based simulation method, using the SPICE_DIODE model parsed from the C source code, the algorithm was not able to converge. This needs to be further investigated by providing appropriate initial conditions, and testing with higher orders of the Obreshkov based method.

- The time taken by the Obreshkov based method to simulate a circuit, needs to be compared with the time taken by the traditional SPICE simulators using the generic SPICE device models. 


\section{Appendices}




\section{Appendix A}

\section{Source code for $C K T$ circuit struct}

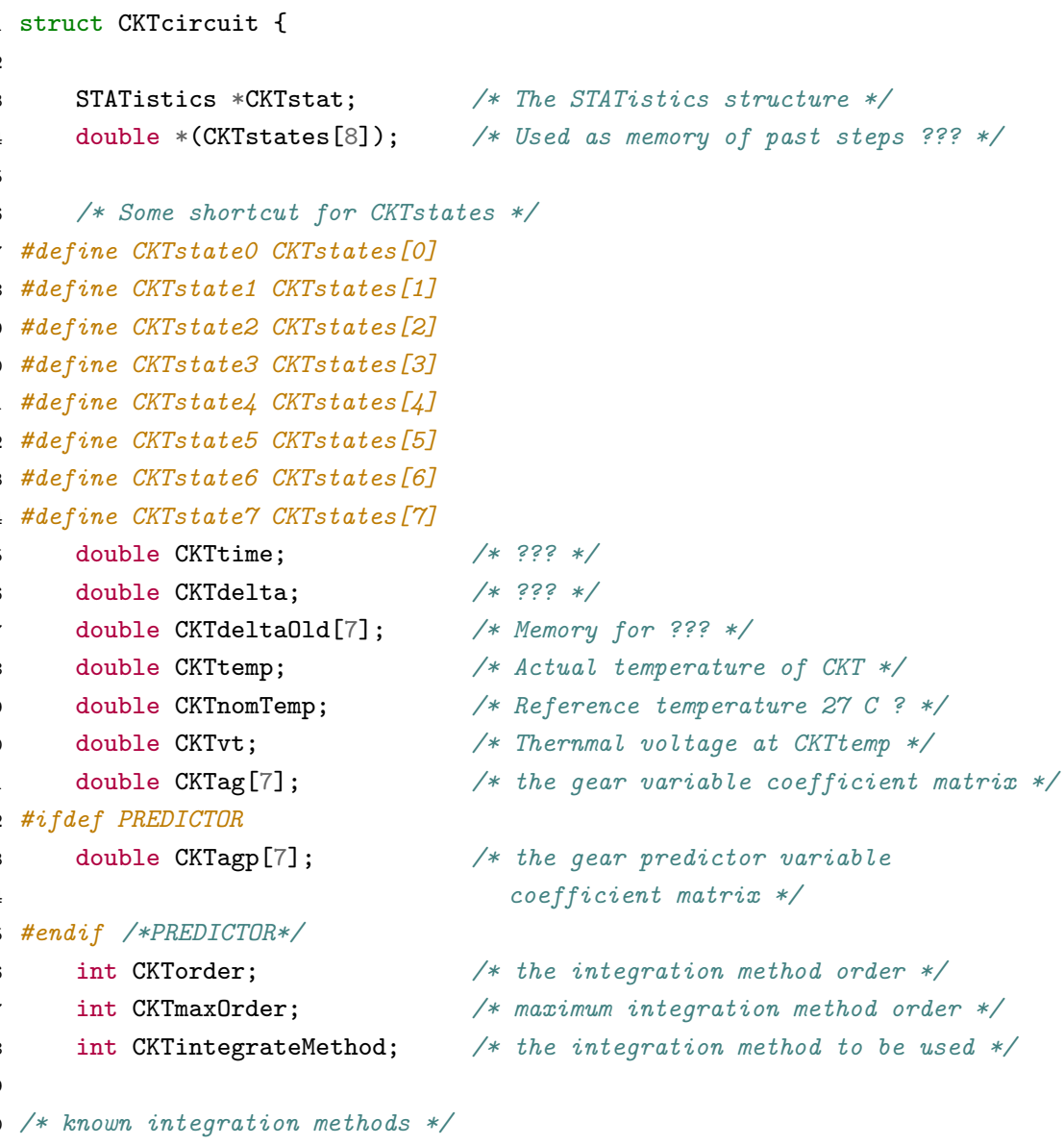




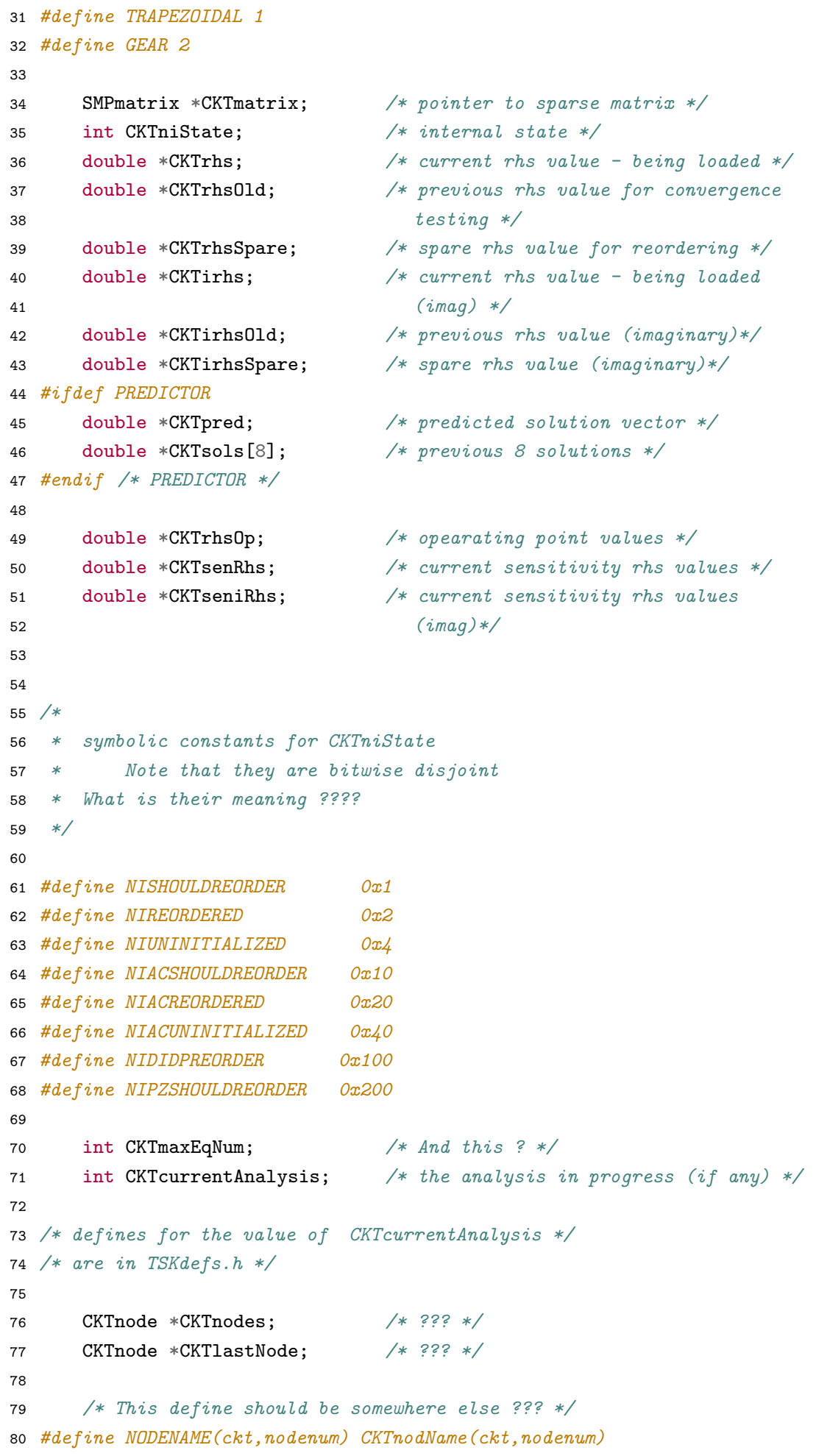




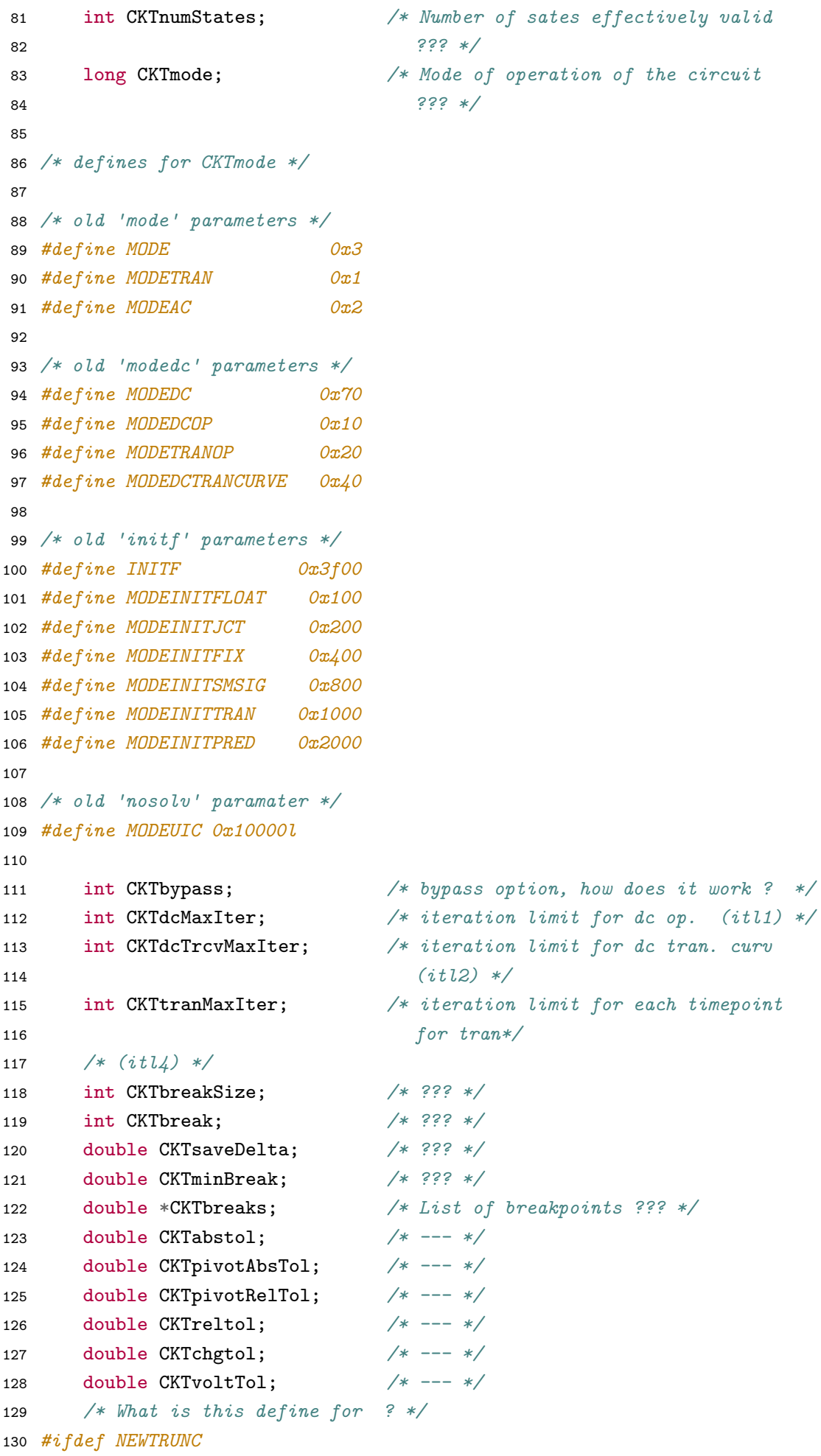




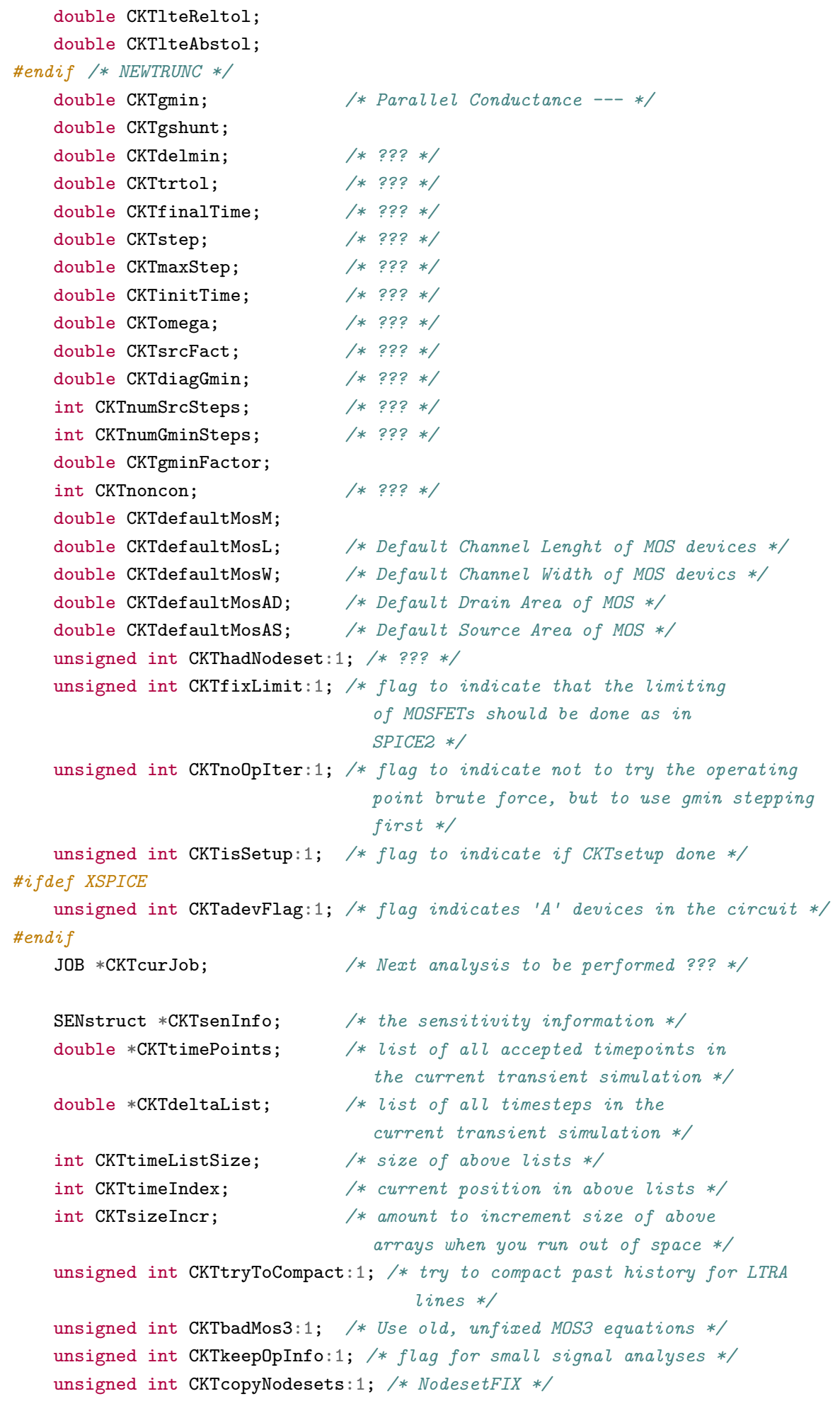




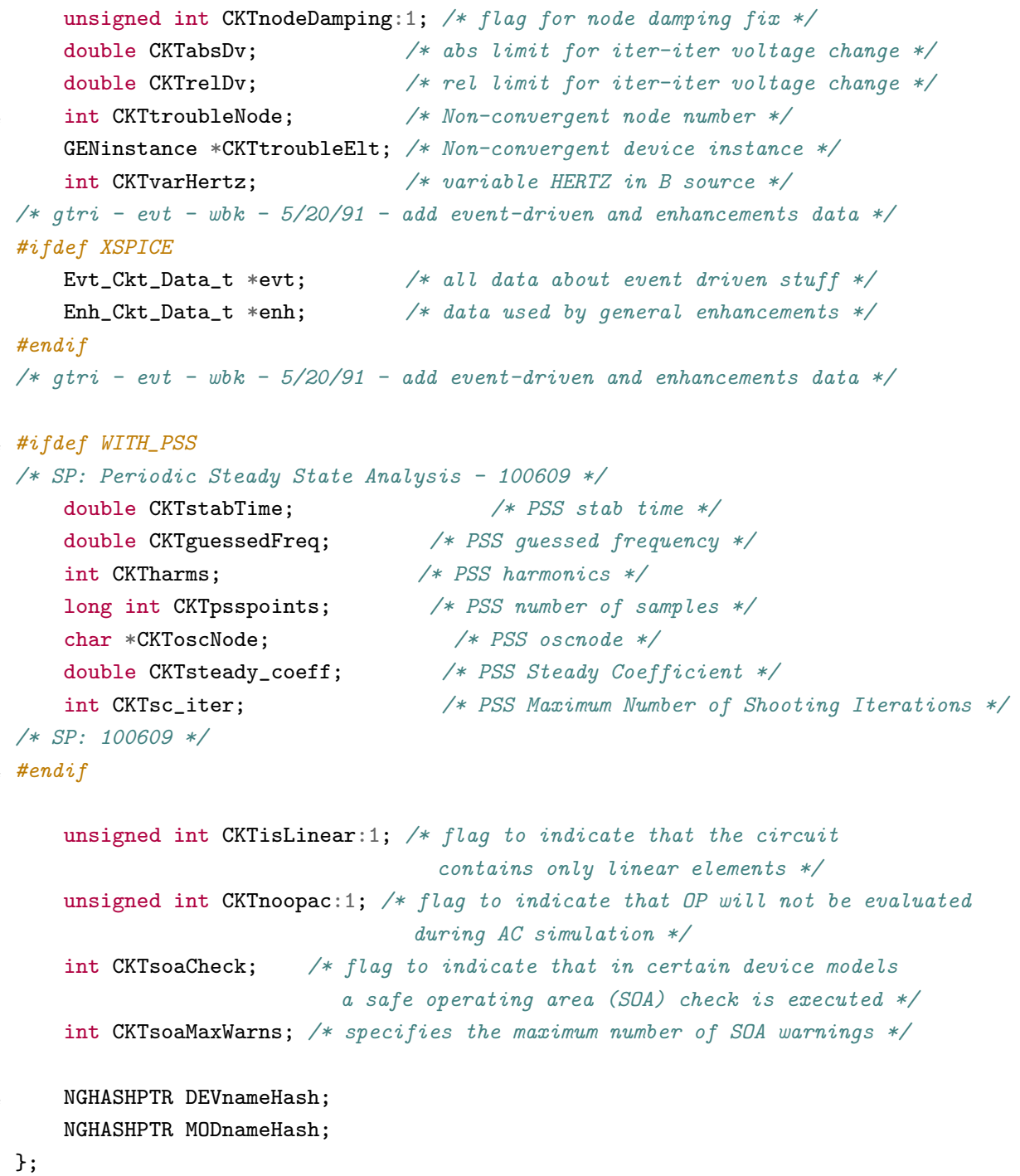




\section{Appendix B}

\section{Source code of the DIOload function}

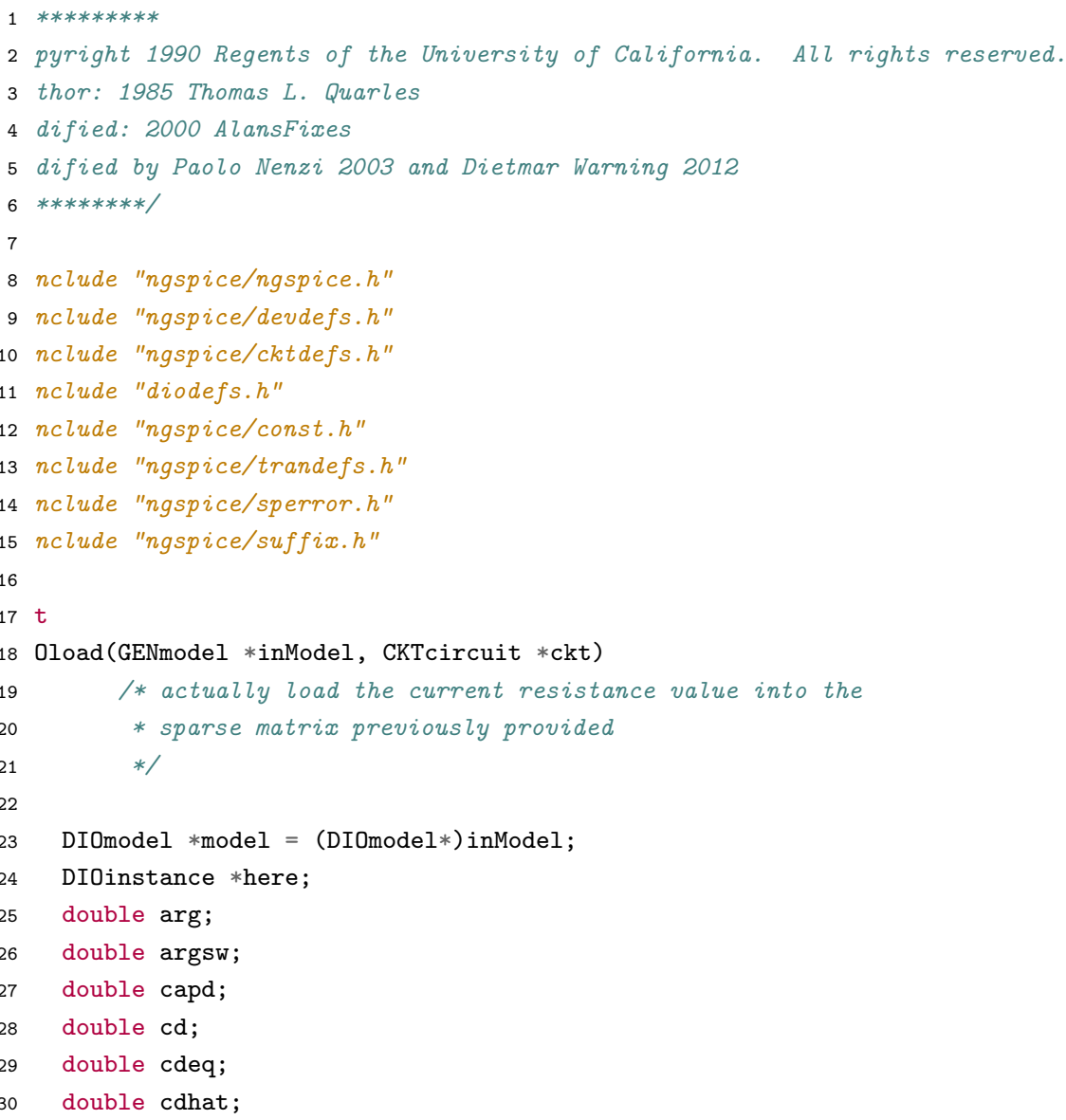




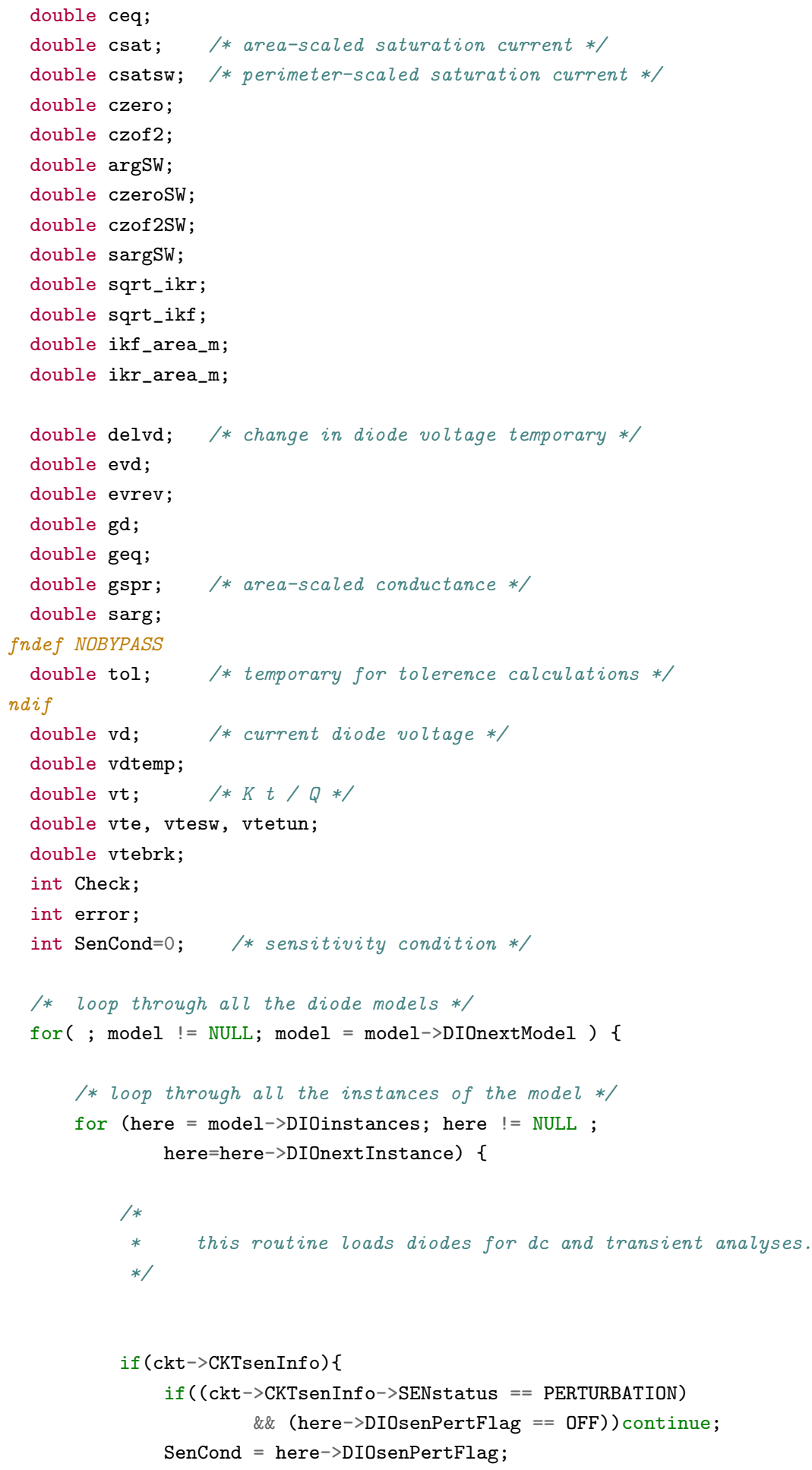




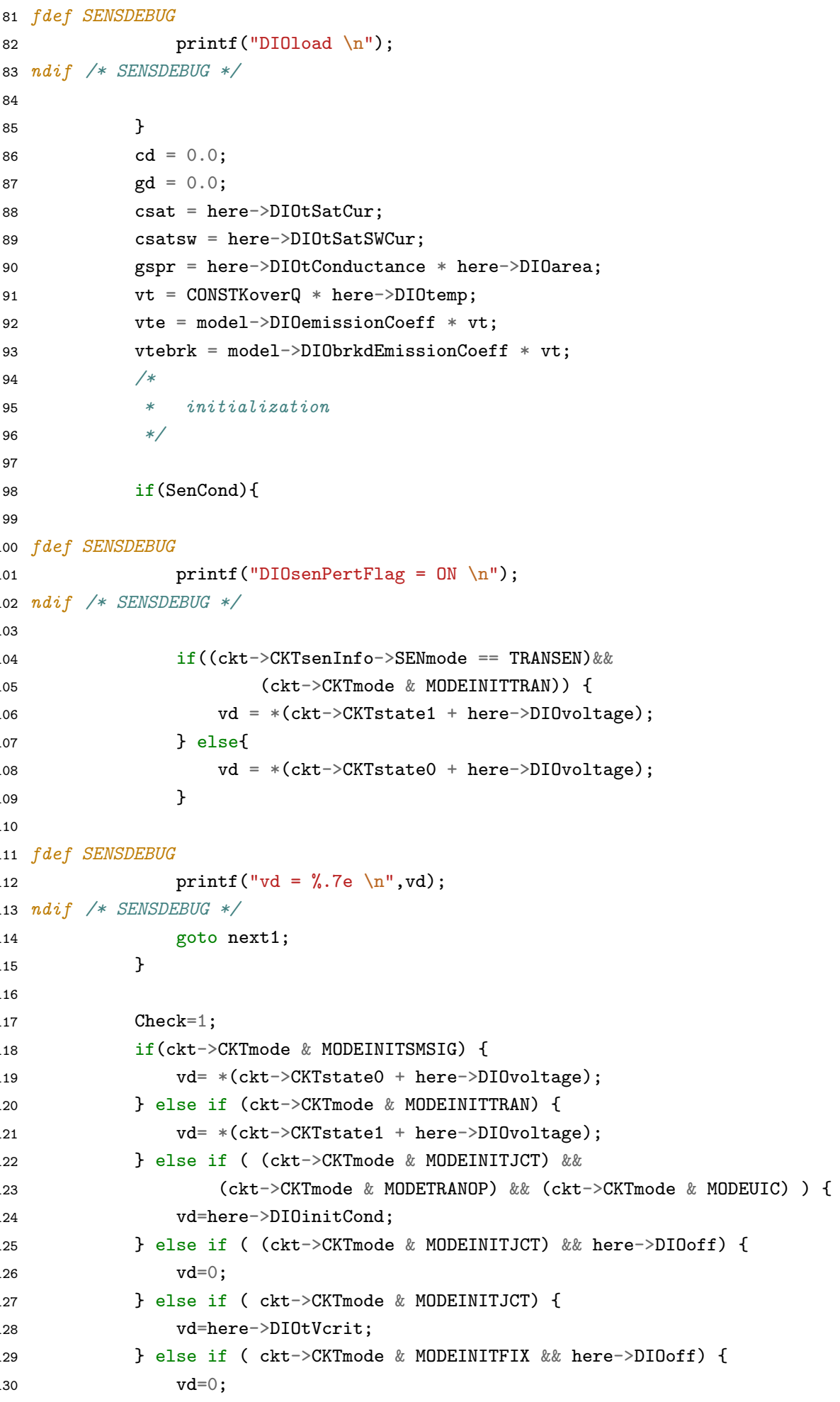




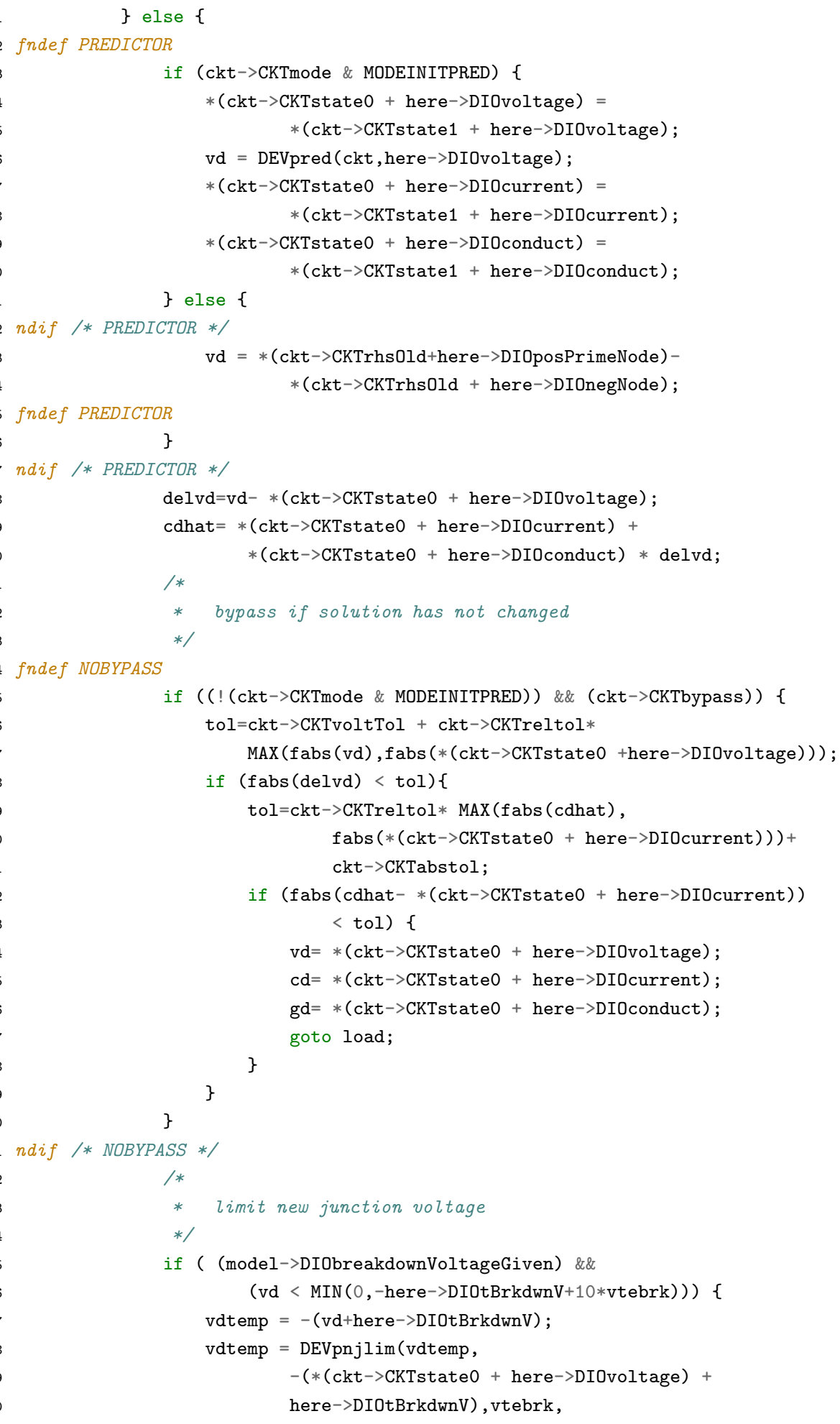




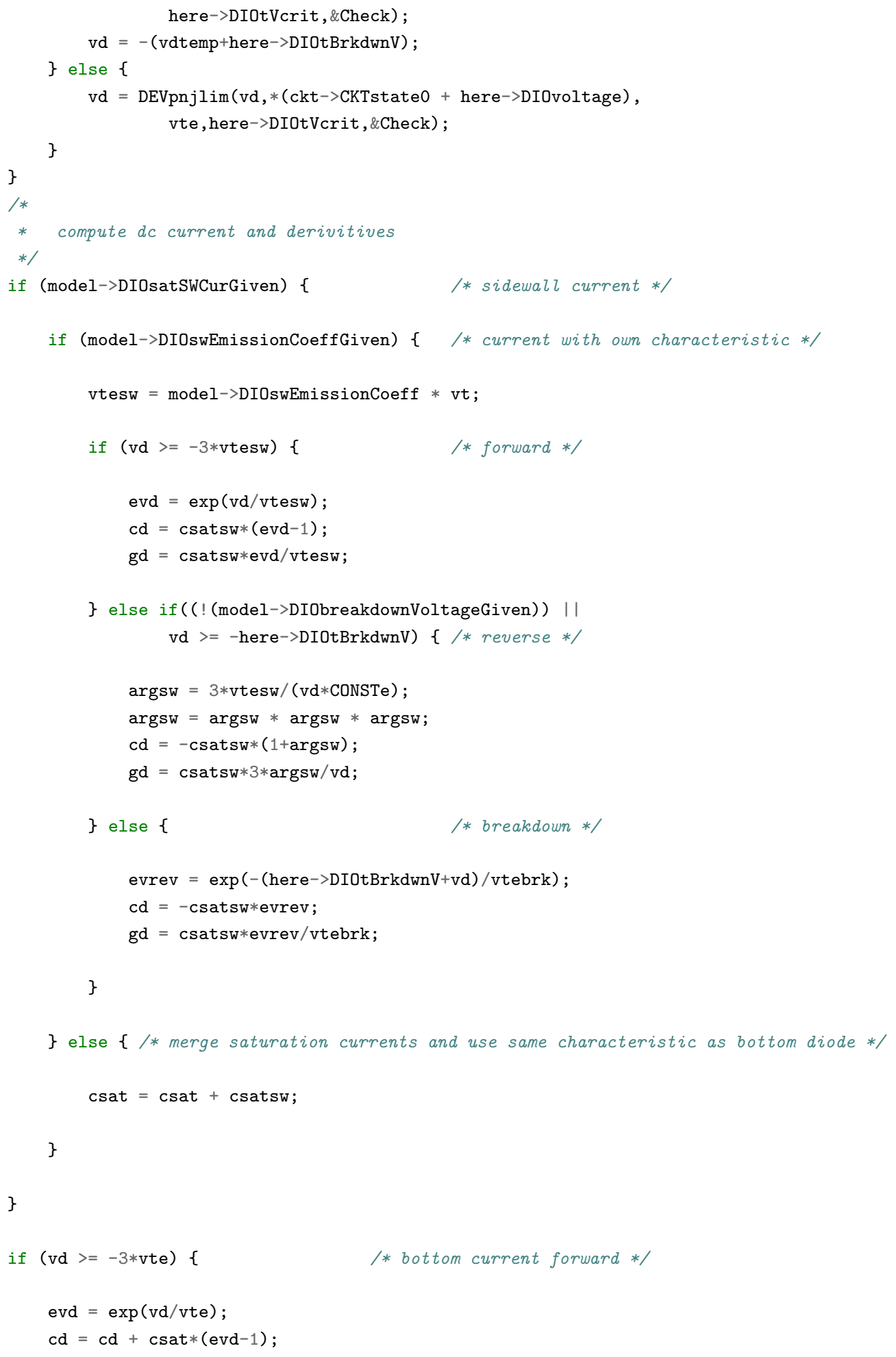




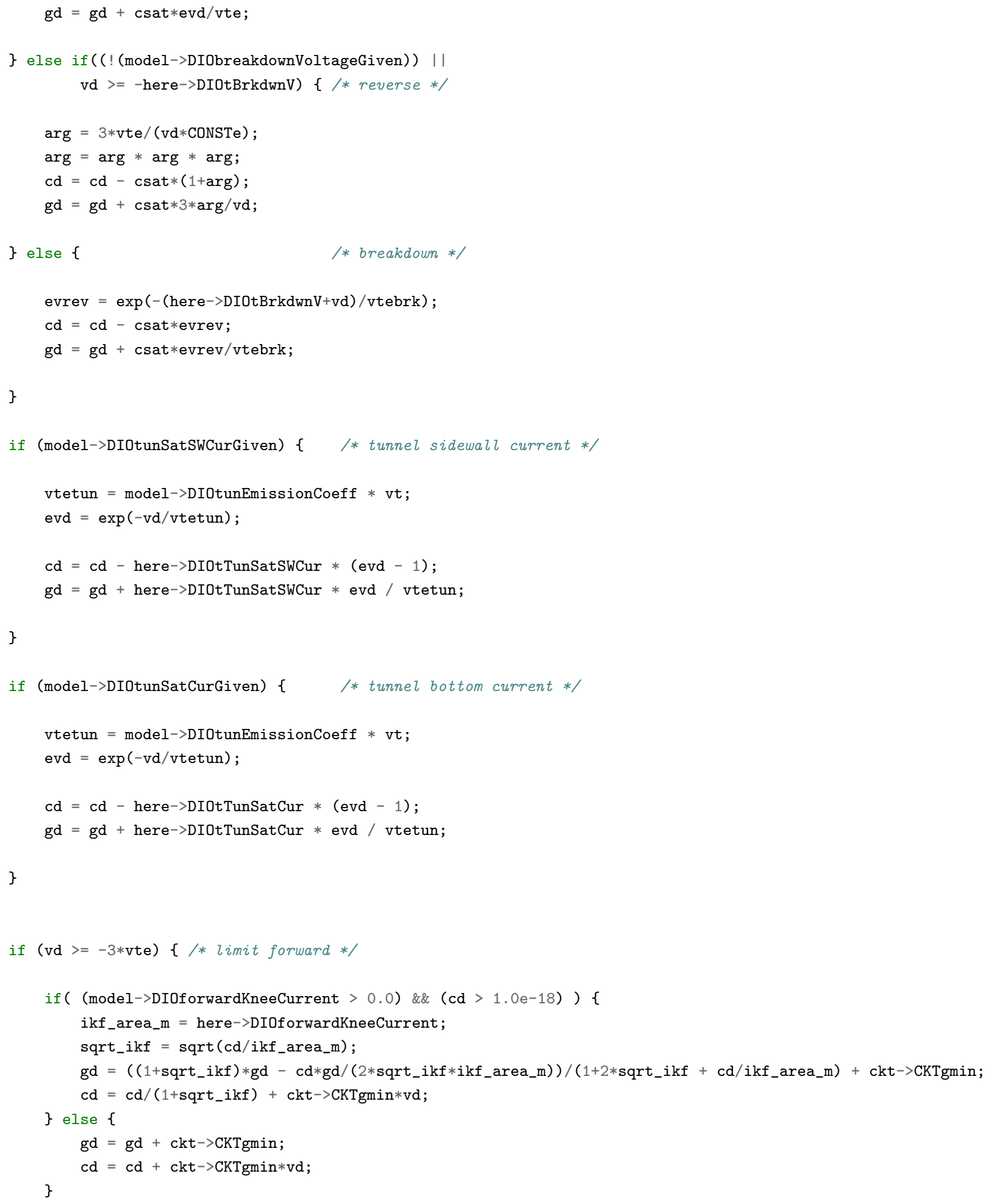




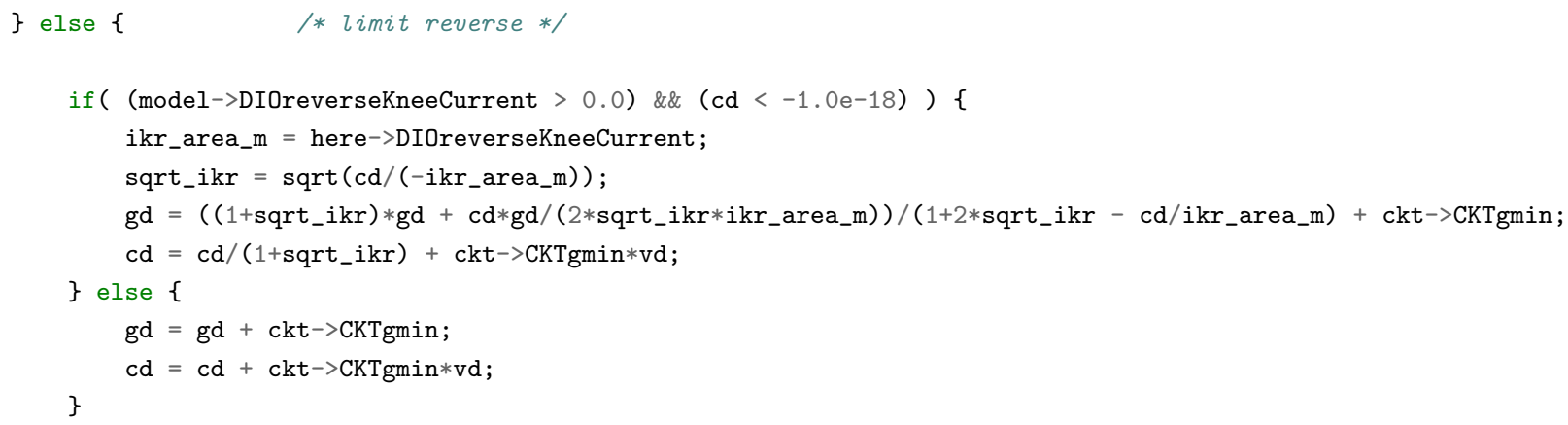


333

if (ckt->CKTmode \& MODEINITSMSIG) \{

*(ckt->CKTstate0 + here->DIOcapCurrent $)=$ capd;

if (SenCond) \{

$*($ ckt $->$ CKTstate $0+$ here $->$ DIOcurrent $)=\mathrm{cd}$;

*(ckt->CKTstate + here->DIOconduct $)=$ gd;

printf("storing small signal parameters $\backslash \mathrm{n} ")$; printf ("cd $=\% .7 \mathrm{e}, \mathrm{vd}=\% .7 \mathrm{e} \backslash \mathrm{n} ", \mathrm{~cd}, \mathrm{vd})$; 


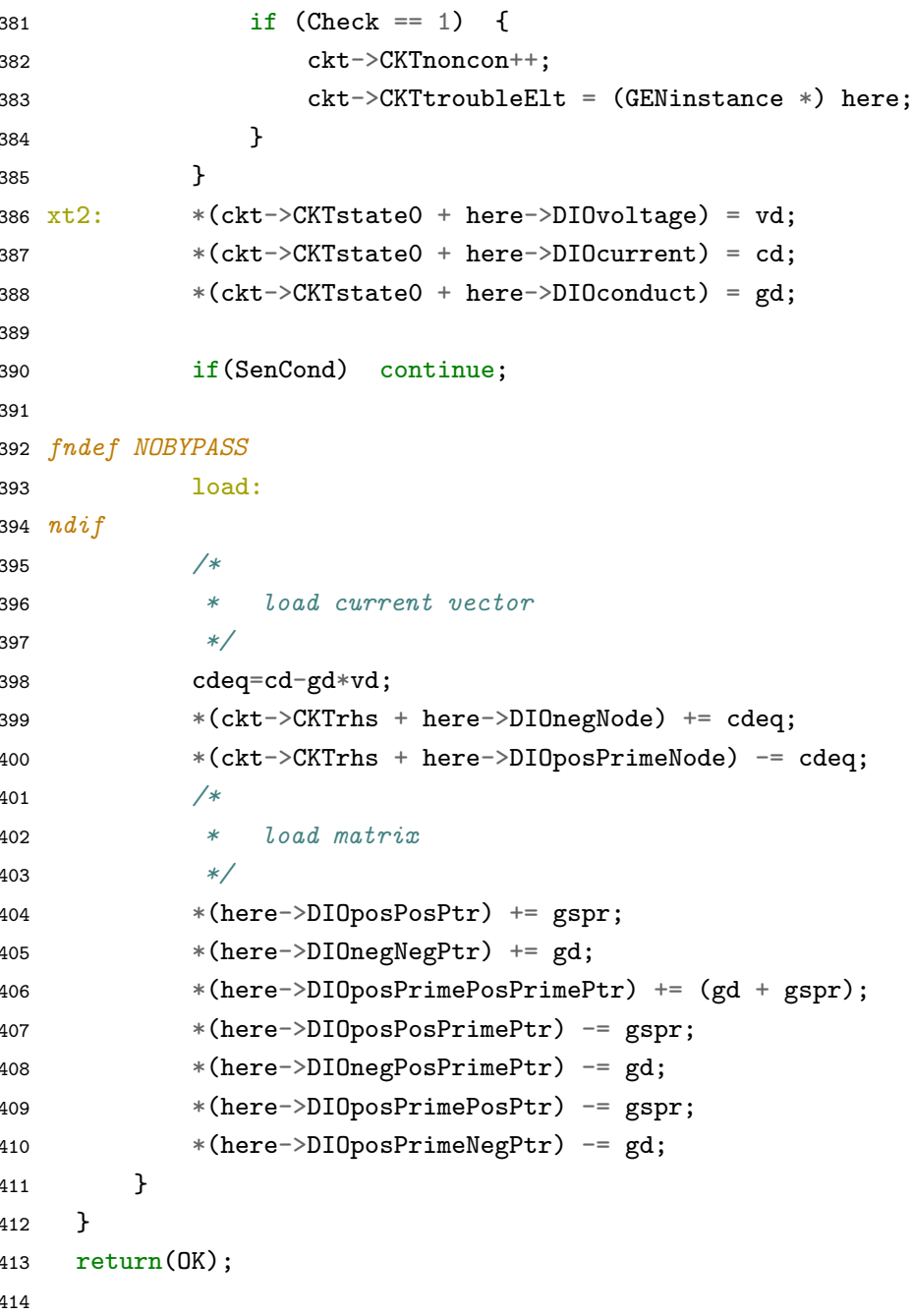




\section{References}

[1] K. Kundert, The Designer's Guide to SPICE and Spectre. Norwell, MA: Kluwer Academic Publishers, 1995.

[2] E. Gad, M. Nakhla, R. Achar, and Y. Zhou, "A-stable and L-stable highorder integration methods for solving stiff differential equations," IEEE Transactions on Computer-Aided Design of Integrated Circuits and Systems, vol. 28, no. 9, pp. 1359-1372, Sep. 2009.

[3] N. Obreshkov, "Sur les quadrature mecanique," (Bulgarian, French Summary) Akad. Nauk., vol. 65, pp. 191-289, 1942.

[4] G. Wanner, E. Hairer, and S. P. Nøsett, "Order stars and stability theorems," BIT, vol. 18, pp. 475-489, 1978.

[5] G. Birkoff and R. S. Varga, "Discretization errors for well-set cauchy problems," J. Math. and Physics, vol. 44, pp. 1-23, 1965. 
[6] B. L. Ehle, "High-order A-stable methods for the numerical solution of D.E.'S," BIT, vol. 8, pp. 276-278, 1968.

[7] Y. Zhou, E. Gad, M. S. Nakhla, and R. Achar, "Structural characterization and efficient implementation techniques for A-stable high-order integration methods," IEEE Transactions on Computer-Aided Design of Integrated Circuits and Systems, vol. 31, no. 1, pp. 101 -108, Jan. 2012.

[8] M. Farhan, E. Gad, M. Nakhla, and R. Achar, "New method for fast transient simulation of large linear circuits using high-order stable methods," IEEE Transactions on Components, Packaging and Manufacturing Technology, vol. 3, pp. 661-669, April 2013.

[9] — - "Fast simulation of microwave circuits with nonlinear terminations using high-order stable methods," IEEE Transactions on Microwave Theory and Techniques, vol. 61, pp. 360-371, 2013.

[10] G. A. Baker Jr., Essentials of Padé Approximants. New York: Academic Press, 1975.

[11] U. A. Ascher and L. R. Petzold, Computer Methods for Ordinary Differential Equations and Differential-Algebraic Equations. SIAM, 1998. 
[12] C. Gear, "Simultaneous numerical solution of differential-algebraic equations," IEEE Transactions on Circuit Theory, vol. 18, no. 1, pp. 89-95, Jan. 1971.

[13] J. D. Lambert, Computational Methods in Ordinary Differential Equations, ser. Introductory mathematics for scientists and engineers. London Wiley, 1973.

[14] J. C. Butcher, Numerical Methods for Ordinary Differential Equations. Wiley, 2003.

[15] C.-W. Ho, A. Ruehli, and P. Brennan, "The modified nodal approach to network analysis," Circuits and Systems, IEEE Transactions on, vol. 22, no. 6, pp. $504-509$, June 1975 .

[16] L. W. Nagel, "Spice2: A computer program to simulate semiconductor circuits," Ph.D. dissertation, EECS Department, University of California, Berkeley, 1975.

[17] A. S. Sedra and K. C. Smith, Microelectronic Circuits. New York: OXFORD, 2004.

[18] J. Vlach and K. Singhal, Computer Methods for Circuit Analysis and Design. New York: Van Nostrand Reinhold, 1983. 
[19] A. Griewank, Evaluating Derivatives: Principles and Applications of Algorithmic Differentiation. SIAM, 2000.

[20] J. R. Gilbert and T. Peierls, "Sparse partial pivoting in time proportional to arithmetic operations," SIAM Journal on Scientific and Statistical Computing, vol. 9, no. 5, pp. 862-874, 1988. [Online]. Available: http://link.aip.org/link/?SCE/9/862/1

[21] T. A. Davis, E. P. Natarajan, and A. Inc, "Algorithm 907: Klu, a direct sparse solver for circuit simulation problems," ACM Transactions on Mathematical Software.

[22] R. Sedgewick and K. Wayne, Algorithms. Pearson Education, 2011.

[23] T. L. Quarles, "Analysis of performance and convergence issues for circuit simulation," Ph.D. dissertation, EECS Department, University of California, Berkeley, 1989.

[24] Y. Lin and E. Gad, "Formation of the obreshkov based transient circuit simulator in the presence of nonlinear memory elements," IEEE Transactions on Computer-Aided Design of Integrated Circuits and Systems, vol. 34, no. 1, pp. 86-94, 2015. 
[25] B. C. Lopes and R. Auler, Getting Started with LLVM Core Libraries. Packt Publishing, 2014. 